\title{
Metrology and Data for Microelectronic Packaging and Interconnection
}

\section{NTSUI: 3521}

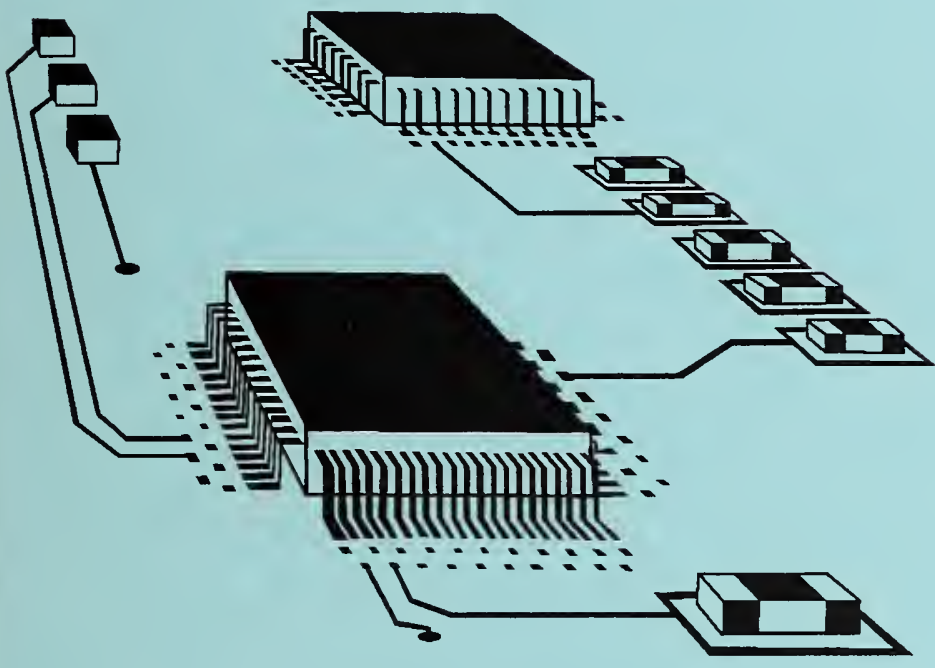

Results of a joint workshop on Materials Metrology and Data for Commercial Electrical and Optical Packaging and Interconnection Technologies

May 5-6, 1994, Gaithersburg, MD.

Edited by Michael A. Schen

National Institute of Standards and Technology

Sponsored by:

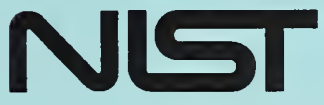

U.S. DEPARTMENT OF COMMERCE Technology Administration National Institute of Standards and Technology

Institute for Interconnecting and

Packaging Electronic Circuits

Optoelectronics Industry Development Association

Semiconductor Research Corporation

QC

100

.056

1.0. 5520

1994

V.2 



\section{Metrology and Data for Microelectronic Packaging and Interconnection}

\section{Results of a joint workshop on Materials Metrology and Data for Commercial Electrical and Optical Packaging and Interconnection Technologies}

Edited by Michael A. Schen

National Institute of Standards and Technology

Cosponsored by NIST, the Institute for Interconnecting and Packaging Electronic Circuits, the Optoelectronics Industry Development Association, and the Semiconductor Research Corporation.

November 1994

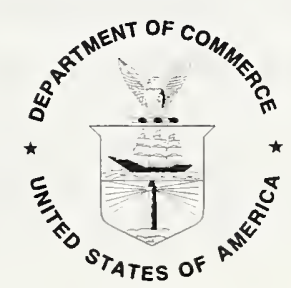

U.S. DEPARTMENT OF COMMERCE Ronald H. Brown, Secretary

TECHNOLOGY ADMINISTRATION

Mary L. Good, Under Secretary for Technology

NATIONAL INSTITUTE OF STANDARDS AND TECHNOLOGY Arati Prabhakar, Director 

COVER ILLUSTRATION

Schematic illustration of contemporary single-chip electronic packaging and interconnection technology consisting of surface mounted components, quad flat-pack semiconductor package, plated through holes and fine-pitch interconnection.

\section{DISCLAIMER}

Commercial equipment, instruments, software, materials or services are identified to adequately report the discussions that took place. Such identification does not constitute nor imply recommendation, endorsement, or criticism by the National Institute of Standards and Technology, the Institute for Interconnecting and Packaging Electronic Circuits, the Optoelectronics Industry Development Association, or the Semiconductor Research Corporation. 



\section{TABLE OF CONTENTS}

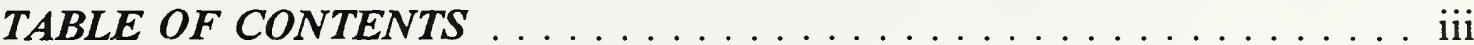

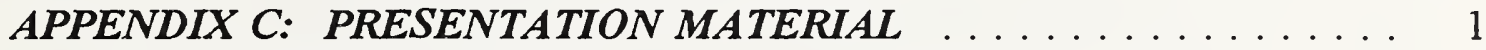

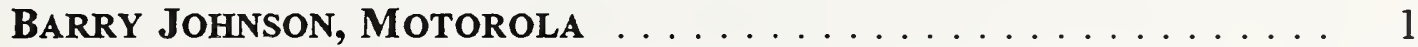

PaUl haugsiaA, GTE Laboratories $\ldots \ldots \ldots \ldots \ldots \ldots$

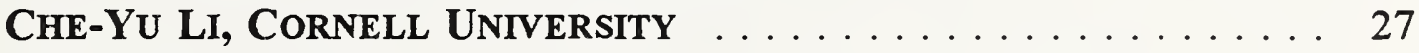

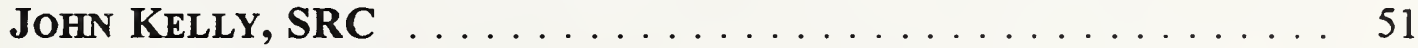

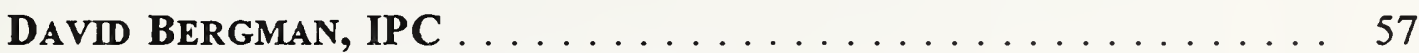

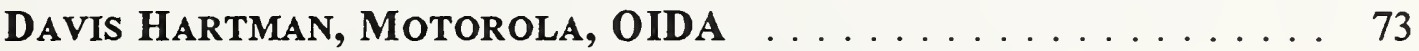

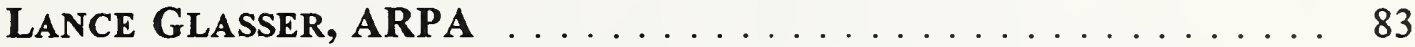

MARSHALL ANDREWS, ITRI $\ldots \ldots \ldots \ldots \ldots \ldots \ldots \ldots$

MICHAEL SCHEN, NIST . . . . . . . . . . . . . . . . . 119

JOHN GUDAS, NIST . . . . . . . . . . . . . . . . . . 123

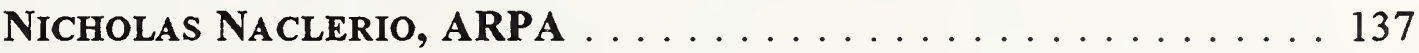





\section{APPENDIX C: PRESENTATION MATERIAL}

\section{BARRY JOHNSON, MOTOROLA}


(Mi) MOTOROLA

Cristrionos

\title{
MATERIALS CHALLENGES IN MICROELECTRONIC PACKAGING AND INTERCONNECTION
}

\author{
Barry Johnson \\ Motorola \\ Tempe, Arizona
}

May 5, 1994

(M) MOTOROLA

\section{OUTLINE}

1. MARKET TRENDS

- SEGMENTS/DRIVING FORCES

- MPU EVOLUTION

2. IMPACT ON PACKAGING

- PERFORMANCE ISSUES

- DESIGN / SIMULATION

3. MULTI-CHIP MODULES

- SYSTEM'S APPROACH

- DESIGN GUIDELINES

4. PACKAGING TRENDS 
(M) MOTOROLA

\section{MARKET SEGMENTS / DRIVING FORCES}

\section{SEGMENT}

\section{Computer}

Mainframe/Supercomputer

Workstation/PC

Communications

Fixed

Mobile

Space

Automotive

Body

Powertrain

Electric Vehicle

Industrial

Instrumentation

Power Control

Consumer

Fixed

Portable/Hand-Held

\section{DRIVING FORCE}

Performance

Performance/Cost

Cost

Size, Cost

Performance, Size, Weight

Cost, Size, Weight

Performance, Cost, Size, Weight

Performance, Cost, Weight

Performance, Cost

Performance

Cost

Cost. Size, Weight

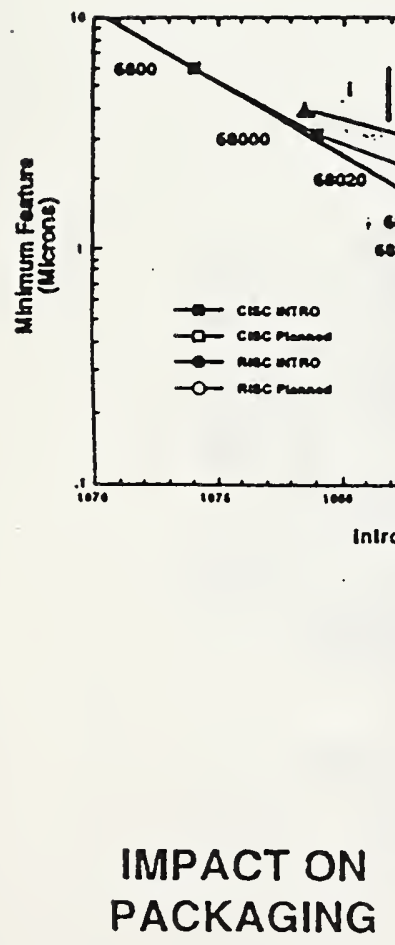
MICROPROCESSOR
CIRCUIT TRENDS

PACKAGING

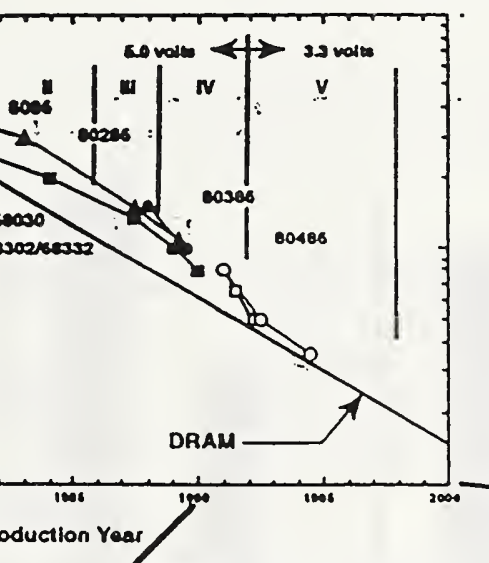



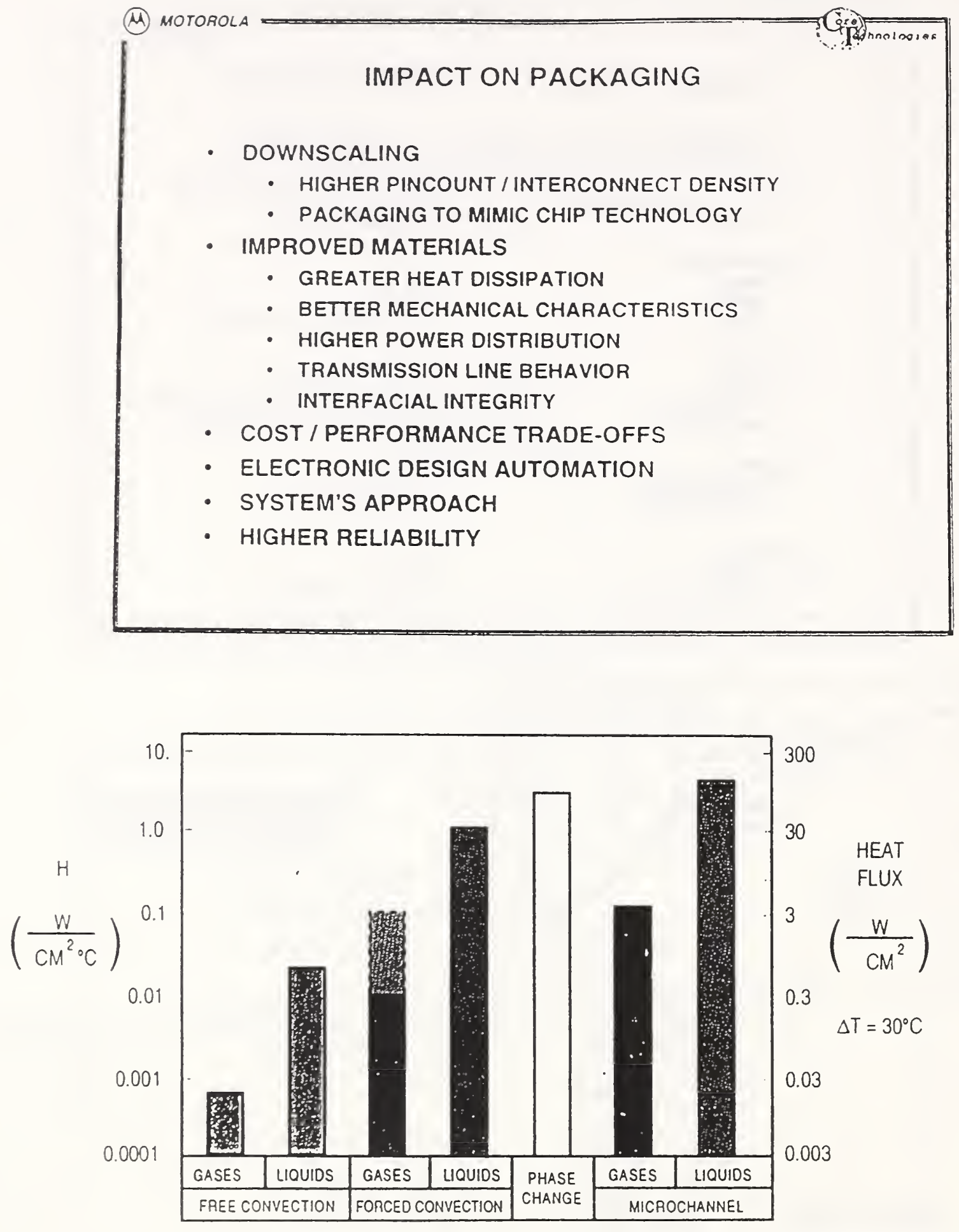

REF: MAHALINGAM (MOD.) 

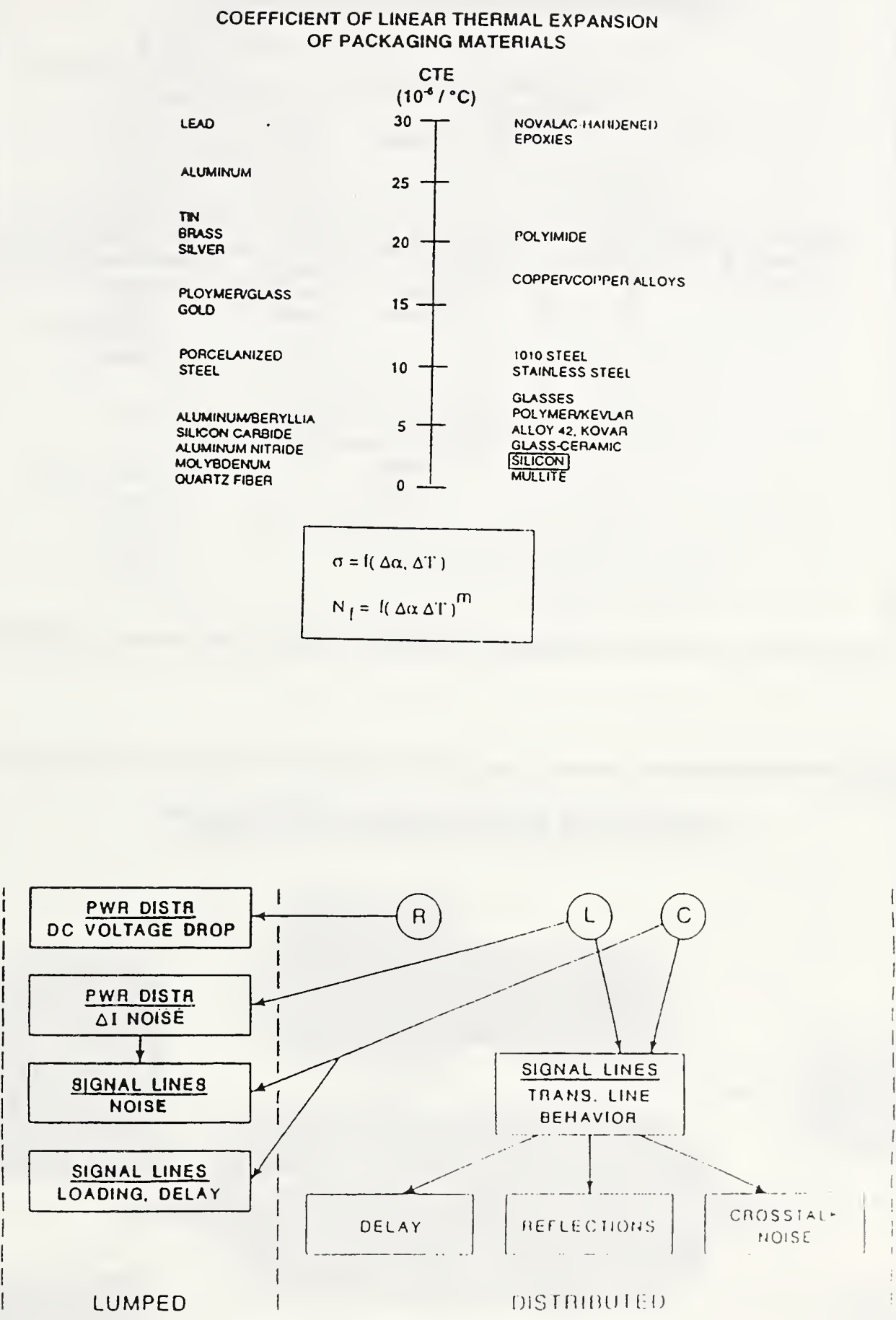


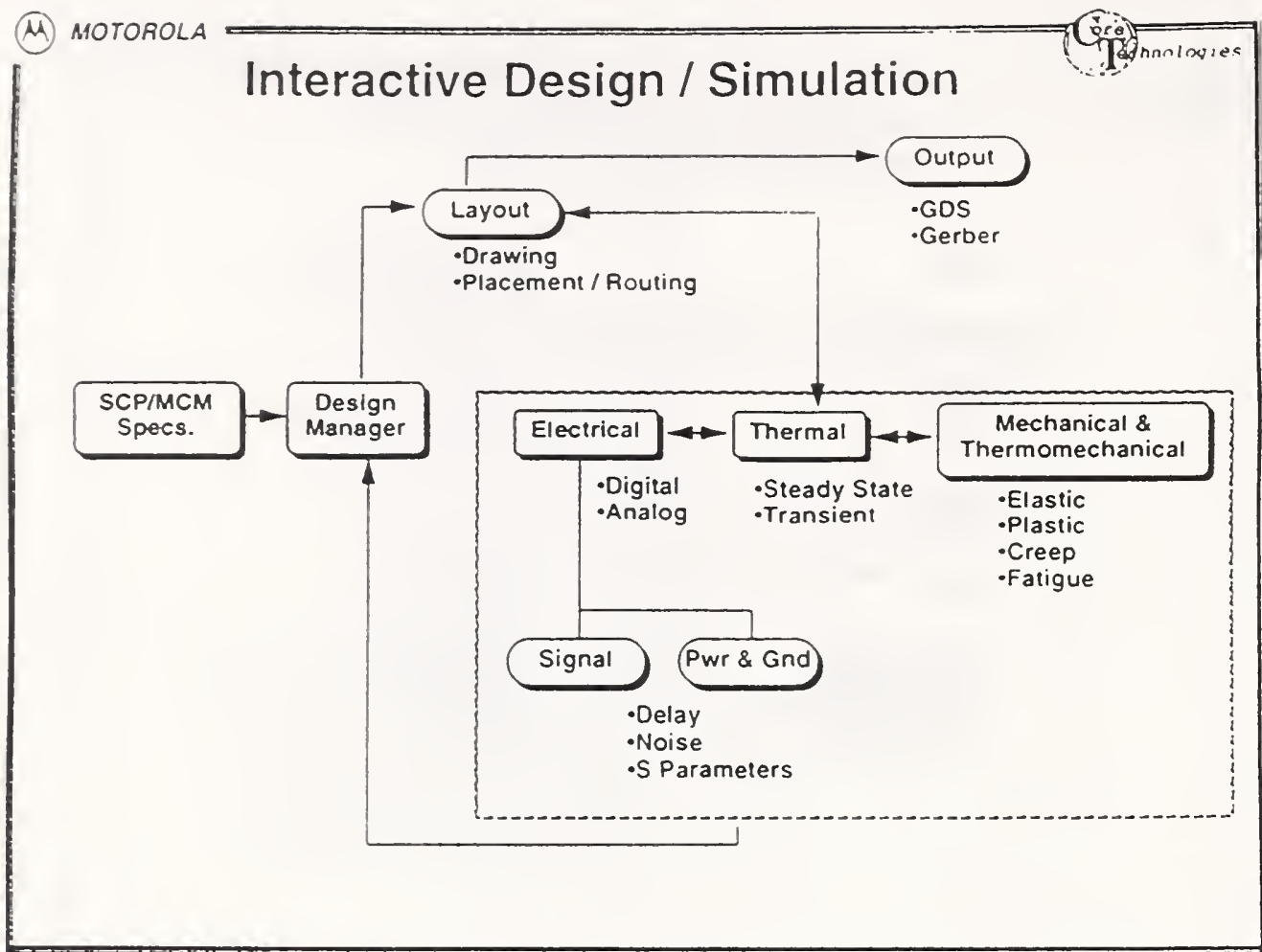

(44)

Interfaces In Conventional Packages

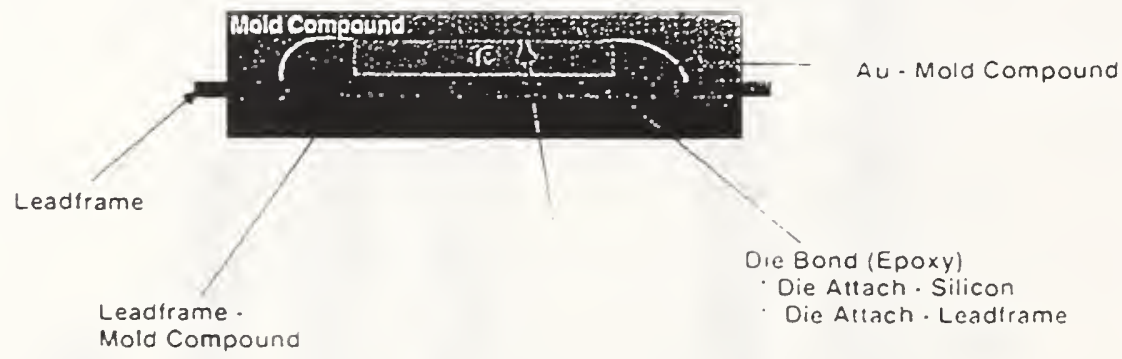

Chip Hold Compound

apdo 


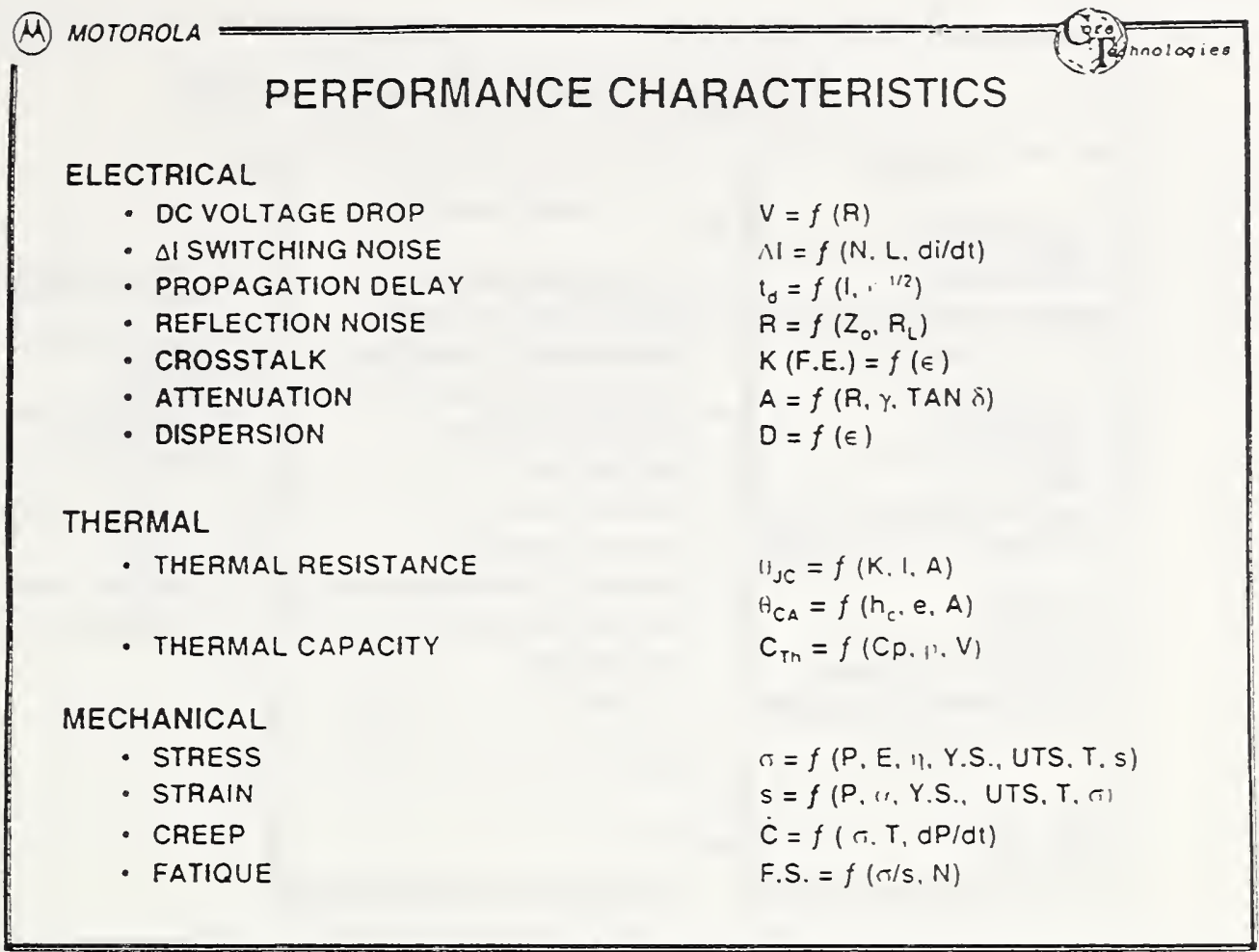

\section{MOLDED PLASTIC PACKAGE}

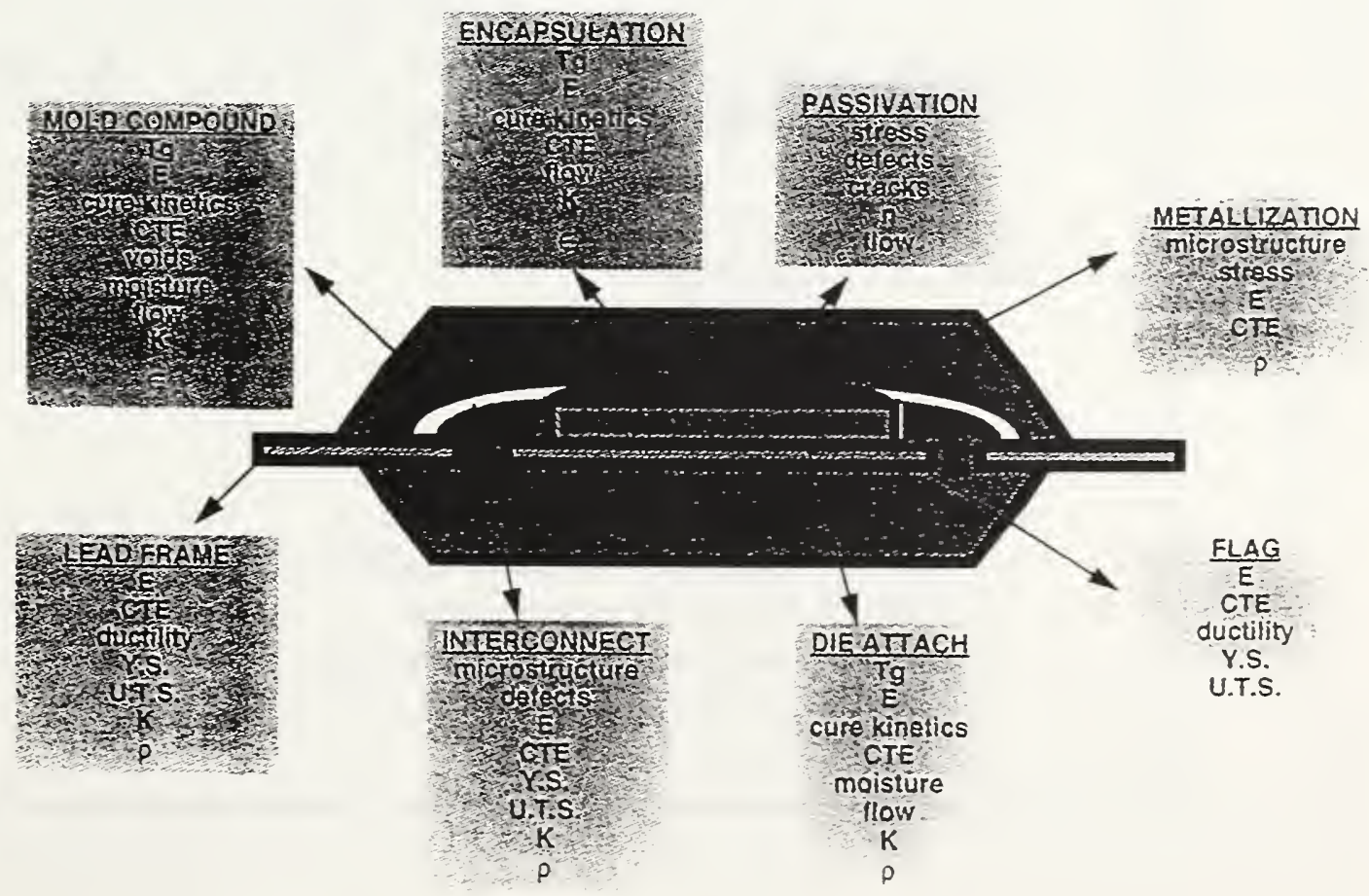




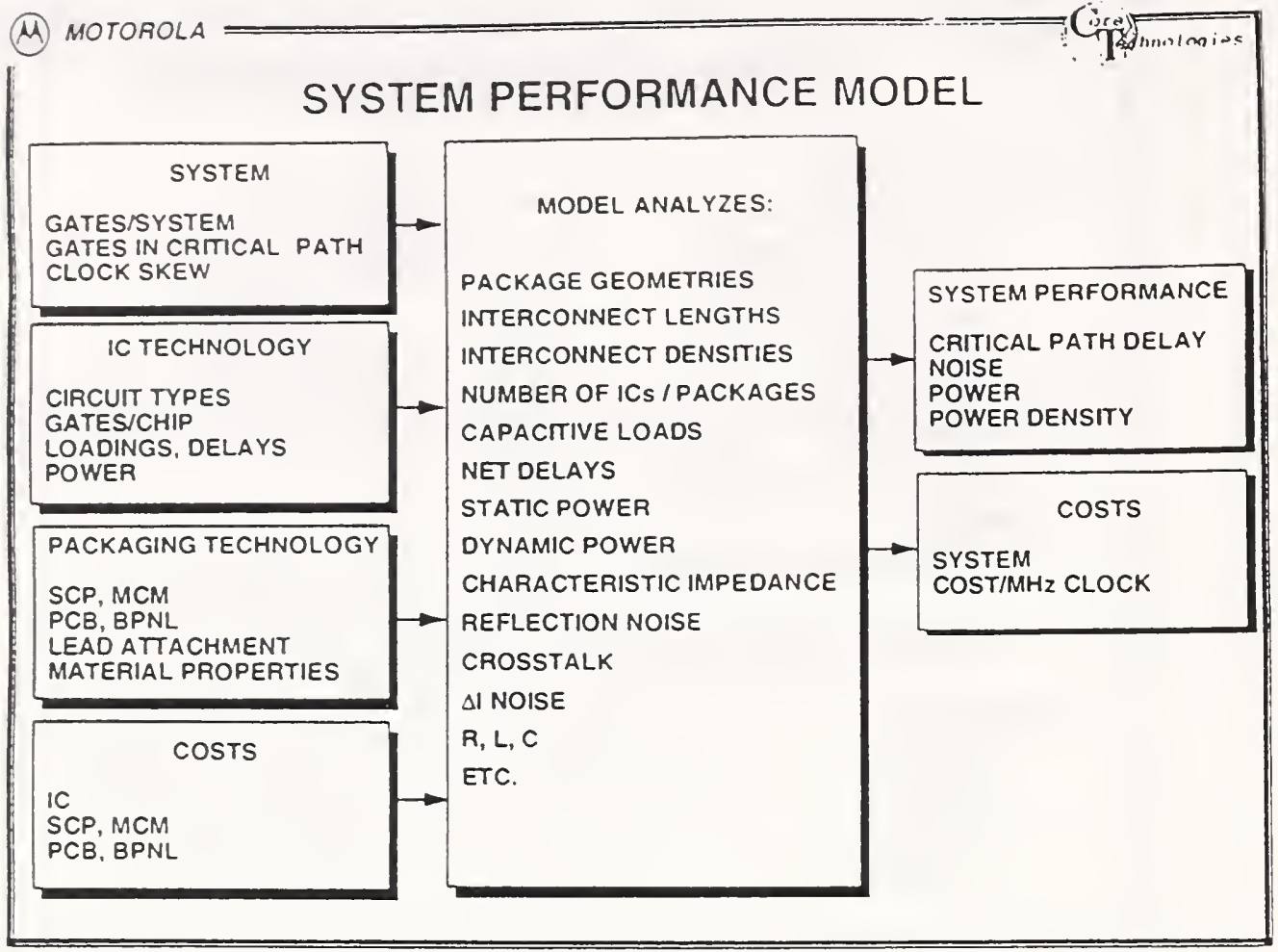

(M) MOTOROLA

\section{SYSTEM PERFORMANCE ENHANCEMENTS}

- ARCHITECTURE

$10-100 x$

- parallel pipelined haRdWARE

- SOFTwARE

- BASIC TECHNOLOGIES

$2-10 x$

- LEVEL OF INTEGRATION

- IC gate DELAYIDRIVE CAPABILITY

- PACKAGING DENSITY

- IMPROVEMENTS WITHIN TECHNOLOGY

$2-5 x$

- chip/PACKaGe MATERIALs

- number of chips per package

- REDUCED PROPAGation DELAy/NOISE 


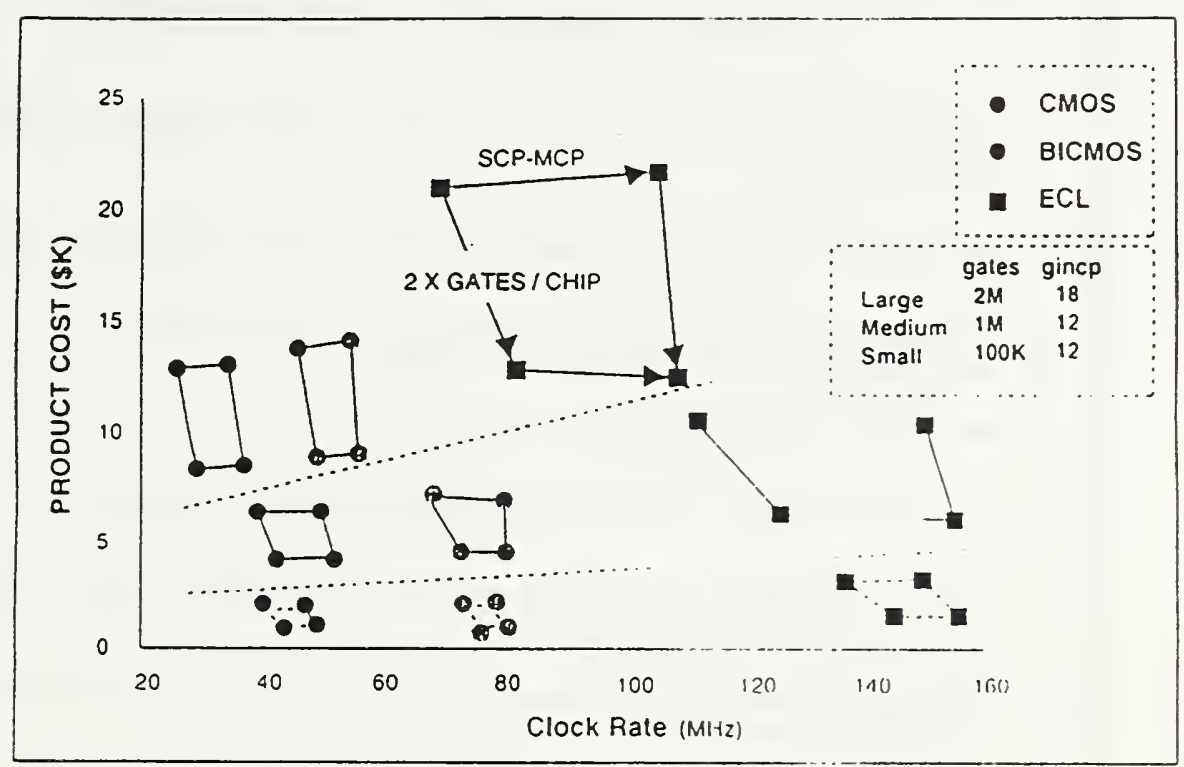

\section{CIRCUIT AND PACKAGING COSTS VERSUS CLOCK RATE}

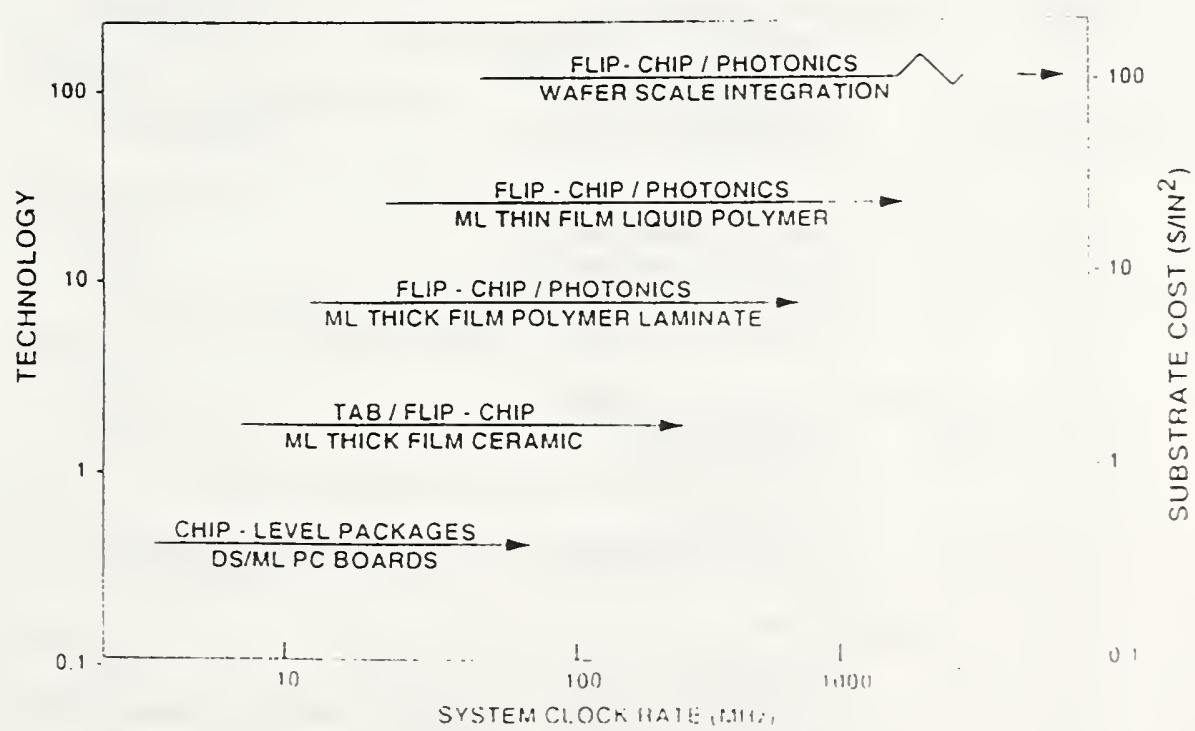


(M) MOTOROLA

\section{DESIGN GUIDELINES FOR HIGH PERFORMANCE PACKAGING}

1. SPACE INTEGRATED CIRCUITS AS CLOSELY AS HEAT REMOVAL TECHNIQUES ALLOW.

2. PARTITION HEAT REMOVAL AND ELECTRICAL INTERCONNECT PATHS.

3. INTERCONNECT CIRCUITS WITH HIGH ASPECT RATIO SIGNAL LINES PATTERNED IN HIGH CONDUCTIVITY METAL.

MAXIMIZE CROSS-SECTION OF POWER LINES.

4. SEPARATE SIGNAL LINES AS FAR APART AS WIRING LAYERS ALLOW.

5. SEPARATE SIGNAL LINES FROM GROUND PLANES WITH THICK LAYER OF L'OW K DIELECTRIC MATERIAL.

SEPARATE POWER AND GROUND LINES/PLANES WITH THIN LAYER OF HIGH K DIELECTRIC MATERIAL.

6. DESIGN IN TESTABILITY AND REPAIRABILITY AT ALL STAGES OF ASSEMBLY.

(M) MOTOROLA

MULTI LAYER MCM SUBSTRATES

INTERCONNECTS

- LOW ELECTRICAL RESISTIVITY $(C u=1.7 \mathrm{MICRO}$ OHM.cm)

- FINE LINE PATTERnABILITY

- LOW STRESS

- ADHESION

- LOW DIFFUSION KINETICS

SURFACE LAYERS

- LOW CTE $(\mathrm{Si}=2.6 \mathrm{PPM} / \mathrm{C})$

- DIELECTIC CONSTANT $<6$

- DIMENSIONAL STABILITY, $<0.2 \%$

- provide Reliable vias and plated-through.holes

- WTHSTAND ASSEMBLY TEMPERATURES (UP TO 600C)

INTERLEVEL DIELECTRICS

- noncorrosive

- GOOD ADHESION

- Low film StRess and high crack resistance

PLANARIZING

- dielectric constant < 3 for signal distribution

- DIELECTRIC CONSTANT $>3$ FOR POWER DISTRIBUTION

PASSIVATION

- dimensional STABILITY

- low stRess and high crack resistance

- adHESION TO MULTIPLE MATERIALS - METALS OXIDES. NITRIDES ETO

CHEMICALLY INACTIVE INTERFACES

- moisture and heat resistance

- HIGH THERMAL CONDUCTIVITY 
(4)

MOTOROLA

[egthnolagias

\section{INTERCONNECT EVOLUTION}

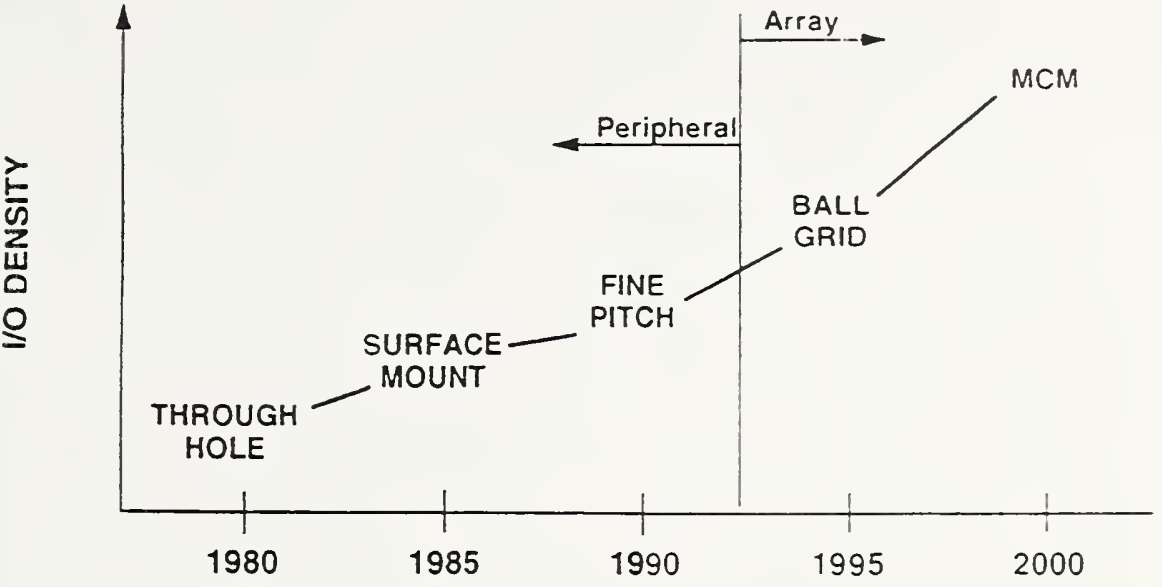

(4) MOTOROLA

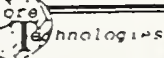

MARKET SEGMENTS / PACKAGING TRENDS

SEGMENT

Computer

Mainframe/Supercomputer

Workstation/PC

Communications

Fixed

Mobile

Space

Automotive

Body

Powertrain

Electric Vehicle

Industrial

Instrumentation

Power Control

Consumer

Fixed

Portable/Hand-Held

\section{CURRENT}

Array TH/SM, MCM

Peripheral SM

Peripheral TH/SM

Array SM

TH/SM

TH/SM

Peripheral TH/SM

Peripheral TH/SM

Power MCM

Peripheral SM

Peripheral TH/SM
Peripheral SM

Hi Power M.1CM

Array SM, MCM Array SM. MCM

Array SM

Array SM. MCM

MCM

$S M$

SM, MCM

Hi Power MCM

SM. $\mathrm{M} . \mathrm{CN}^{\mathrm{N}}$ 


\section{Paul haugsjaa, GTe laboratories}




\section{Emerging Applications for Low-Cost Optoelectronics}

\section{Paul Haugsjaa}

GTE Laboratories Incorporated Waltham, Massachusetts

May 5, 1994

\section{Scope of Presentation:}

-What is optoelectronics?

- Drivers for technology today

- Important cultural forces

$\diamond$ Evolving technological needs

- Growth areas of OE utilization

$\checkmark$ Associated challenges to be met 


\section{Optoelectronics:}

The technology of the interface between the realms of electronics and of light

\section{Technology Drivers}

- Grand problems

- Cancer, immune system, genetics

- Weather

- Unified physical theory

- World economy, etc.

- Improvement in life standards

- More of the same - world-wide - means costs must be reduced

- Advances dictate strong cost reductions 


\section{Evolving lifestyles - a search for:}

- Greater productivity

$\Delta$ Free time

- Instant information /entertainment

$\diamond$ Ubiquitous communications

- Low-cost, safe, reliable transportation

- Better medical care

- Improved personal security

\section{Evolving Technological Needs}

- Personal Communications System (PCS)

- Seamless information network

- Video transport to personal nodes

- Increased computer processing power

- Large, portable, high resolution displays with touch screens

- Low-cost, portable, high resolution printers

- Computer assisted transportation - control, collision avoidance, positioning, routing 


\section{Evolving Network Topology}

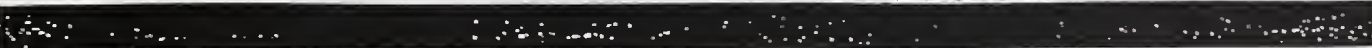

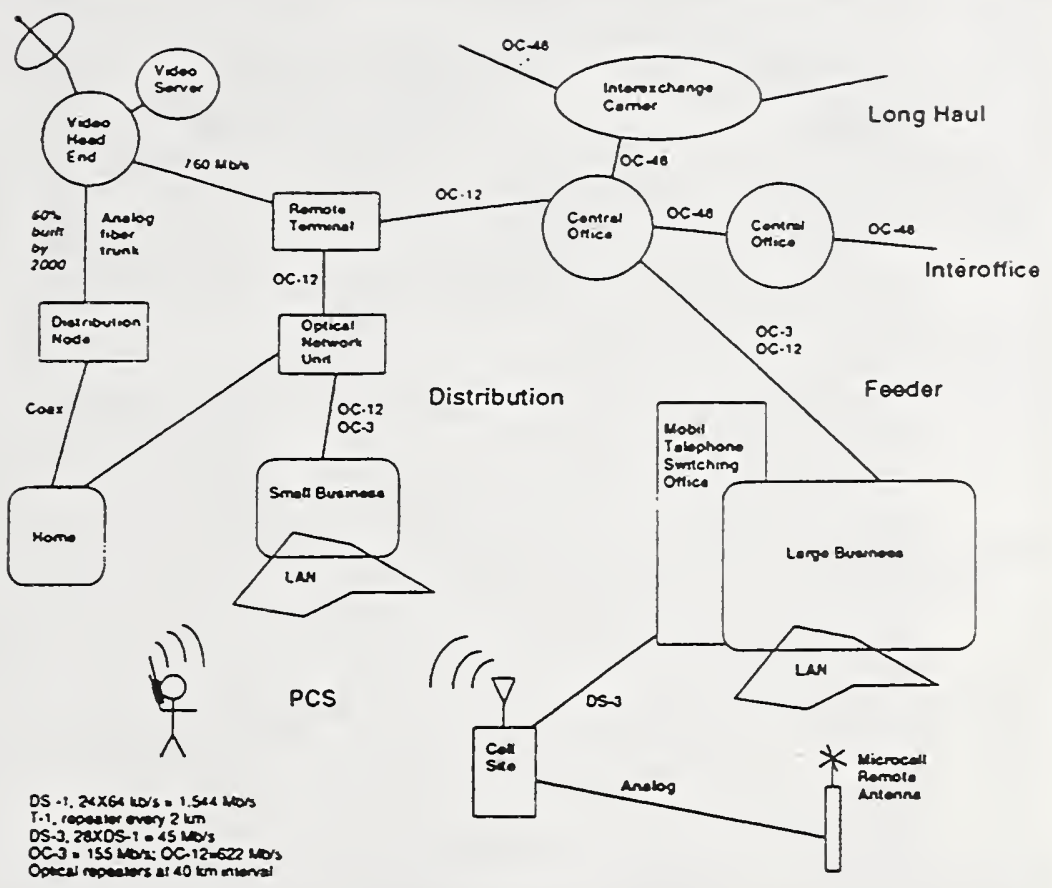

Nor

\section{Evolving Areas of OE Utilizacion}

$\diamond$ LAN development - $>50 \%$ will be wireless

$\diamond$ Home information network - possibiy separate from LAN - - Information appliances

- Audio, video, banking, home control, PCS

- High speed facsimile

$\diamond$ WAN development

- Extension of optical LAN (latency an issue)

- ATM cell traffic carried by SONET transport 


\section{Evolving Areas of OE Utilization (Cont.)}

- Telecommunications

- Video dial tone service

- Optical fiber access to network moving nearer the home and into businesses

- Homes may have customer owned optical fiber terminals

- Mobile Telecommunications

- Optical fiber feeders to MTSOs and cell sites

- Optical fiber provides analog links to remote antenna sites

\section{Evolving Areas of OE Utilization (Cont.)}

- Communications distribution

- Remote optical terminals and pedestals

- Hostile environment

- Power is an issue

- Vehicular applications

- Optical data buses in ships, planes and cars

- Optical sensors (gyros, pressure, fluid level, temperature, distance, etc.)

- Laser range finder, IR vehicle roadway positioners, smart highway systems 


\section{Evolving Areas of OE Utilization (Cont.)}

- Memory / data-storage systems

$-2 \mathrm{hr}$ movie needs about $43 \mathrm{~Gb}$ ( $<10 \mathrm{~s}$ at OC-12)

- Read / write / erase - array enhanced

$\checkmark$ Processor interconnects

- Multiprocessor cluster connection to arrays of direct access storage devices

- Eliminate the bottleneck of slow, interference prone electronic interconnects

- Software bottleneck with conventional processor architectures calls for new approaches with high bandwidth interconnects

\section{ATM Switch Interconnections}

- Optical backplane required

- $16 \times 161.2 \mathrm{~Gb} / \mathrm{s}$ ATM requires back plane with over $200 \mathrm{~Gb} / \mathrm{s}$ capacity

- If assume:

$-1 \mathrm{~Gb} / \mathrm{s}$ on each fiber

- 5 fiber module

- 100 fibers $=4100$ wires at $50 \mathrm{Mb} / \mathrm{s}$
External Interface Modules

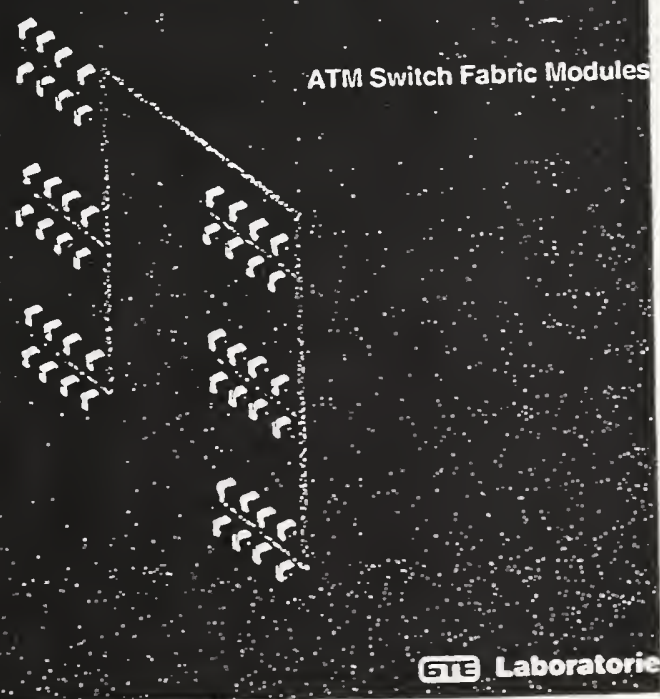




\section{Evolving Areas of OE Utilization (Cont.)}

- Transparent optical networks - a distant dream?

- PICs, WDM components

- Optical switching

- OE Sensor Improvement

- IR Imaging (plant tissue and textiles)

- Absorption (medical, environmental, air quality, process control)

- Compact laser scanners - laser arrays

- Optical fiber gyros

\section{Evolving Areas of OE Utilization (Cont.)}

- Printers (electrophotographic)

- low-cost short wavelength laser diodes and LED optical sources (arrays)

- No current US supplier

- Displays

- Flexible and portable for digital appliances applications

- Integrate drive electronics and display 


\section{Evolving Areas of OE Utilization (Cont.)}

$\Leftrightarrow$ Metrology and Inspection

- Improved optical sources

- Videometrics

- Higher resolution CCD arrays

\section{Silicon Waferboard}

Silicon Waferboard is an approach that utilizes silicon as a platform for hybrio integration of optoelectronic, electronic, and optical components

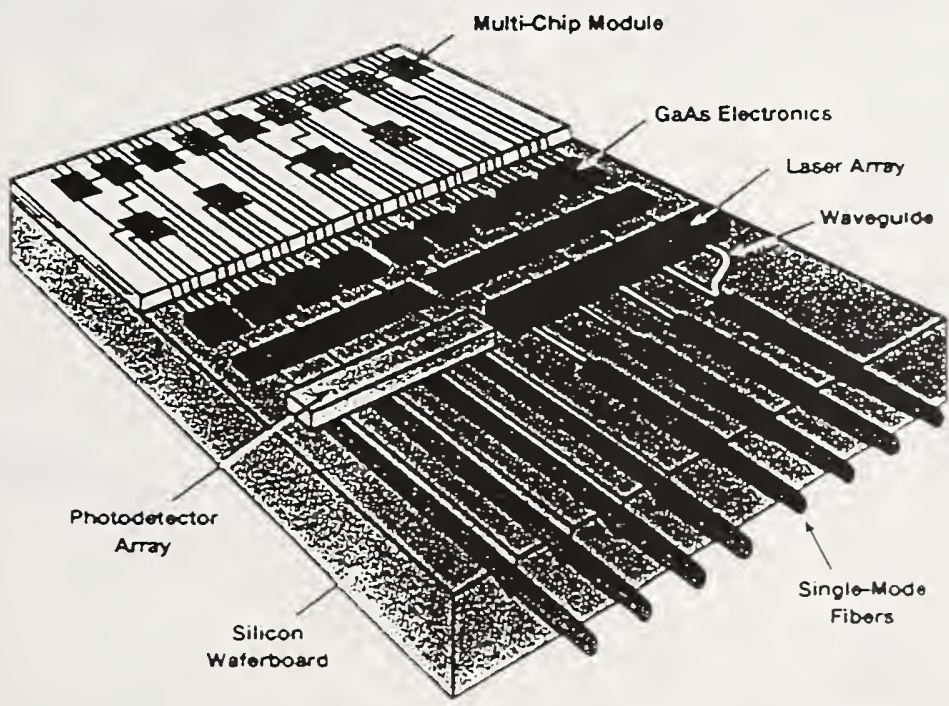




\section{Silicon Waferboard Example}

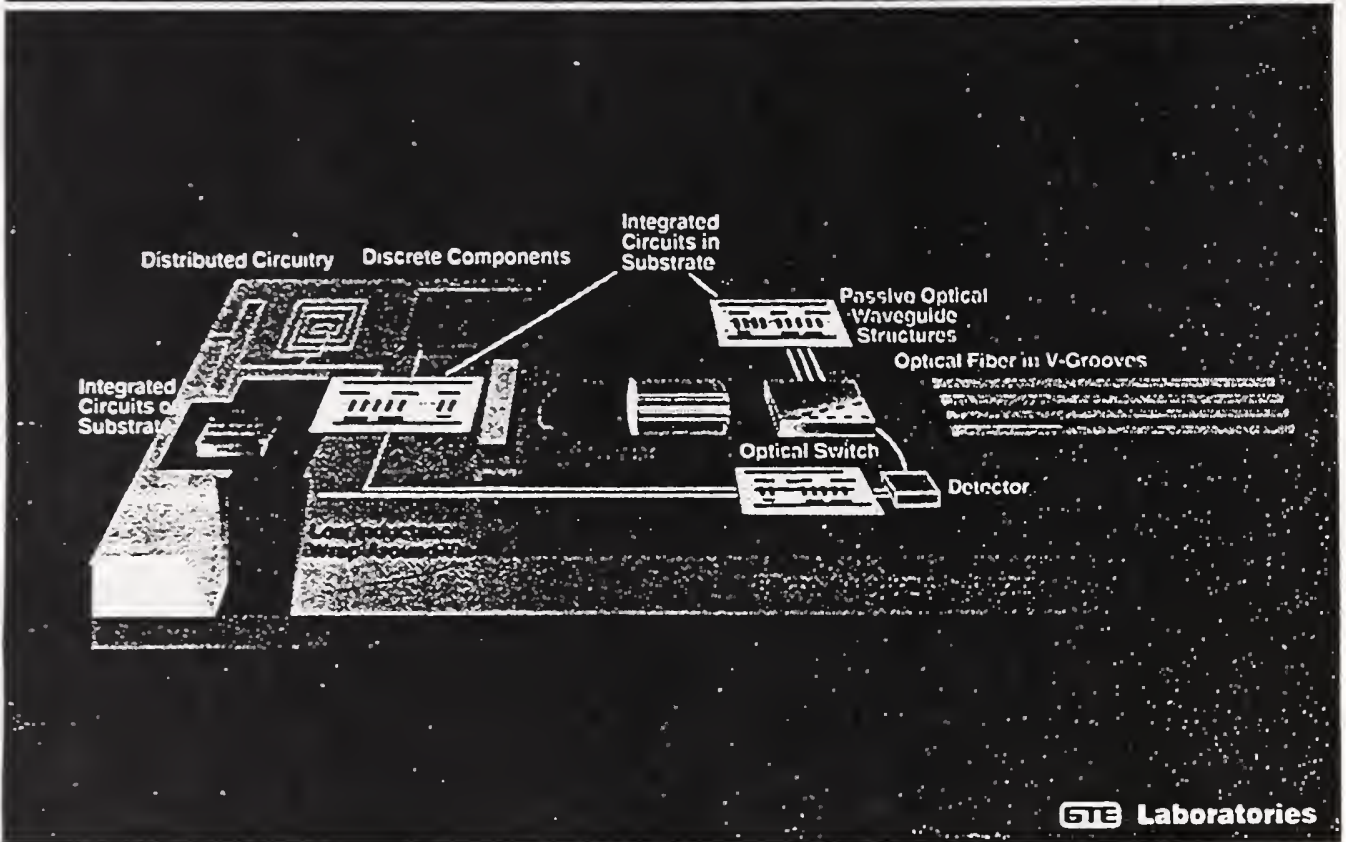

\section{Laser Passive Alignment on Silicon Waferboard}

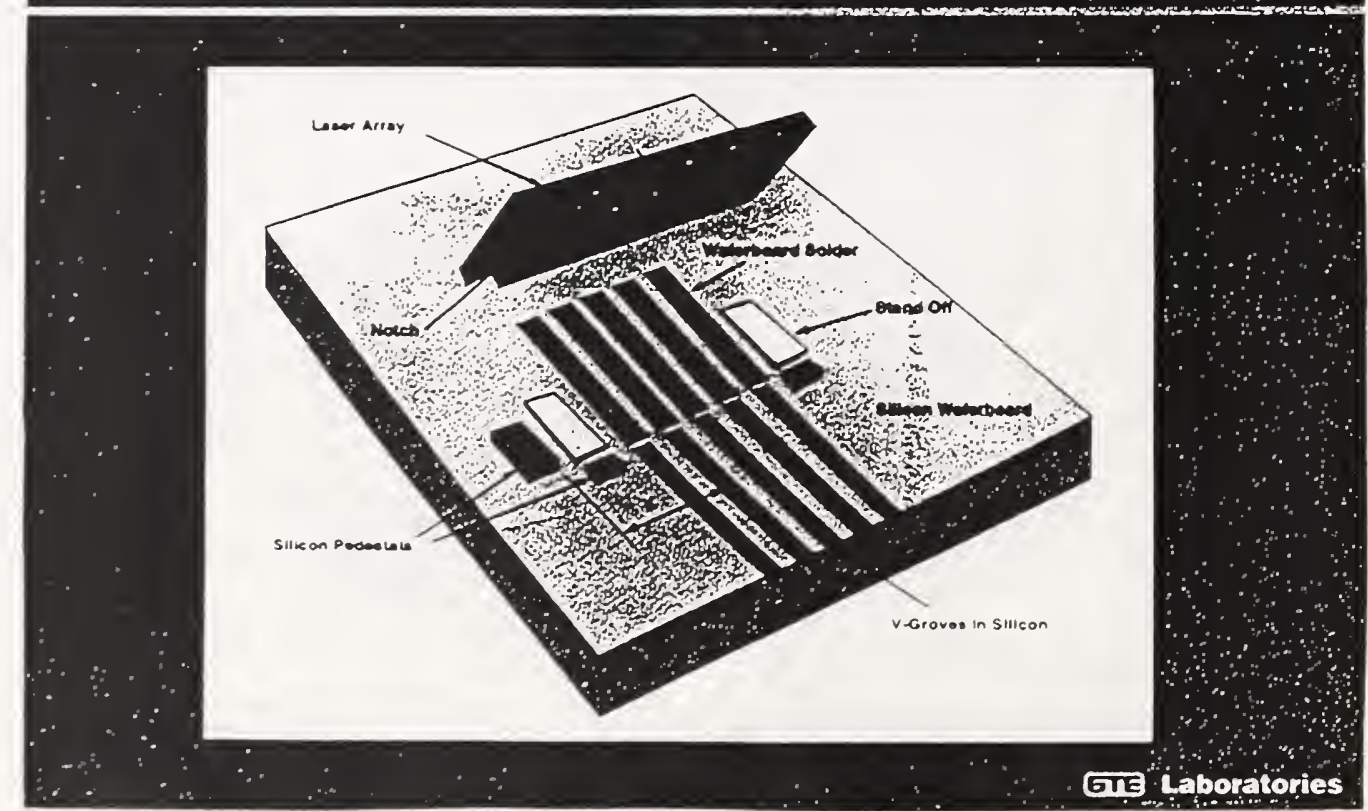




\section{4-Channel Silicon Waferboard Transmitter}

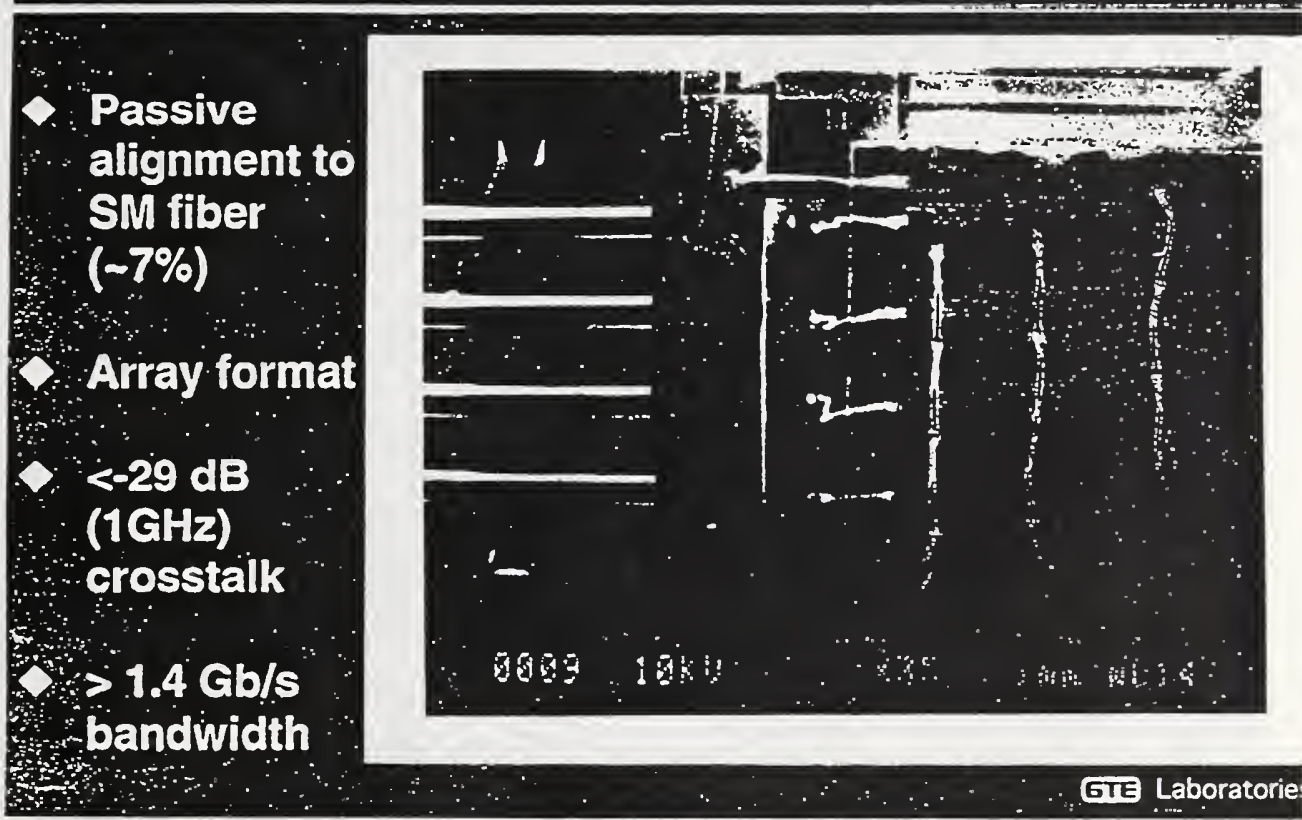

\section{Challenges for Consideration}

- Robotic control

- Placement accuracies $<1 \mu \mathrm{m}$

- Sensitive tactile sensors

- High resolution machine vision

- Improved accuracy and precision of submicron measurements over large distances

- Polymer coatings for reliable environmental protection

- New glass hosts for $1.3 \mu \mathrm{m}$ optical waveguide amplifiers 


\section{Challenges for Consideration (cont.)}

- Materials for green and blue LEDs and lasers for optical storage, printers, and displays

- Better polymers for passive and active optical components

- Optical couplers

- Mode transformers

- Optical switches

- Optical modulators

- Optical memory media

\section{Challenges for Consideration (cont.)}

- Large-area, thin-film process control for uniformity

- Extrusion processes and materials for polymer film deposition over large areas ( 36 in $\times 36$ in)

- Lateral deposition and epitaxial growth control techniques

$\checkmark$ Integrable optical isolators

- Materials and processes for VCSELs and other efficient, long-wavelength lasers and improved MSM detectors 


\section{Challenges for Consideration (cont.)}

- Improved resolution CCDs and IR sensors

- Advancements in chemically sensitive OE sensors

- Improved materials and process control techniques for polarization insensitive waveguide switches

- Improved techniques for fabricating good optical switches with moderate (50 - 100 ns) switching speeds

\section{Grand Challenge:}

Reduce the costs involved in fabricating and particularly packaging optoelectronic components to allow continued improvement in our quality of life and the health of our economy.

\section{A Difficult Job -}

- Broadly multidisciplinary problems

- National infrastructure for packaging is shrinking

- Team work is the answer 


\section{Che-Yu Li, CoRnell University}



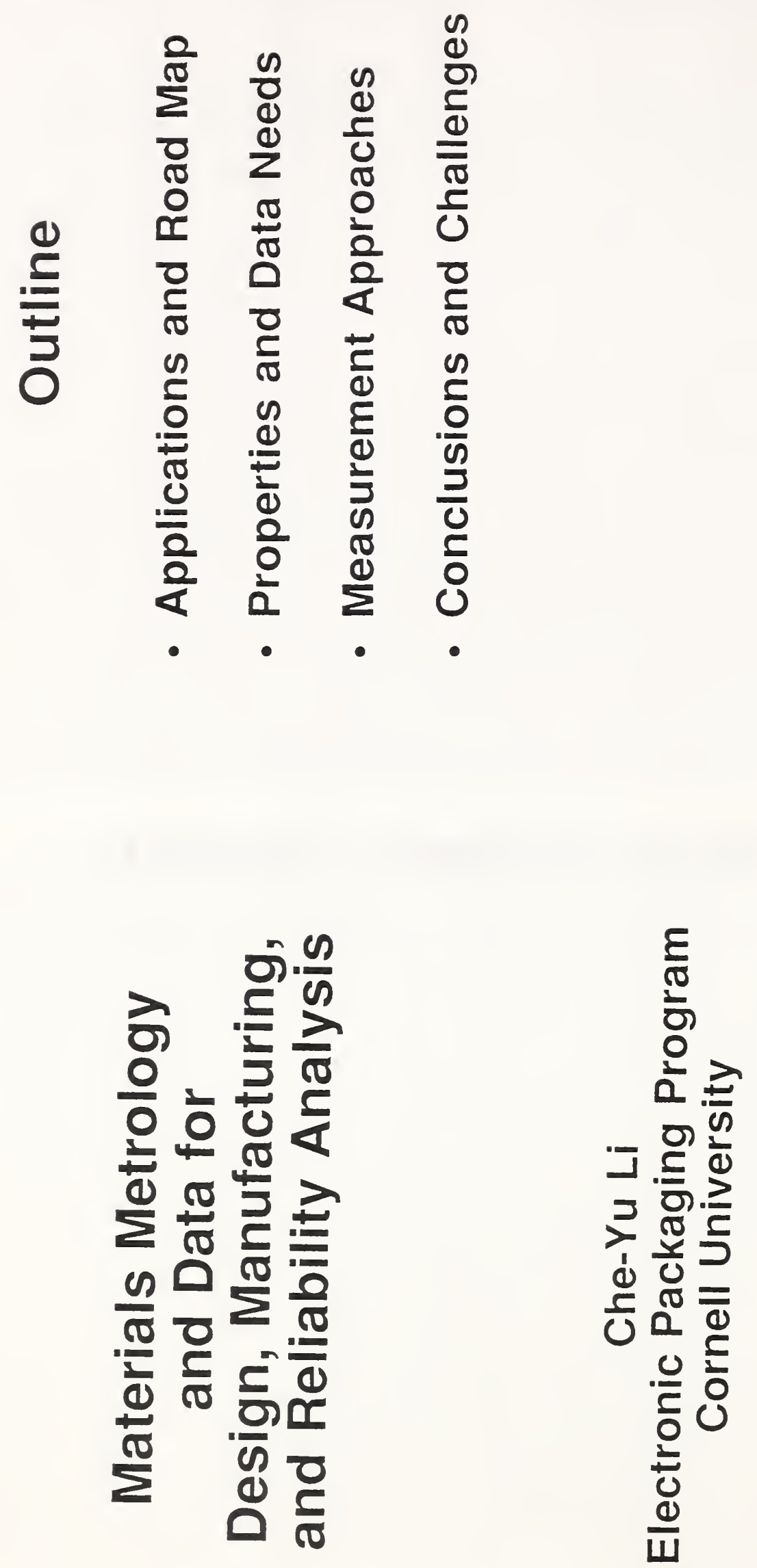


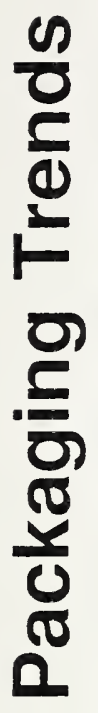

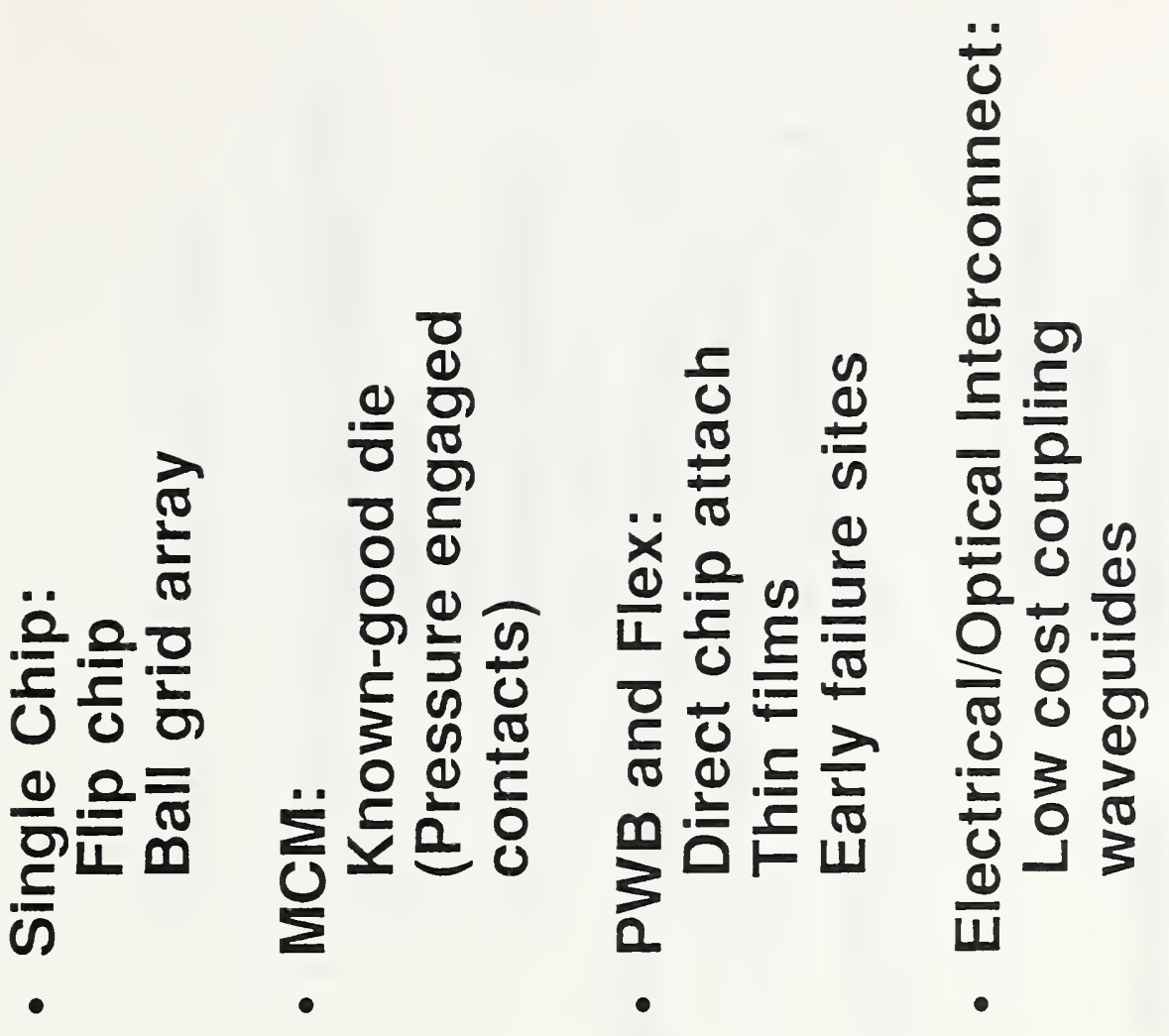

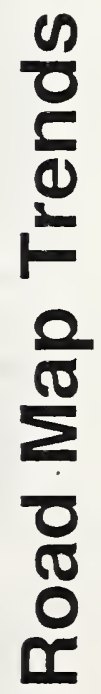

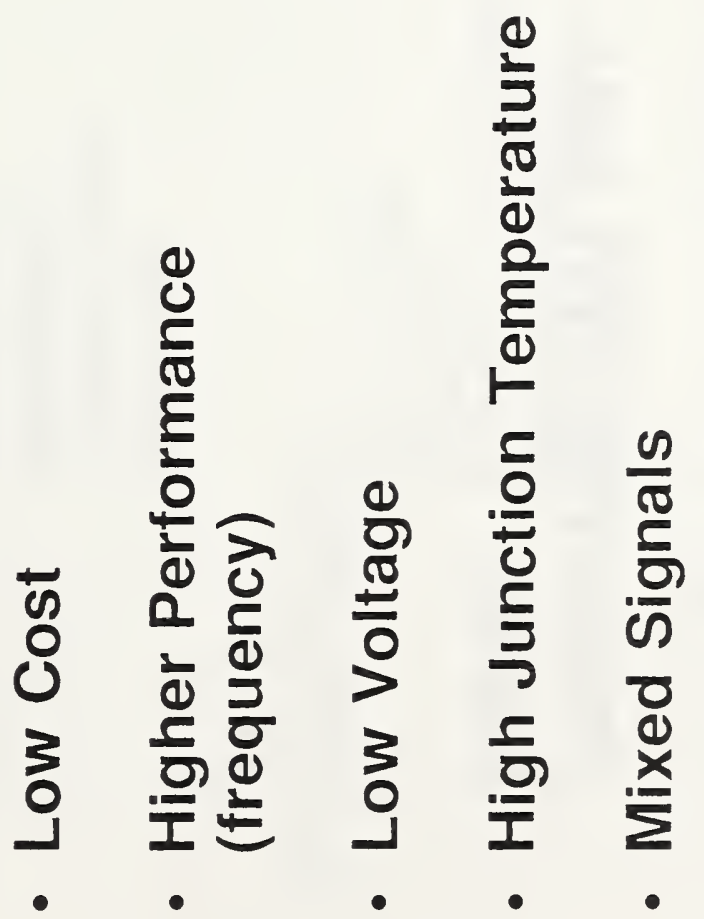



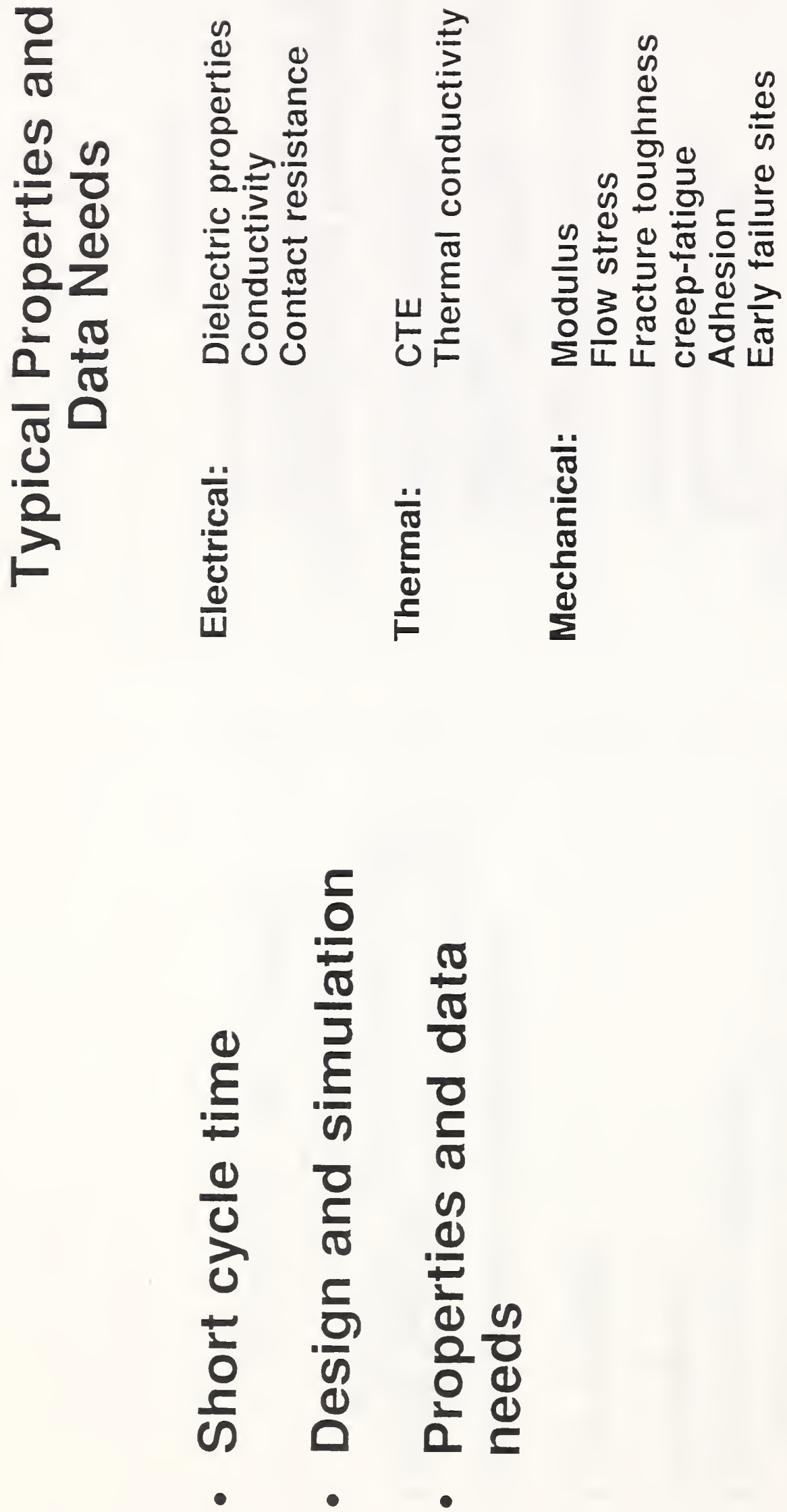

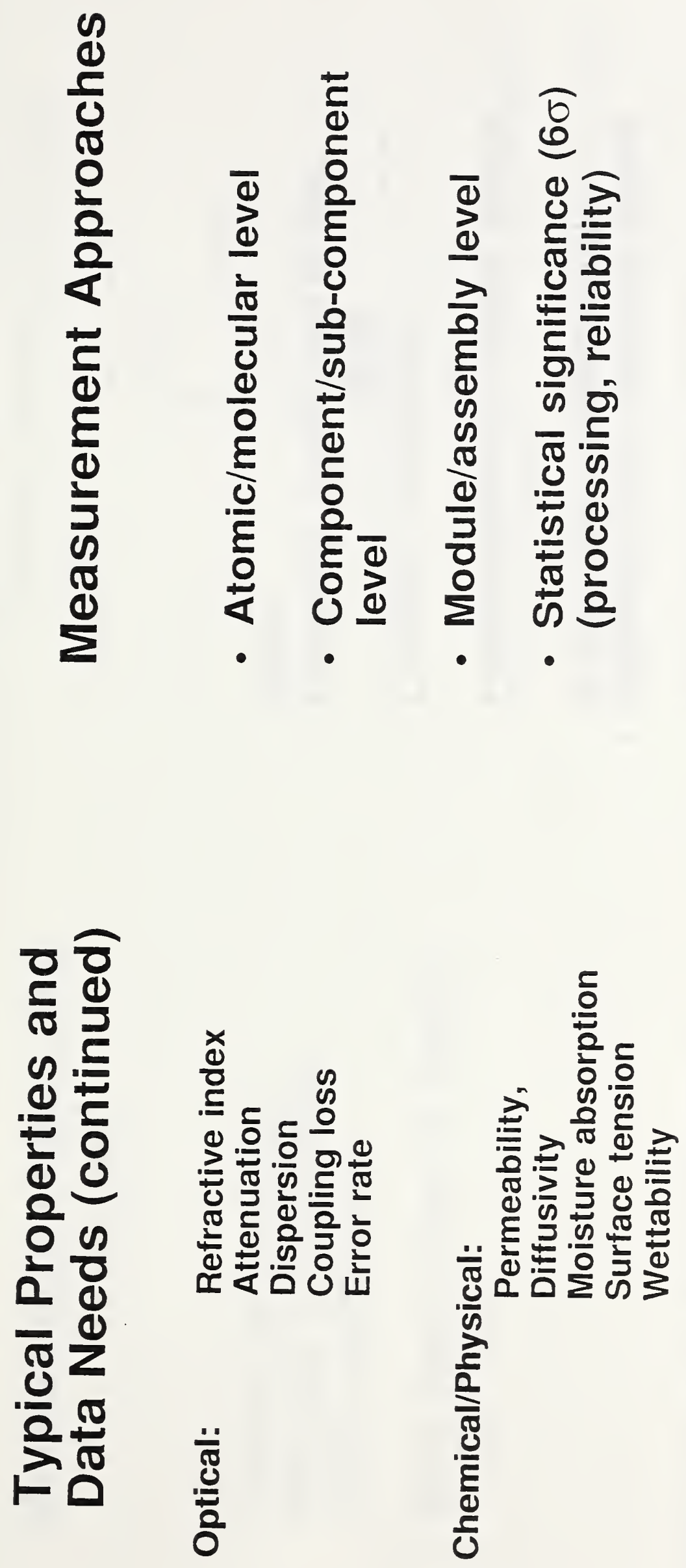

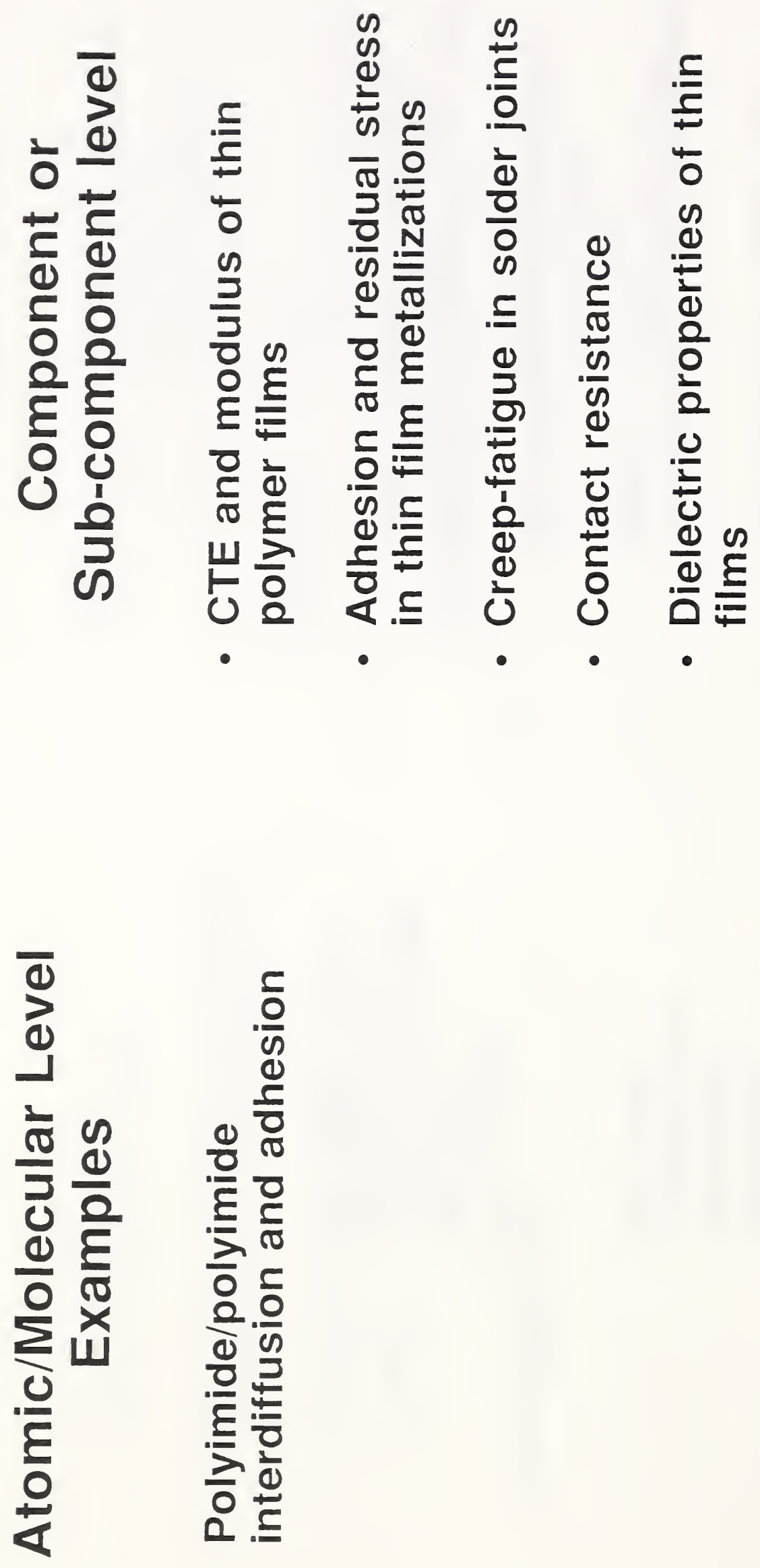

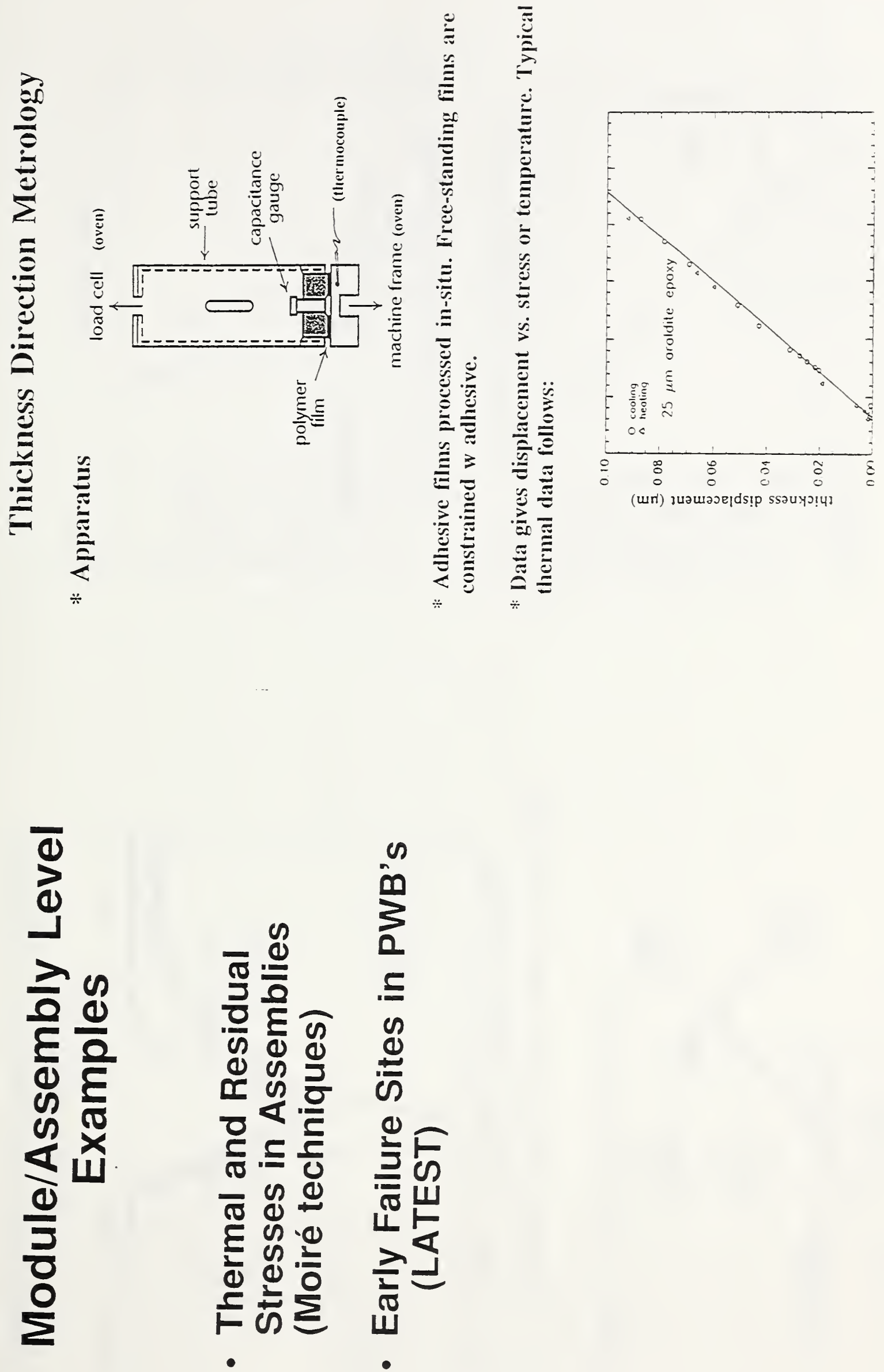

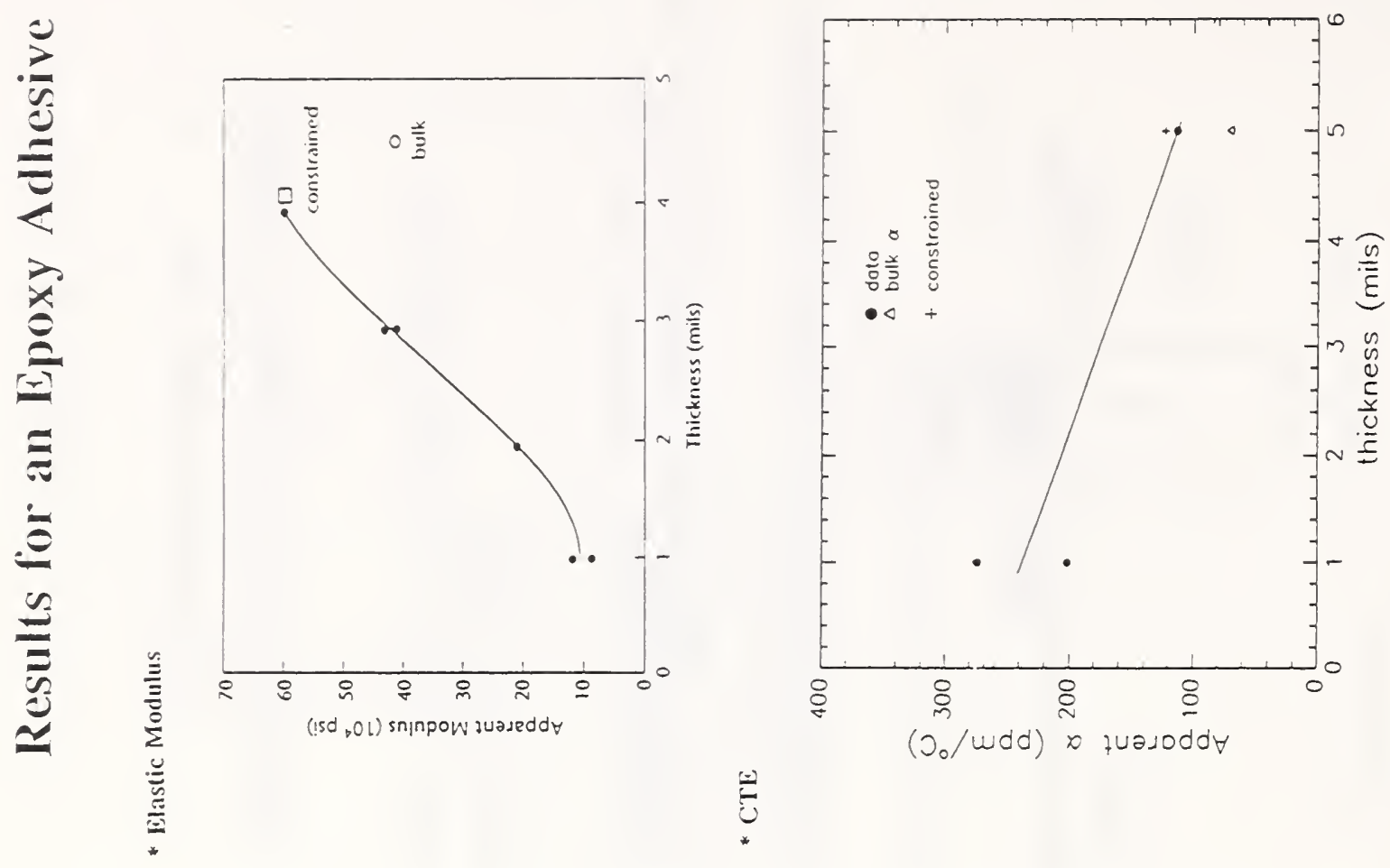

E

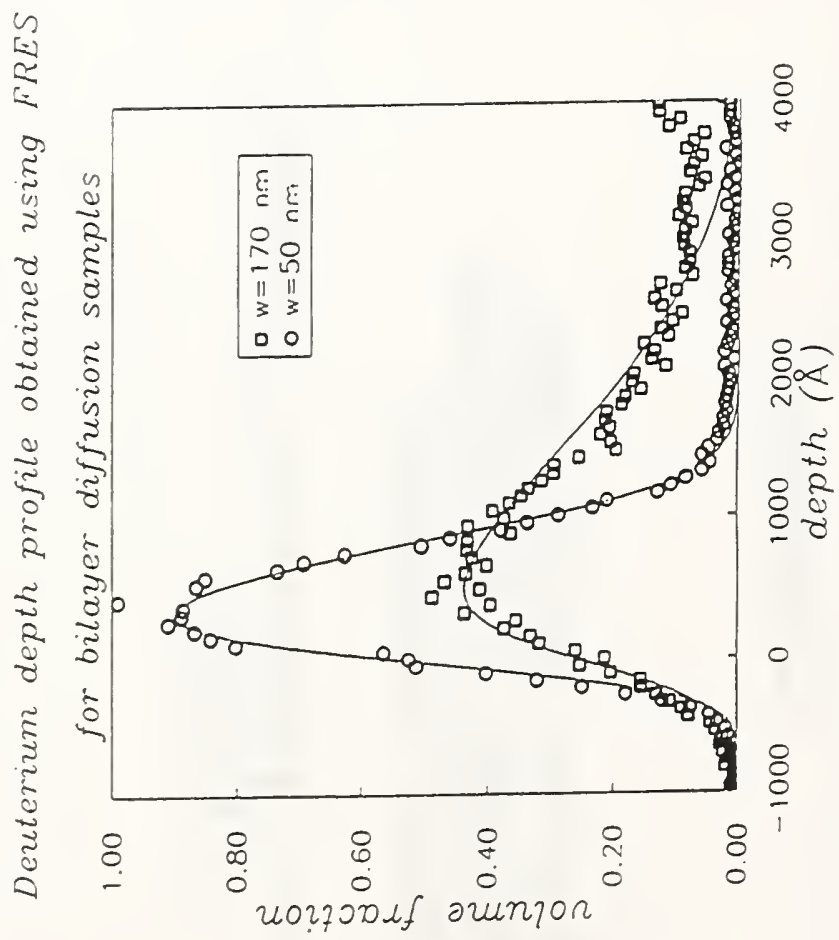



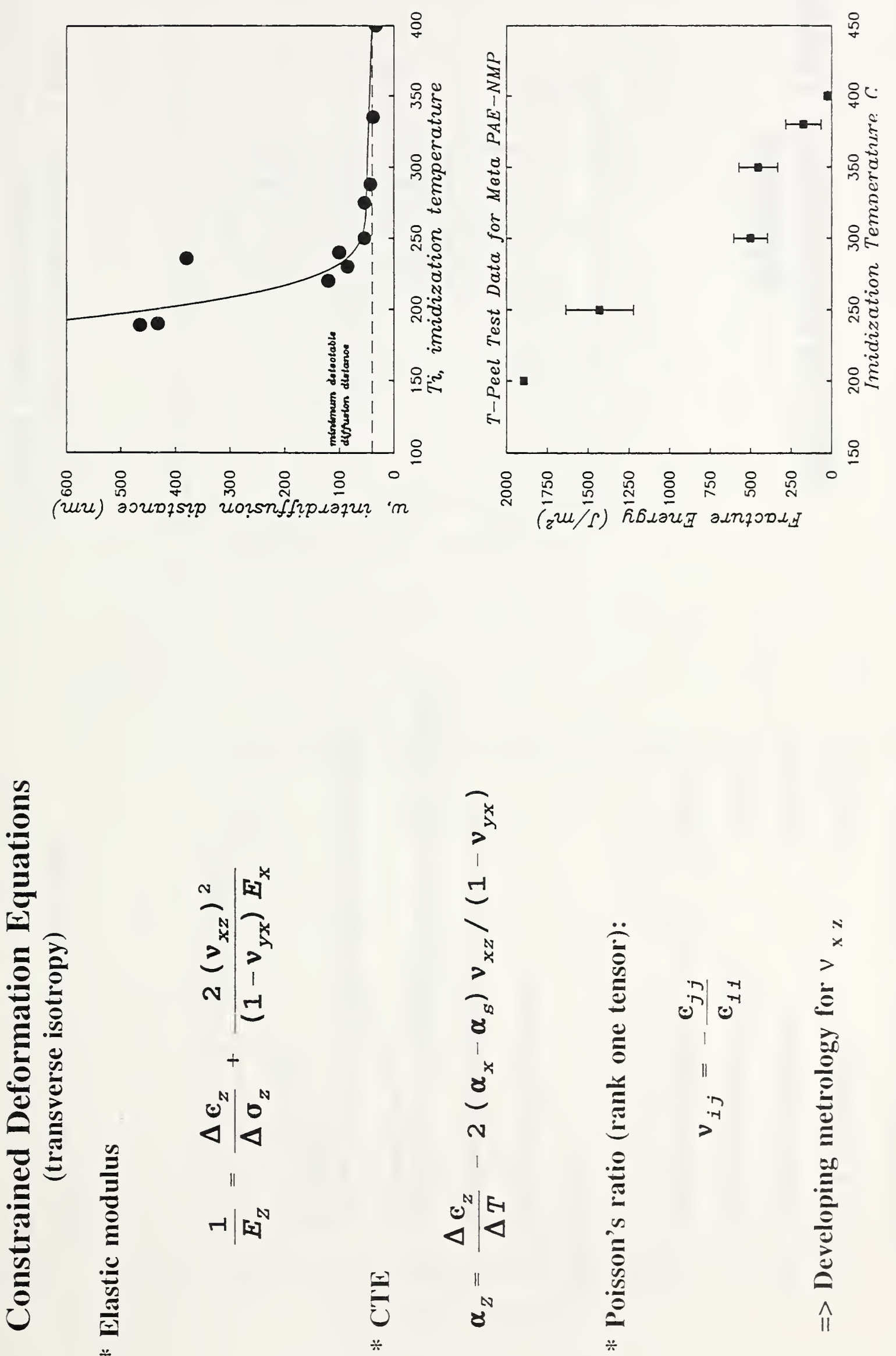

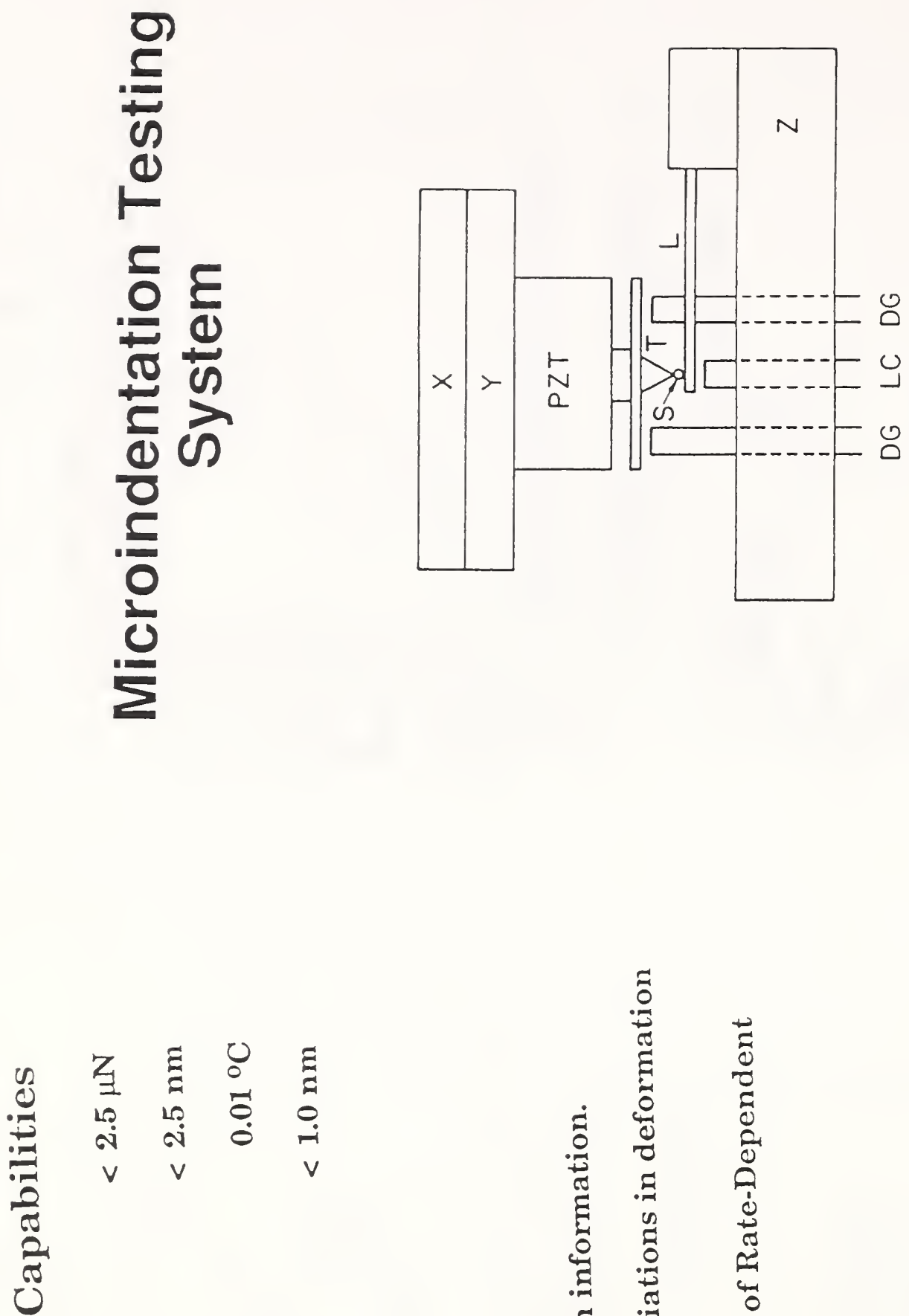

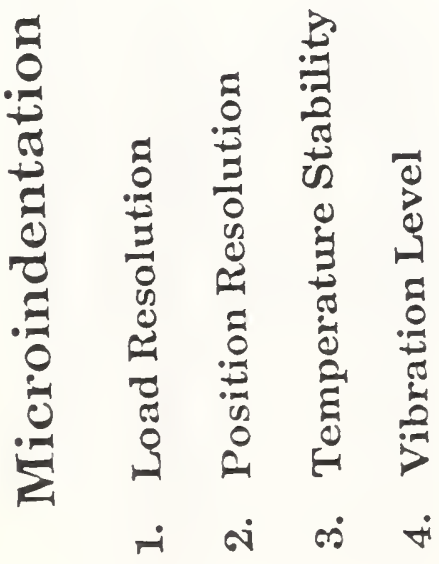

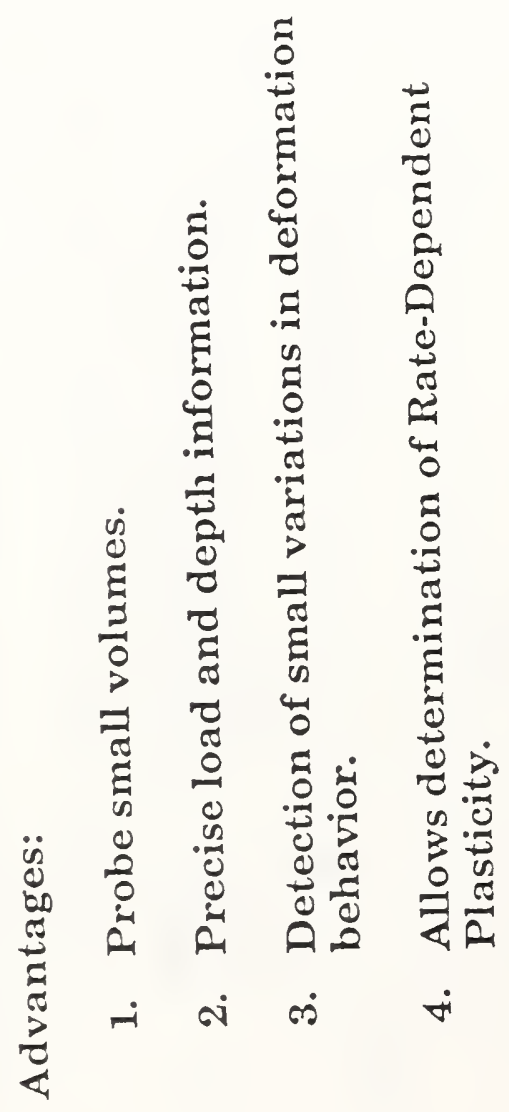



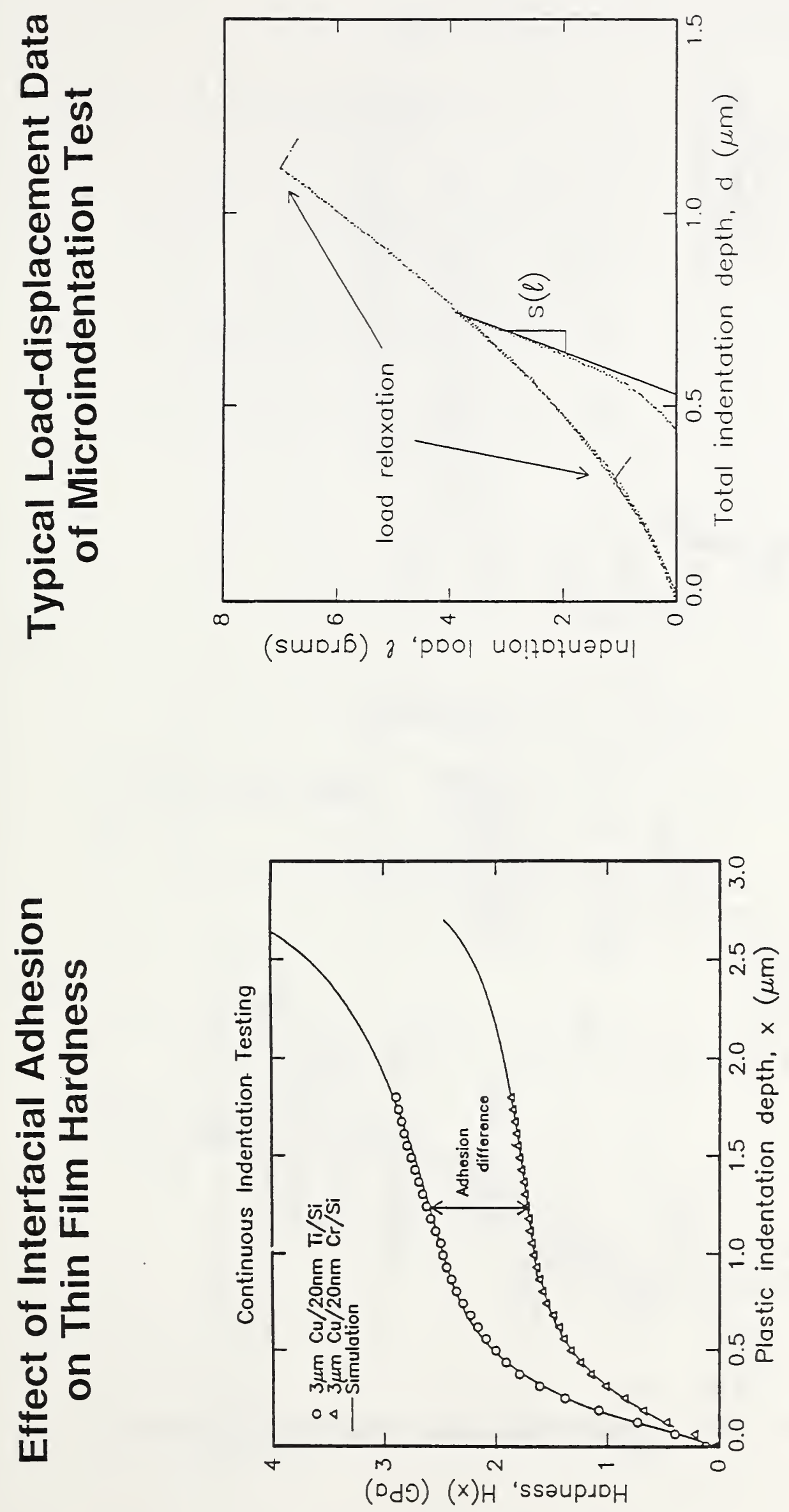

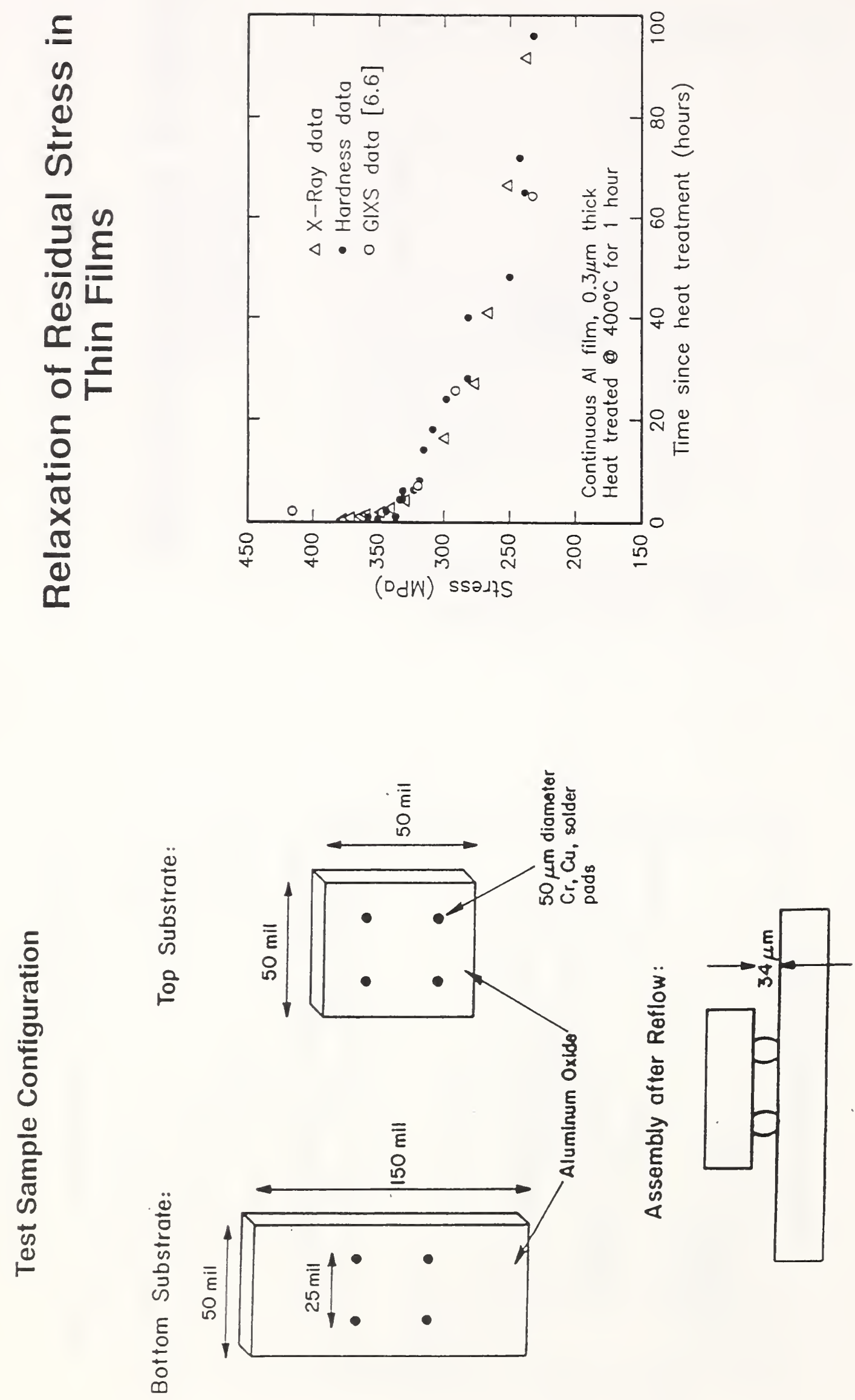


\begin{tabular}{|c|c|c|c|c|c|c|c|c|}
\hline 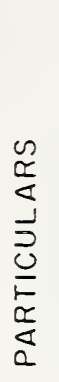 & $\frac{\text { o }}{\grave{2}}$ & 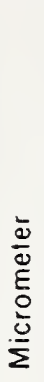 & $\begin{array}{l}\frac{0}{0} \\
\frac{0}{5} \\
\overline{0} \\
\frac{0}{0} \\
\stackrel{0}{0} \\
\frac{0}{1}\end{array}$ & 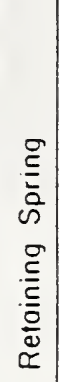 & 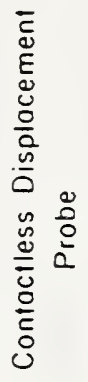 & 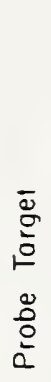 & $\begin{array}{l}\overline{\bar{\Delta}} \\
\bar{u} \\
\dot{\Delta}\end{array}$ & 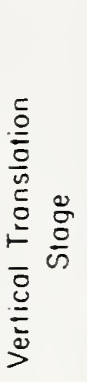 \\
\hline शं & - & $\sim$ & $m$ & $\sigma$ & $n$ & 0 & $\sim$ & $\infty$ \\
\hline
\end{tabular}
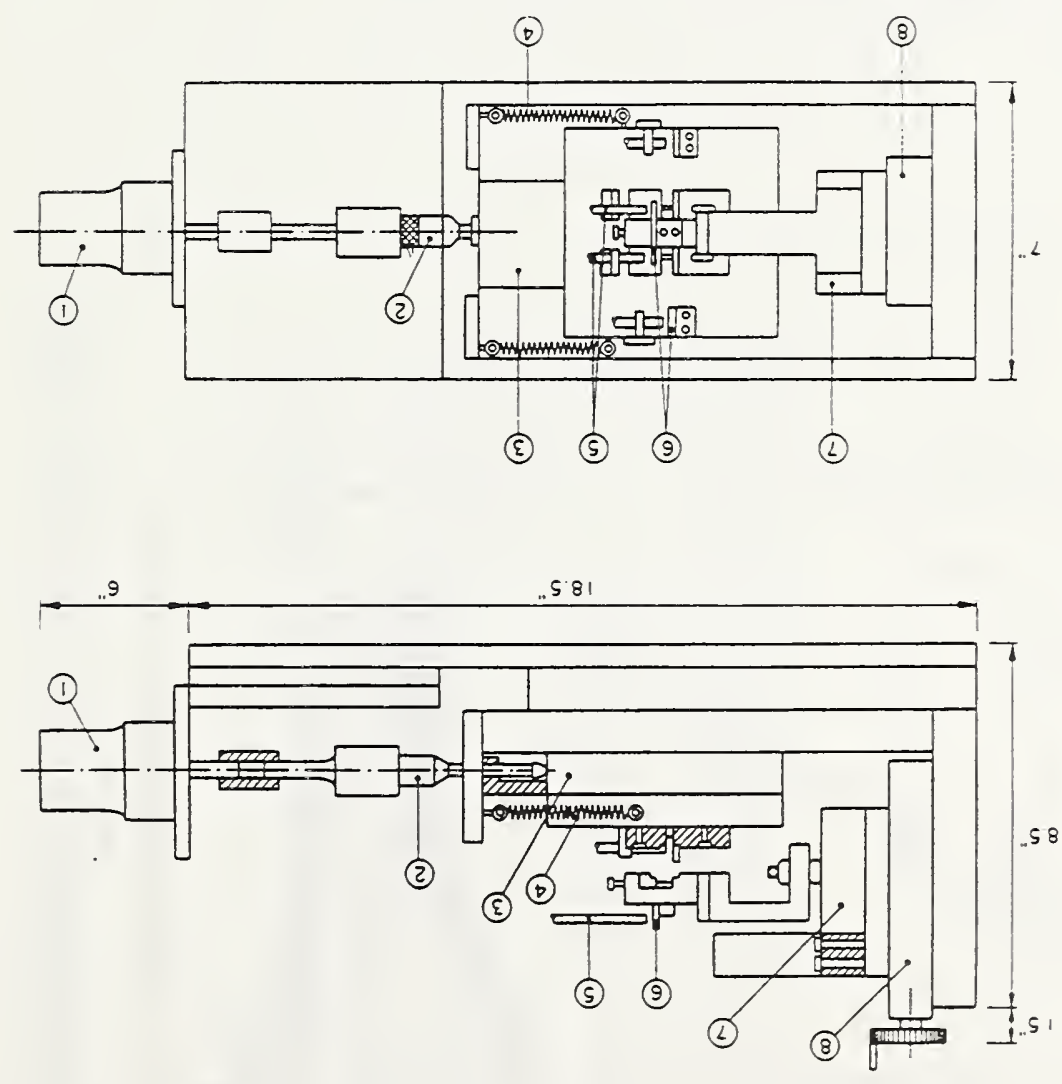

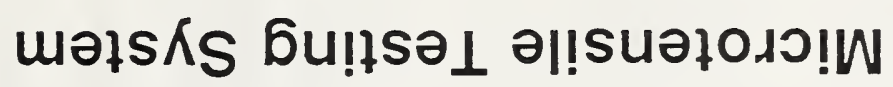



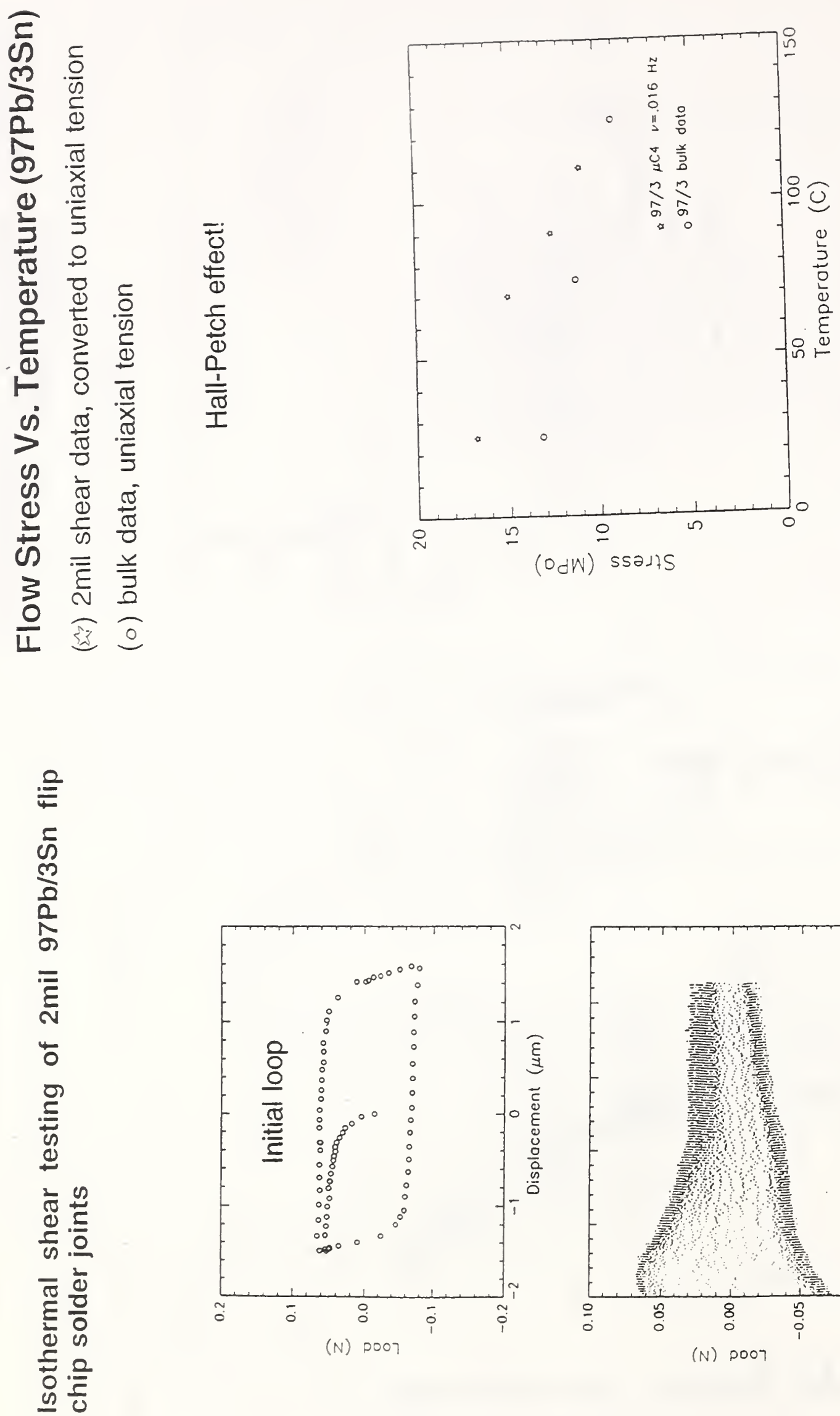

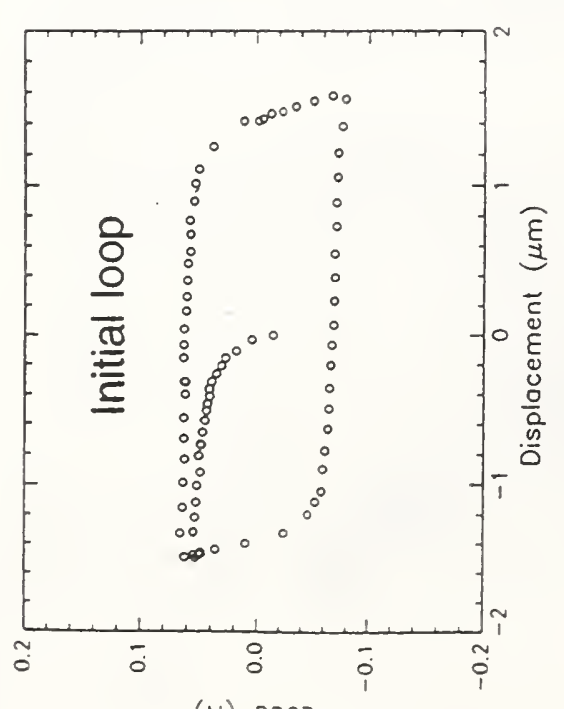

(N) 0007

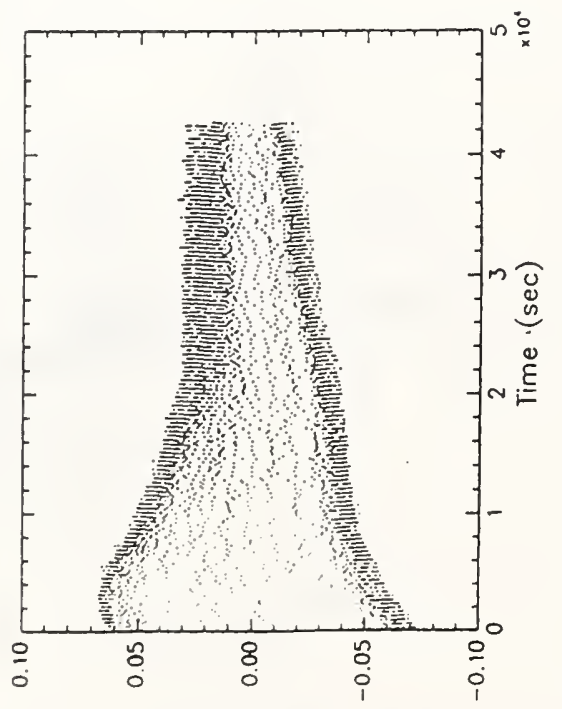

(N) 0007 

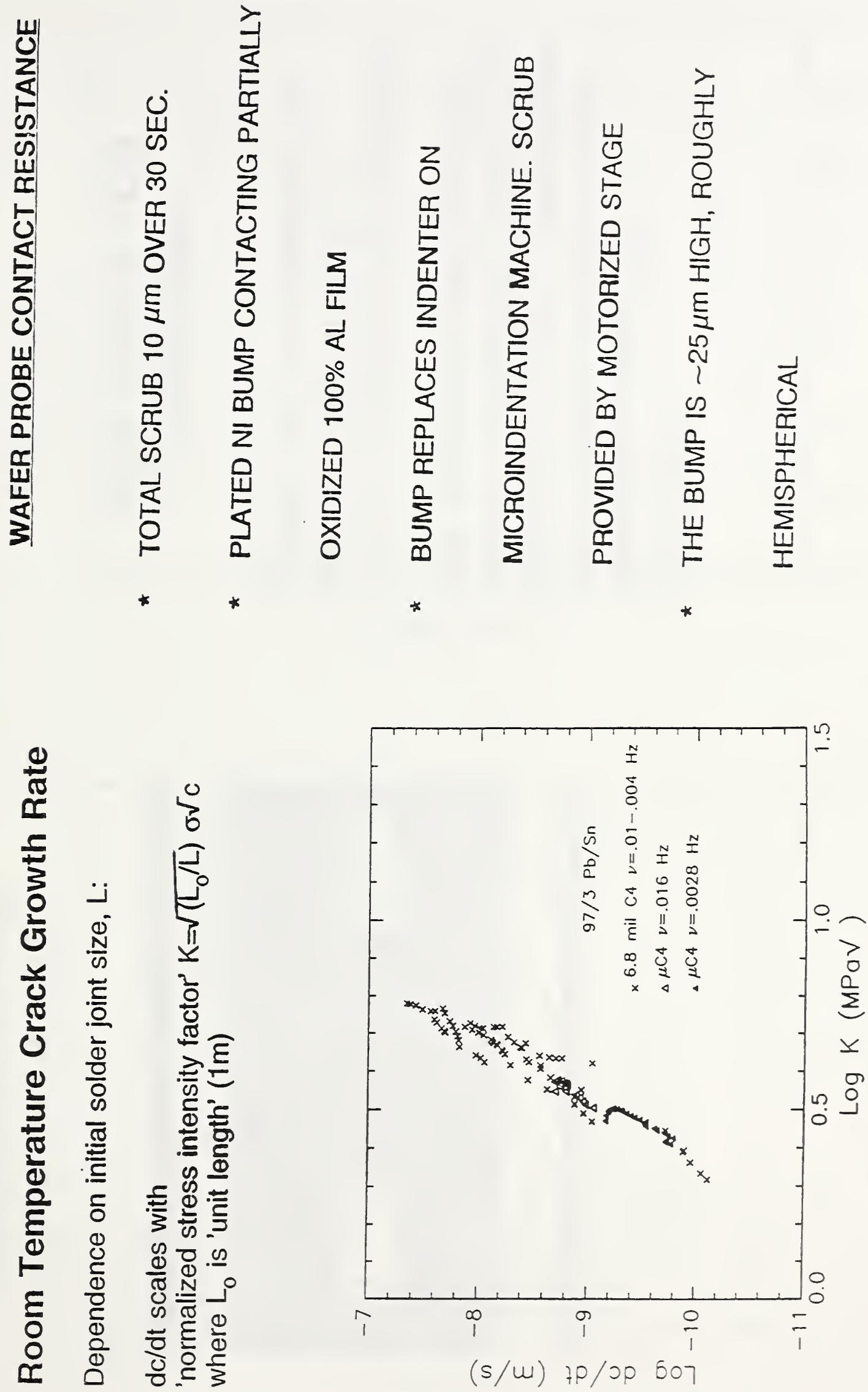

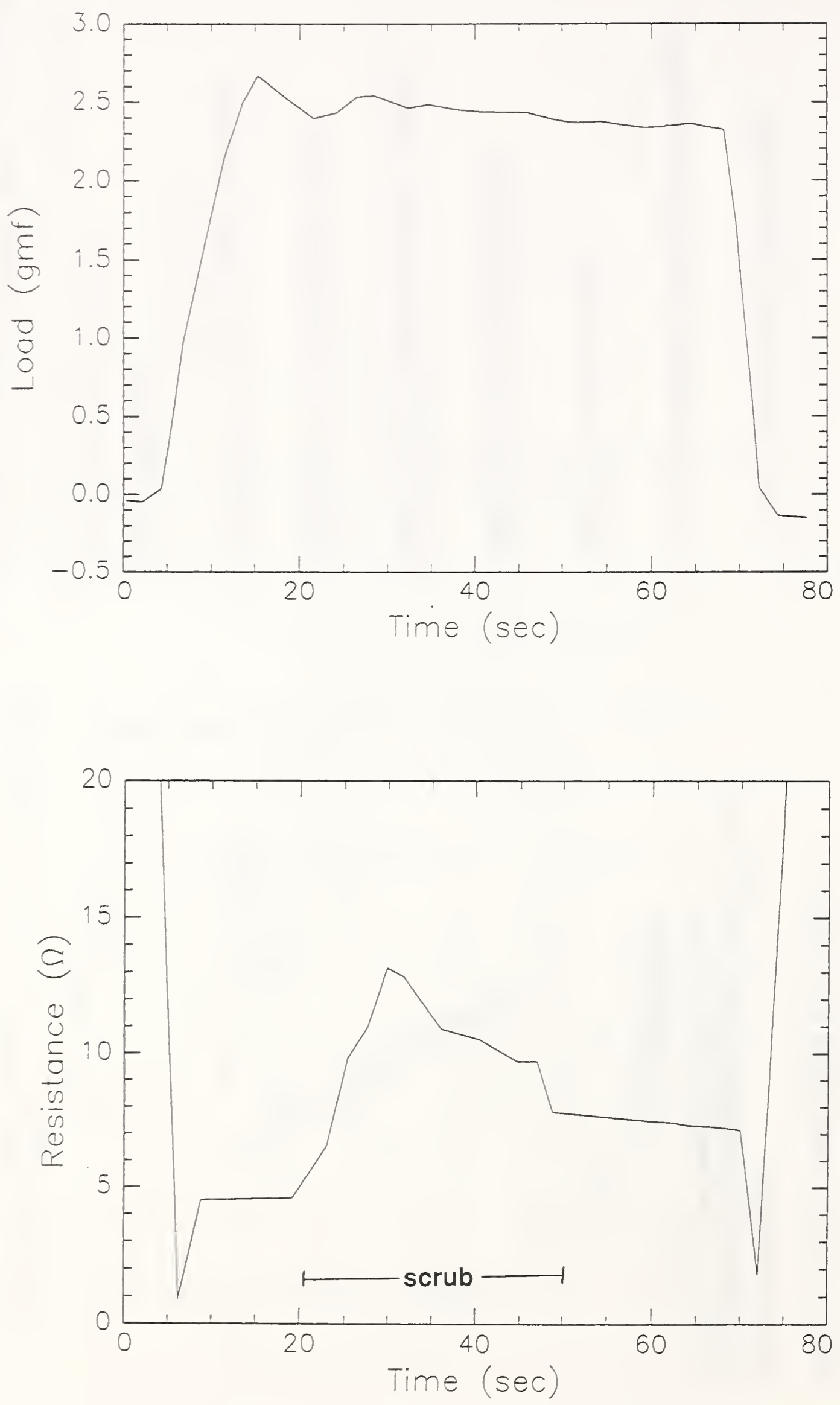

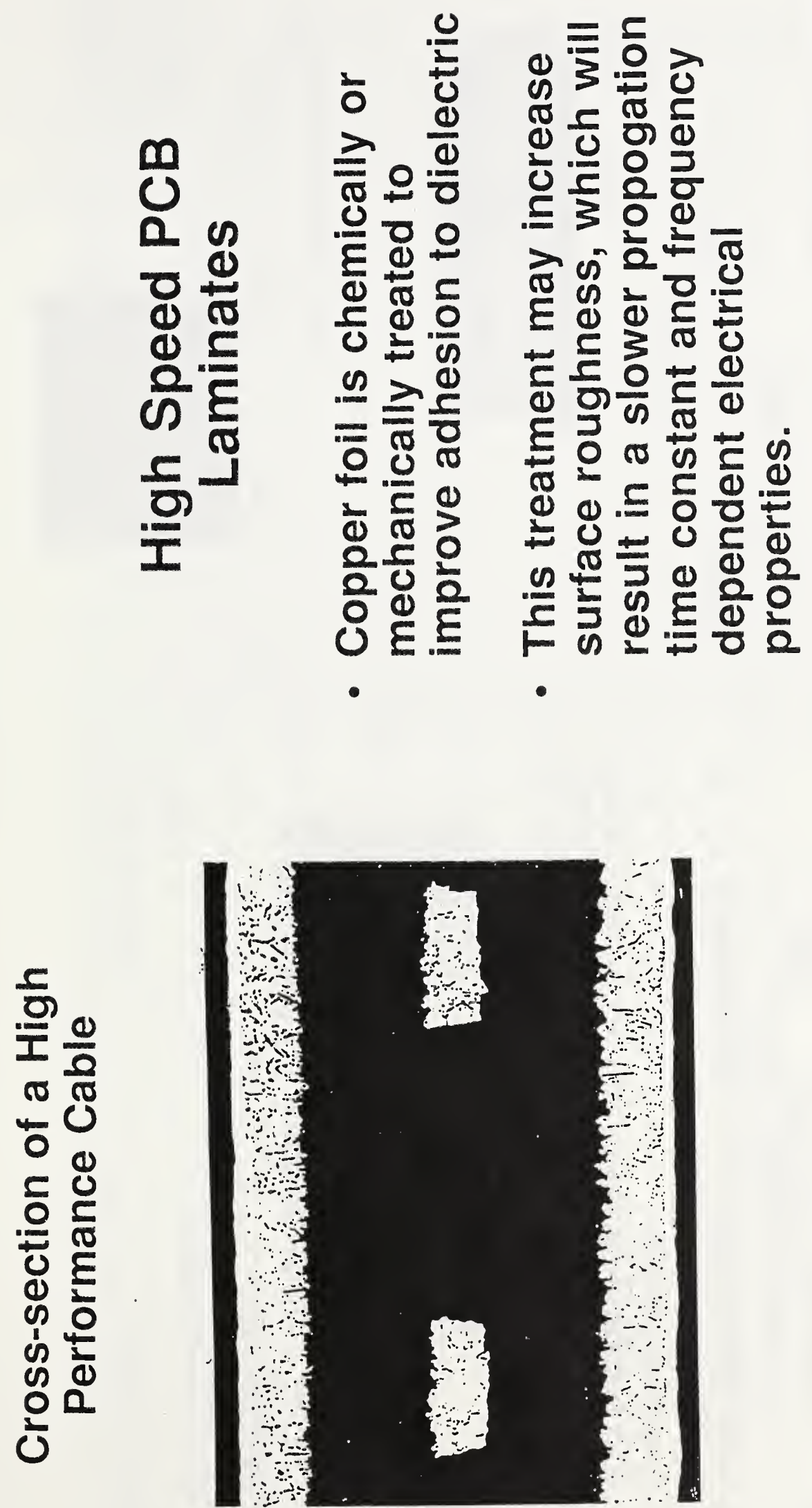

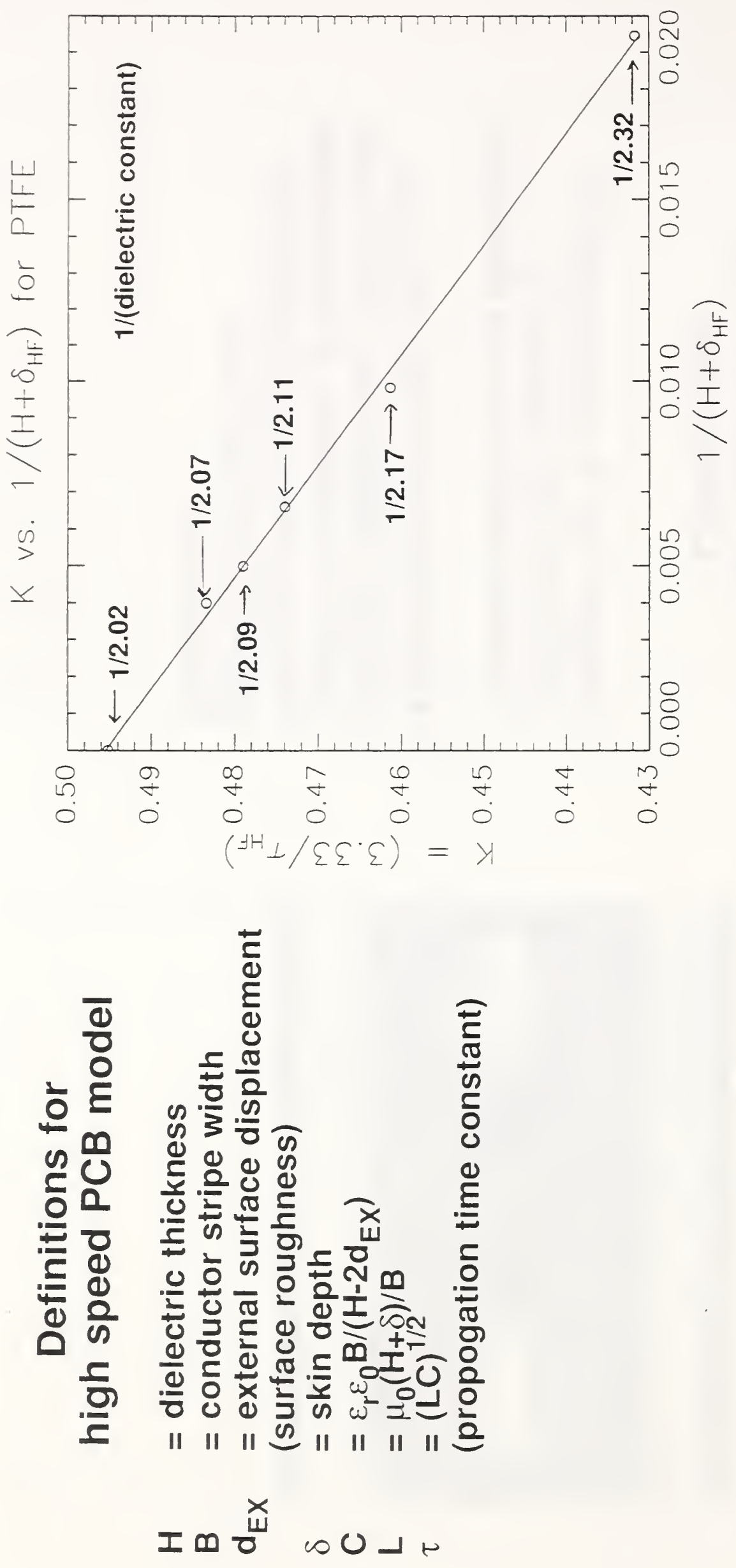


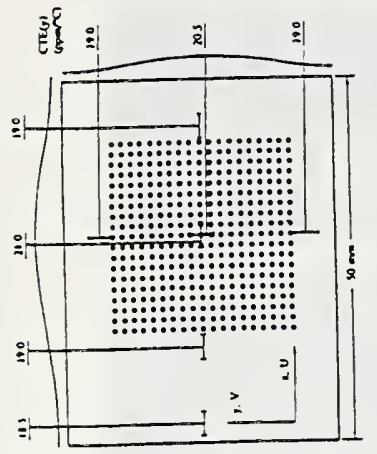

政
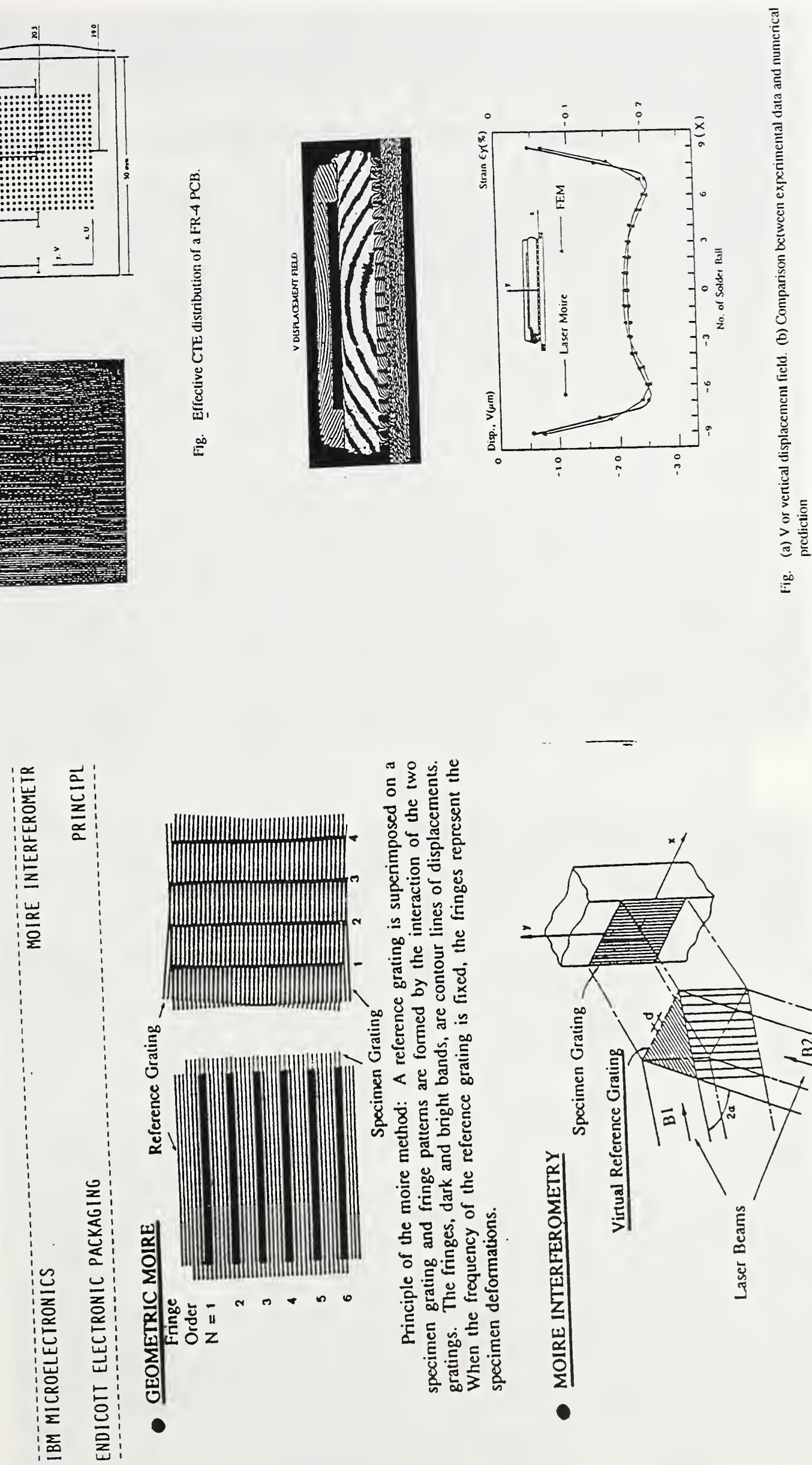

올

ఫั

을을

总总记

․ㅡㄹ

象号

跑分造远

顿岛.

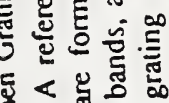

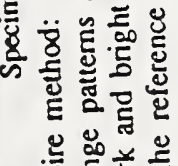

记导总

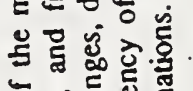

을

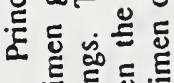

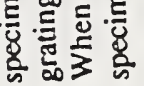

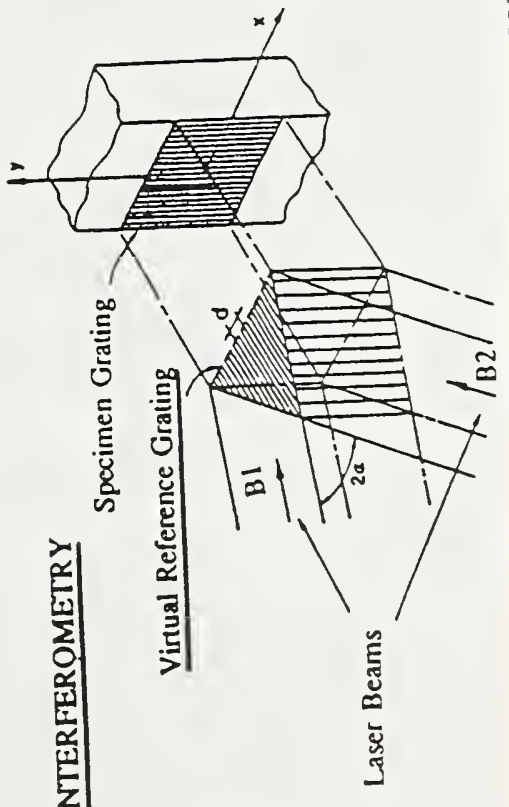




\section{Defect Signal versus Defect Constriction Resistance}

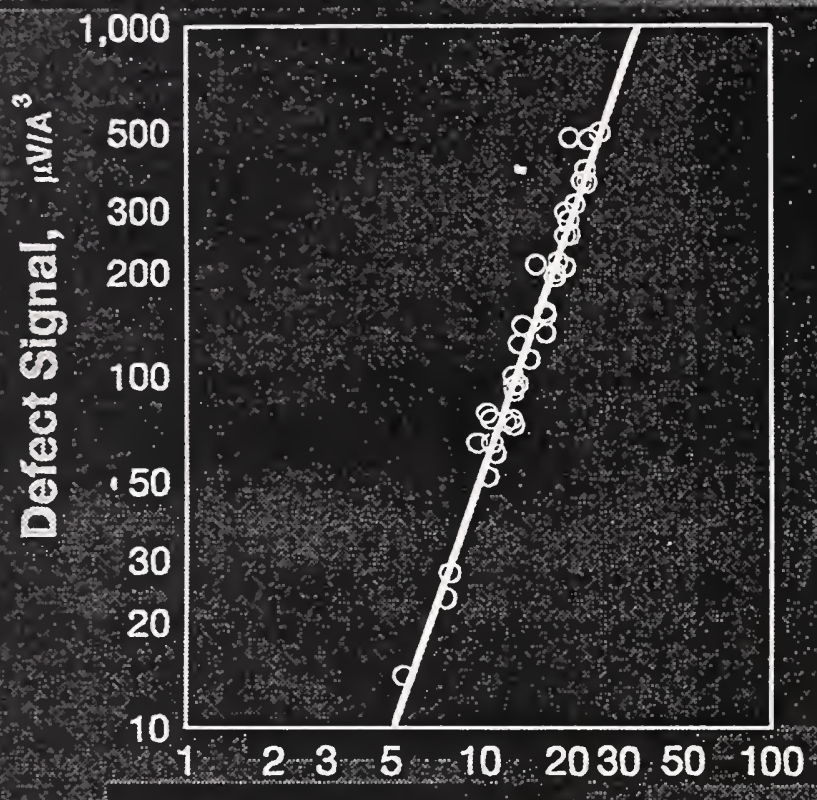
Constriction Res., mohm

\section{Phase Difference and Detection}
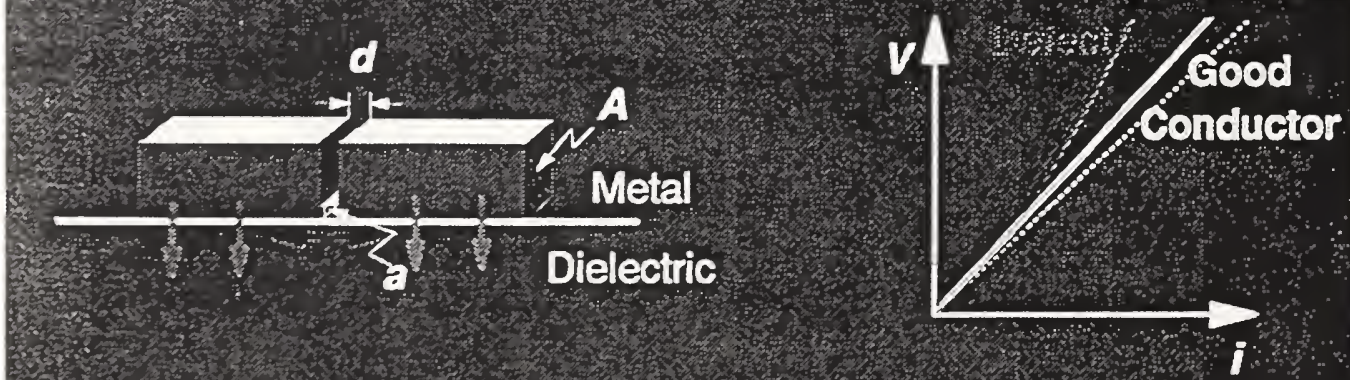

Defeot

Coos

Conductor

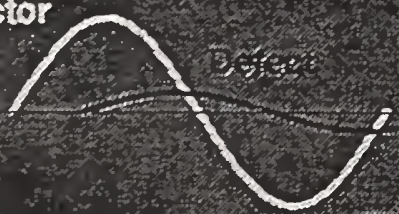

Output Signal

Motring

Signal from

Good Conductortsum 


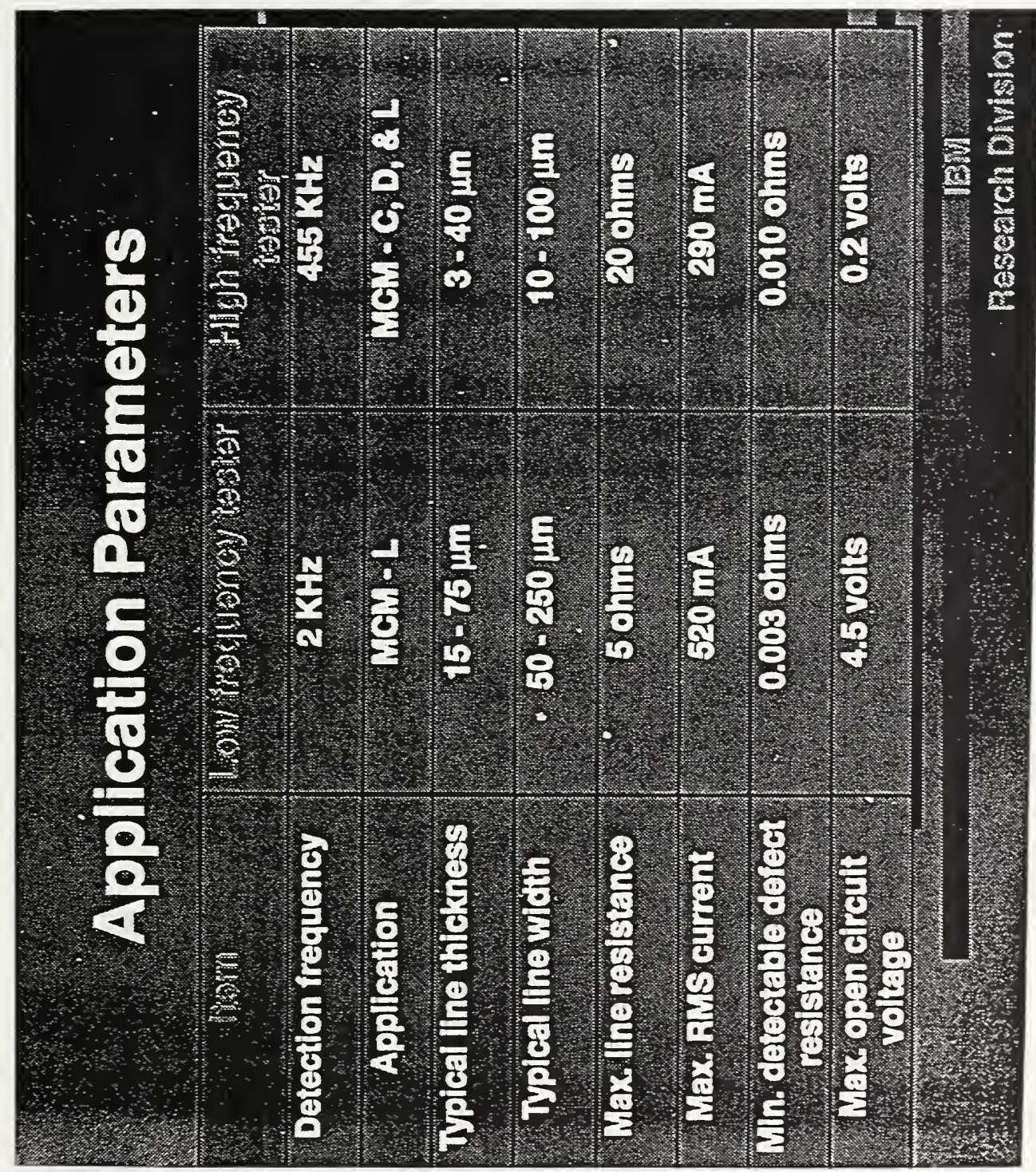



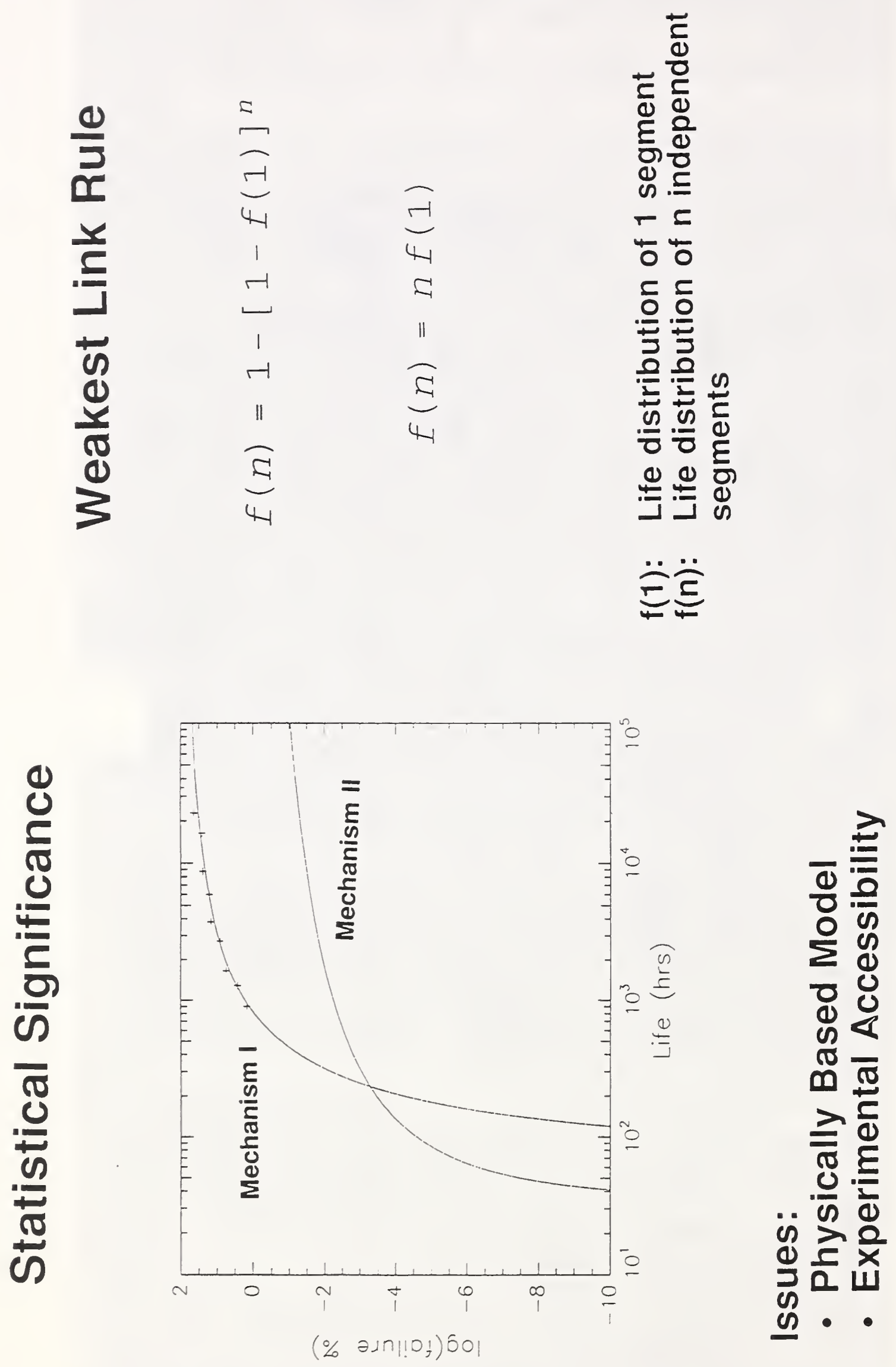

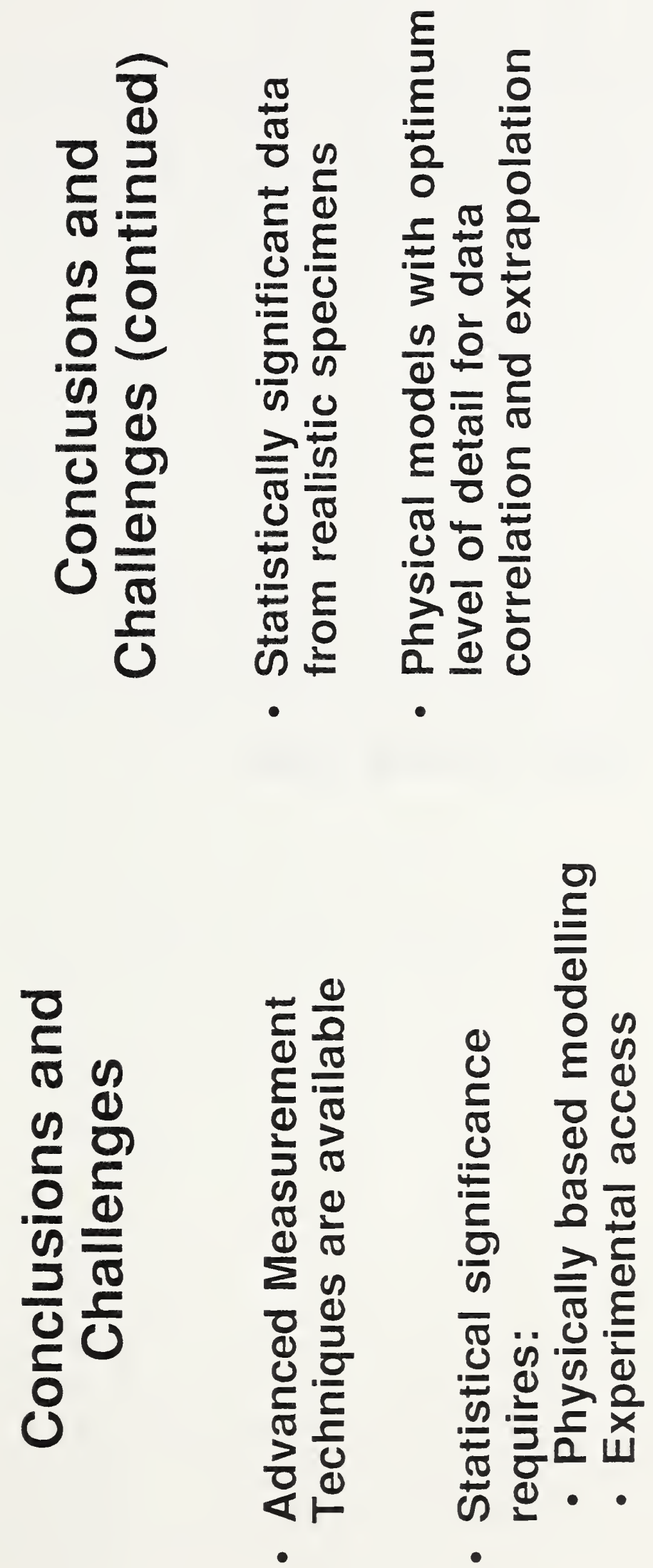


\section{JOHN KELLY, SRC}




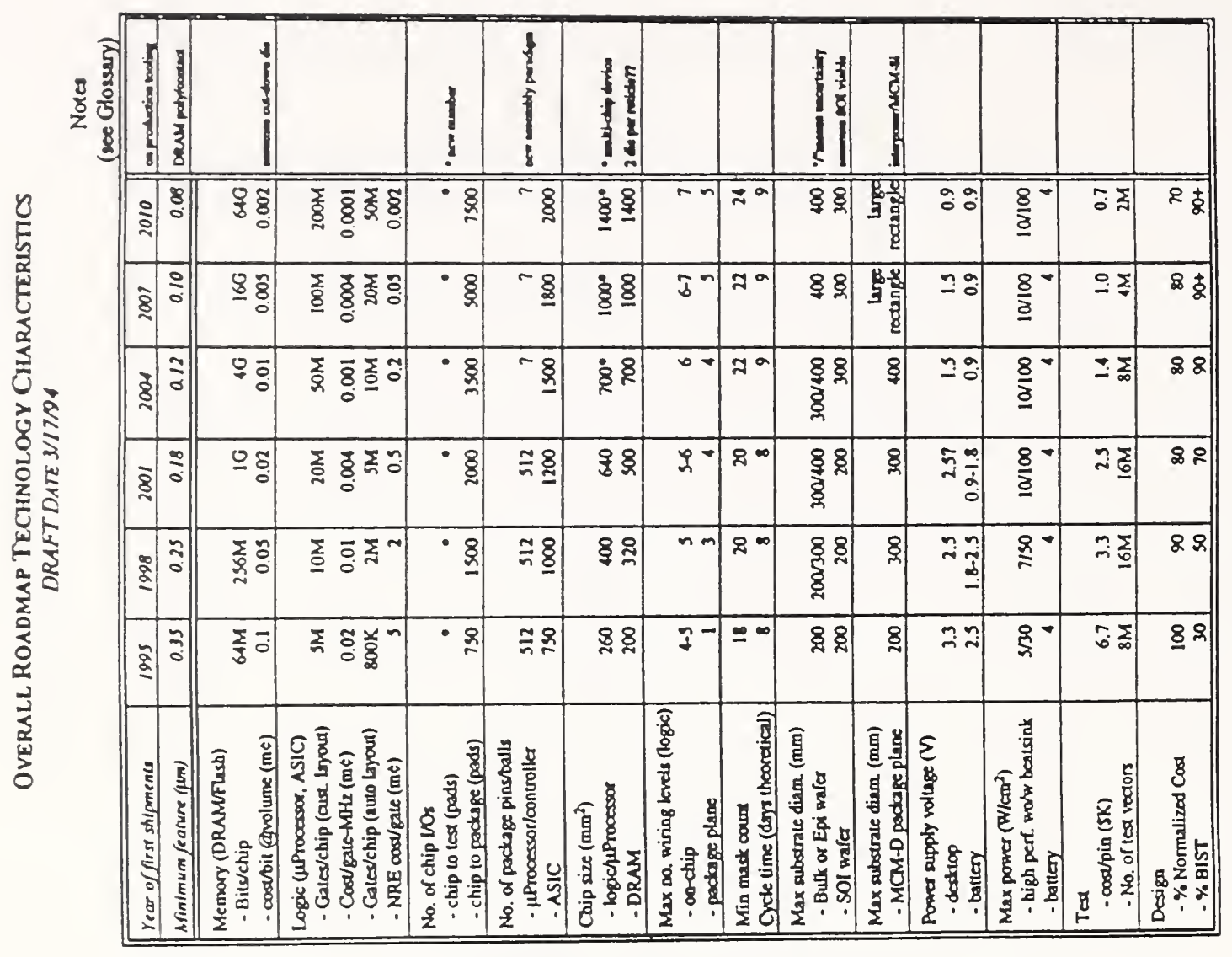

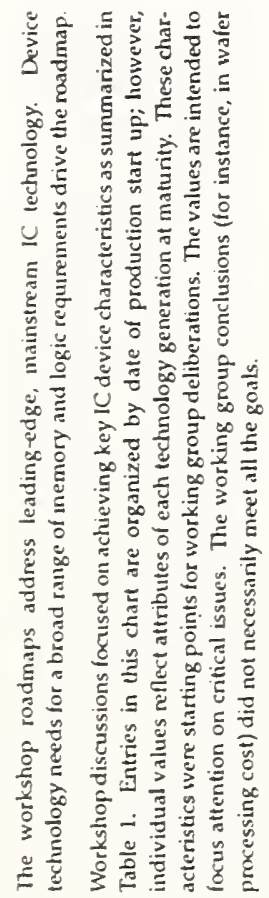

\begin{tabular}{|c|c|c|c|c|c|c|c|c|c|c|c|c|}
\hline ఫ్రి & 웅. & ટ્ટ & 09 & 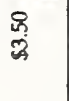 & : & 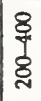 & రั & $\hat{b}$ & $\begin{array}{l}8^{\circ} \\
\vdots \\
\vdots\end{array}$ & 29 & 总 & ర్గి \\
\hline ర్ల & $\cong$ & $\Xi$ & Q & 足 & ర్తి & $\begin{array}{l}8 \\
\text { + } \\
\\
\end{array}$ & $\delta_{0}$ & 0 & $\begin{array}{l}\mathbb{\pi}^{*} \\
\frac{\sigma}{\sigma}\end{array}$ & 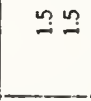 & 8 & 잉욤 \\
\hline $\bar{z}$ & $\stackrel{\infty}{0}$ & $\Sigma$ & $\underline{0} \underset{0}{0}$ & $\stackrel{2}{2}$ & 88 & $\begin{array}{l}8 \\
\\
\\
\end{array}$ & $\stackrel{0}{0}$ & is & $q^{\circ}$ & $\widetilde{N} \simeq$ & ర్లి & 용 \\
\hline$\stackrel{\circ}{\Xi}$ & $\stackrel{2}{a}$ & \} $&{\sum_{0.0} \sum_{0}} &{\begin{array}{l}\not \\
2 \\
2\end{array}} &{\text { ళ్రిల్ల }} &{\begin{array}{l}8 \\
+ \\
\\
\\
\end{array}} &{8} &{\text { in }} &{b^{-}} &{\text {ส }} &{8} &{\underline{0}} \\
{\hline \stackrel{2}{\circ}} &{\begin{array}{c}3 \\
0 \\
0\end{array}} &{\text { 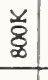 }} &{\text { 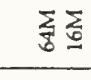 }} &{\begin{array}{l}8 \\
0 \\
\end{array}} &{88} &{\text { \& }} &{\text { o }} &{q} &{\check{\simeq}} &{\text { MN }} &{\text { 负 }} &{\text { \&్లి }} \\
{\hline \tilde{\Xi}} &{\because} &{\text { है }} &{\sum_{0} \sum_{0}} &{\begin{array}{l}8 \\
\end{array}} &{\tilde{y} \tilde{\beth}} &{\text { \&্ন }} &{\overline{0}} &{m} &{\Omega^{m}} &{\stackrel{n m}{m}} &{8} &{8:} \\
{\hline} &{\text { 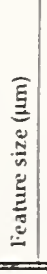 }} &{\frac{a}{\tilde{g}}} &{\text { 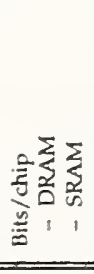 }} &{\text { 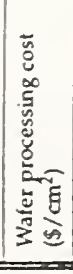 }} &{\text { 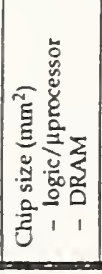 }} &{\text { 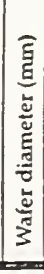 }} &{\text { 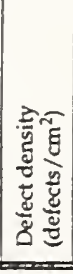 }} &{\text { 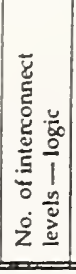 }} &{\text { 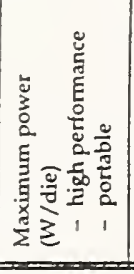 }} &{\text { 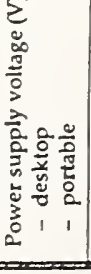 }} &{\begin{array}{l|l} & \\
\vdots \\
\vdots \\
\vdots \\
\vdots \\
\vdots \\
2\end{array}} &{\text { 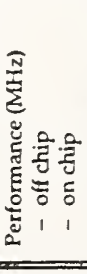 }} \\
$\hline
\end{tabular}



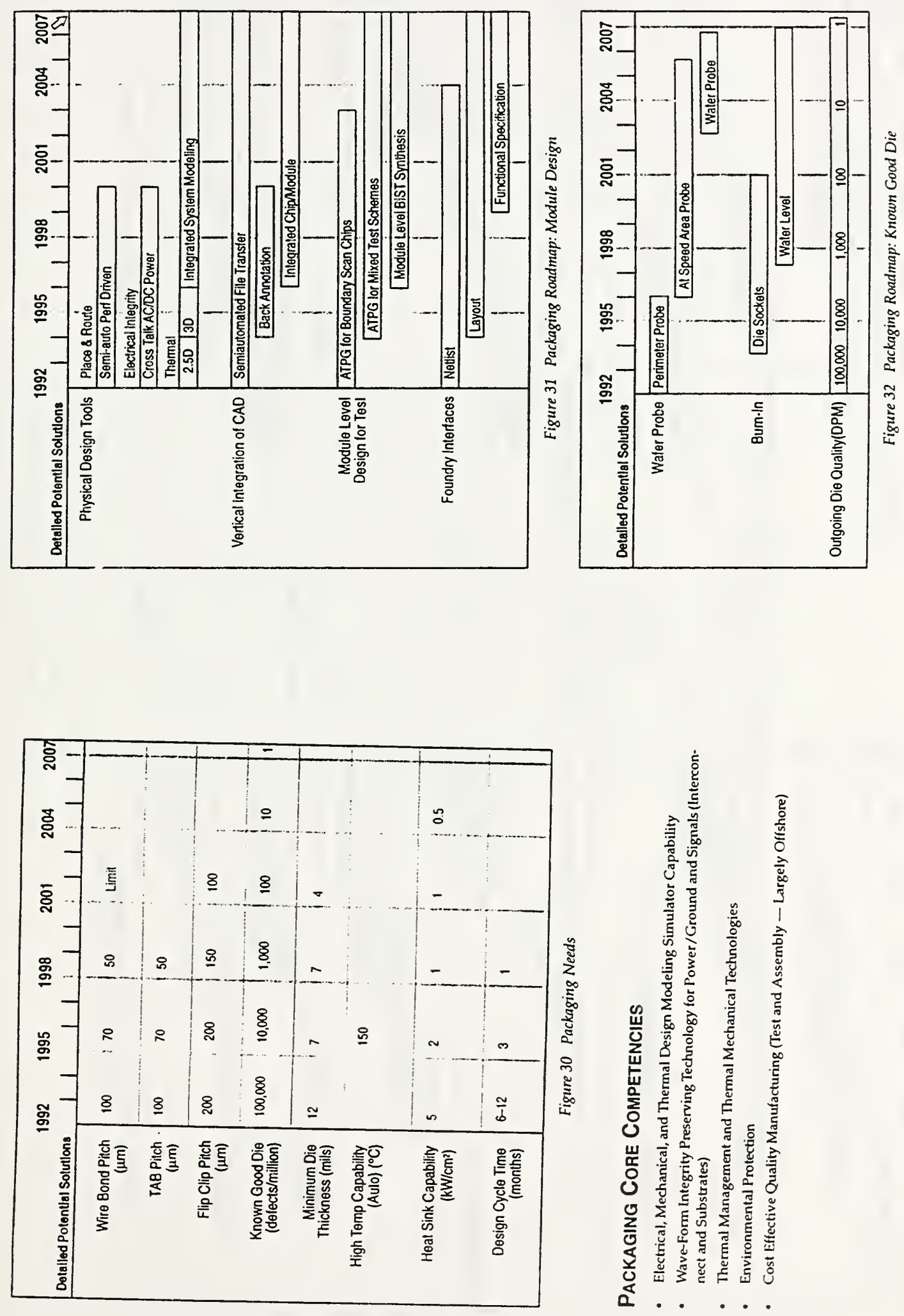

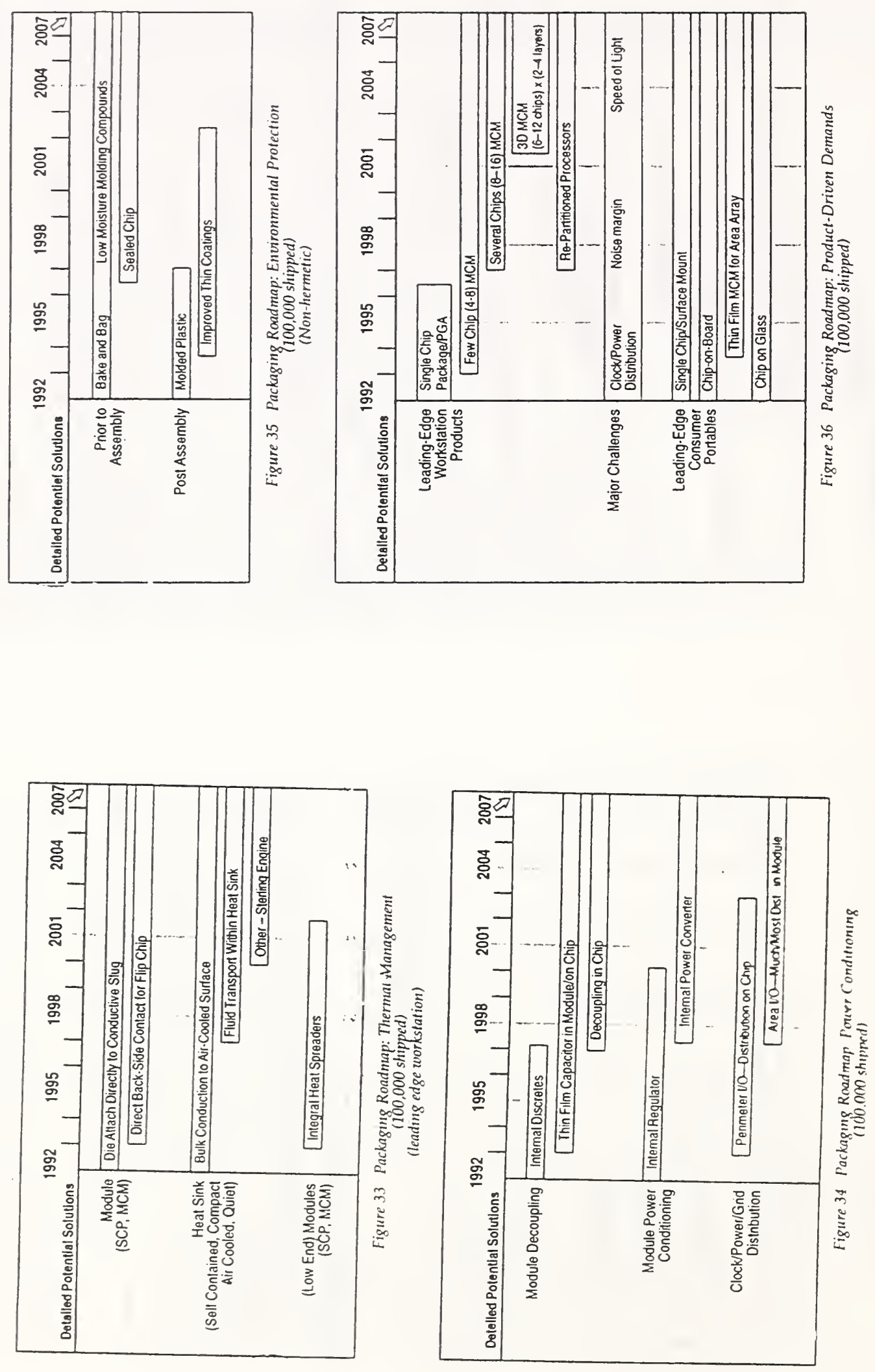


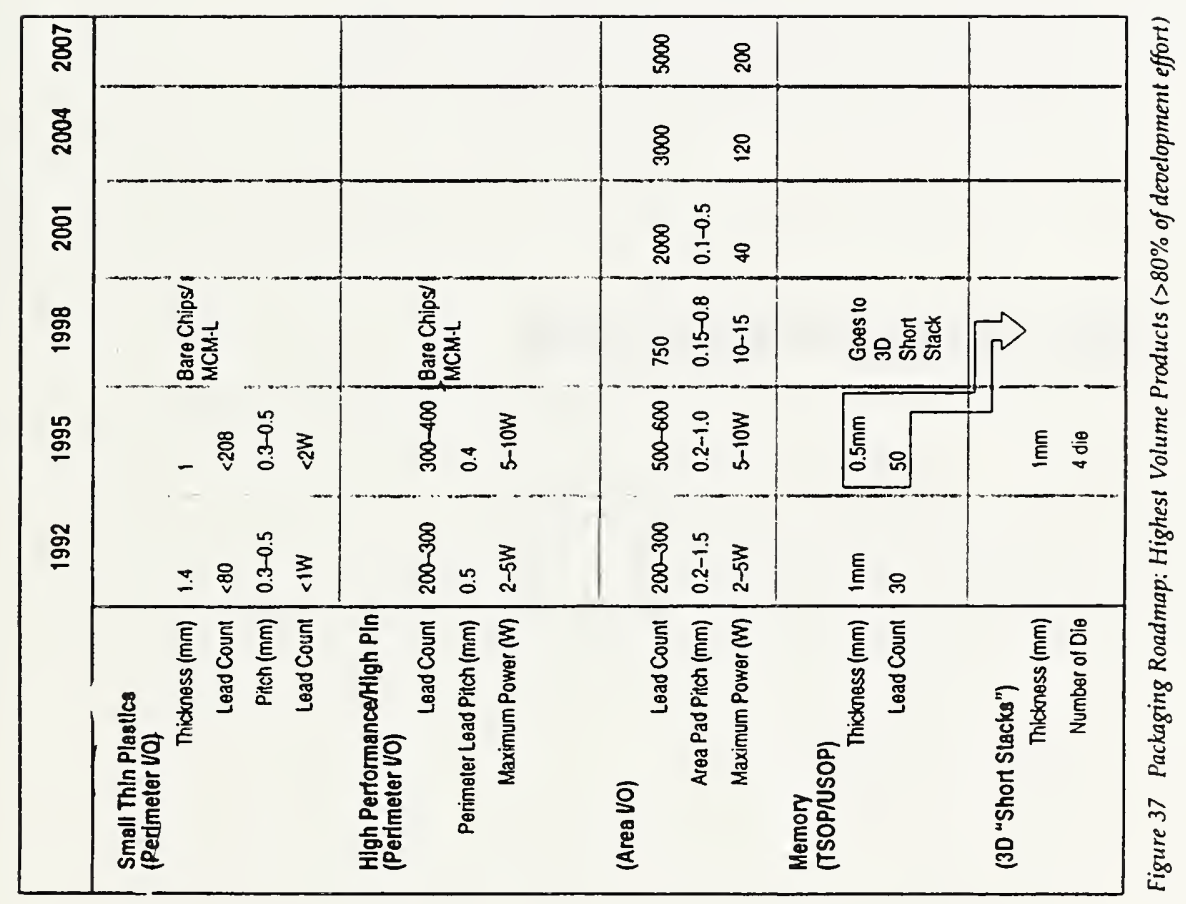


DaVid Bergman, IPC 


\section{PROGRAMS}

- SEMINARS

- STATISTICAL SERVICES

- MARKETING DATA

- STANDARDSISPECIFICATIONS

- TECHNICAL REPORTS

- HOW'S BUSINESS SURVEYS

- TESTING PROGRAMS

- WORKSHOPITRAINING

- PROFICIENCY CERTIFICATION

- RESEARCH

\section{PROGRAM REQUIREMENTS}

- CONSENSUS

- DIRECTION

- FOCUS

- SCHEDULING

- FLEXIBILITY

- VISION

- organizational STRUCTURE 


\section{IPC Present Status}

680 Regular Members

$570 \quad$ Allied Members

415 Associate Members

115 Technical Liason Members

105 Government Members

1885 TOTAL

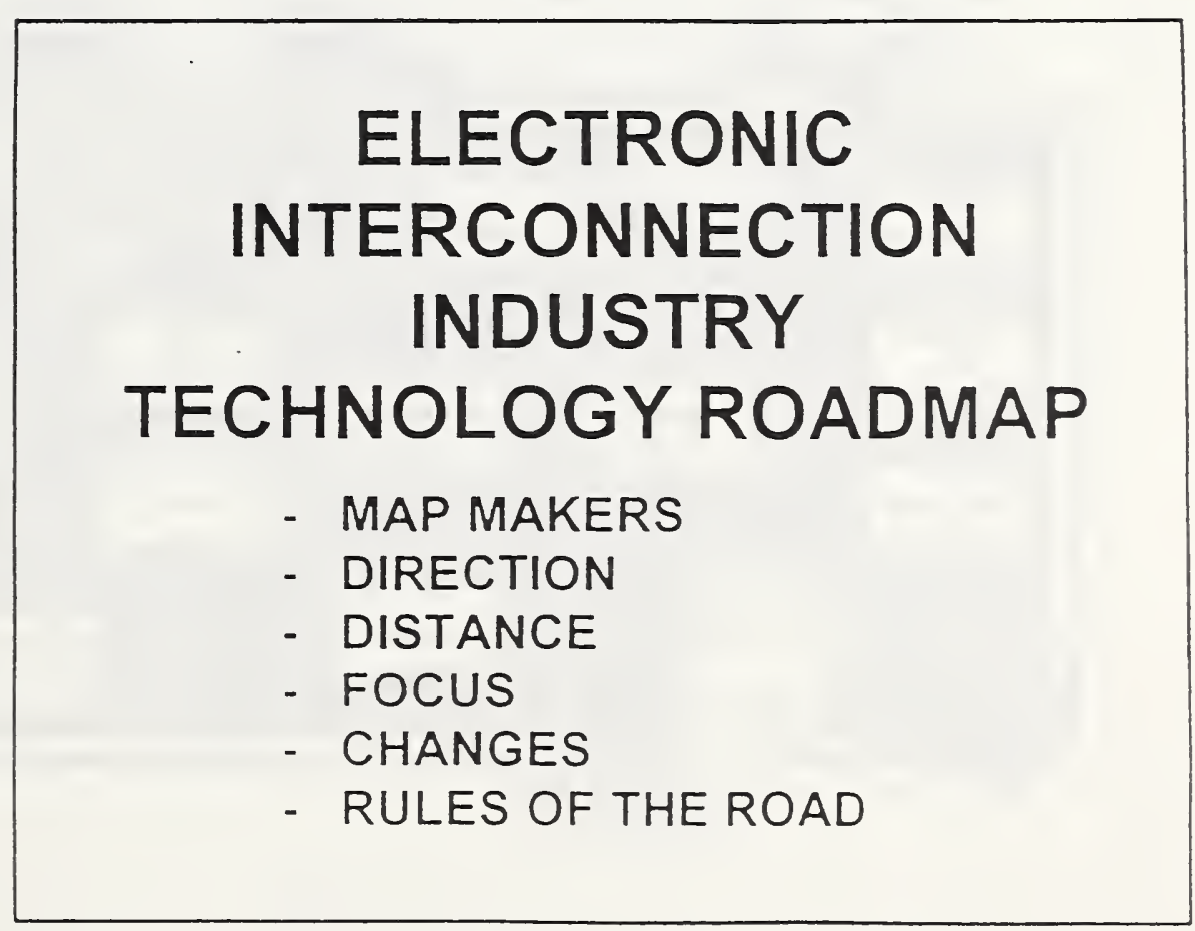




\section{TECHNOLOGY ROADMAP}

INCLUDES ALL MEMBERS OF THE SUPPLY CHAIN FOR VIEWS ON COMPONENTS, OEM MANUFACTURING ISSUES, MATERIALS, INDUSTRY TOOLS AND MANUFACTURING SERVICES

METHODOLOGY BASED ON:

$\checkmark$ COOPERATION

- COORDINATION

- COMMUNICATION

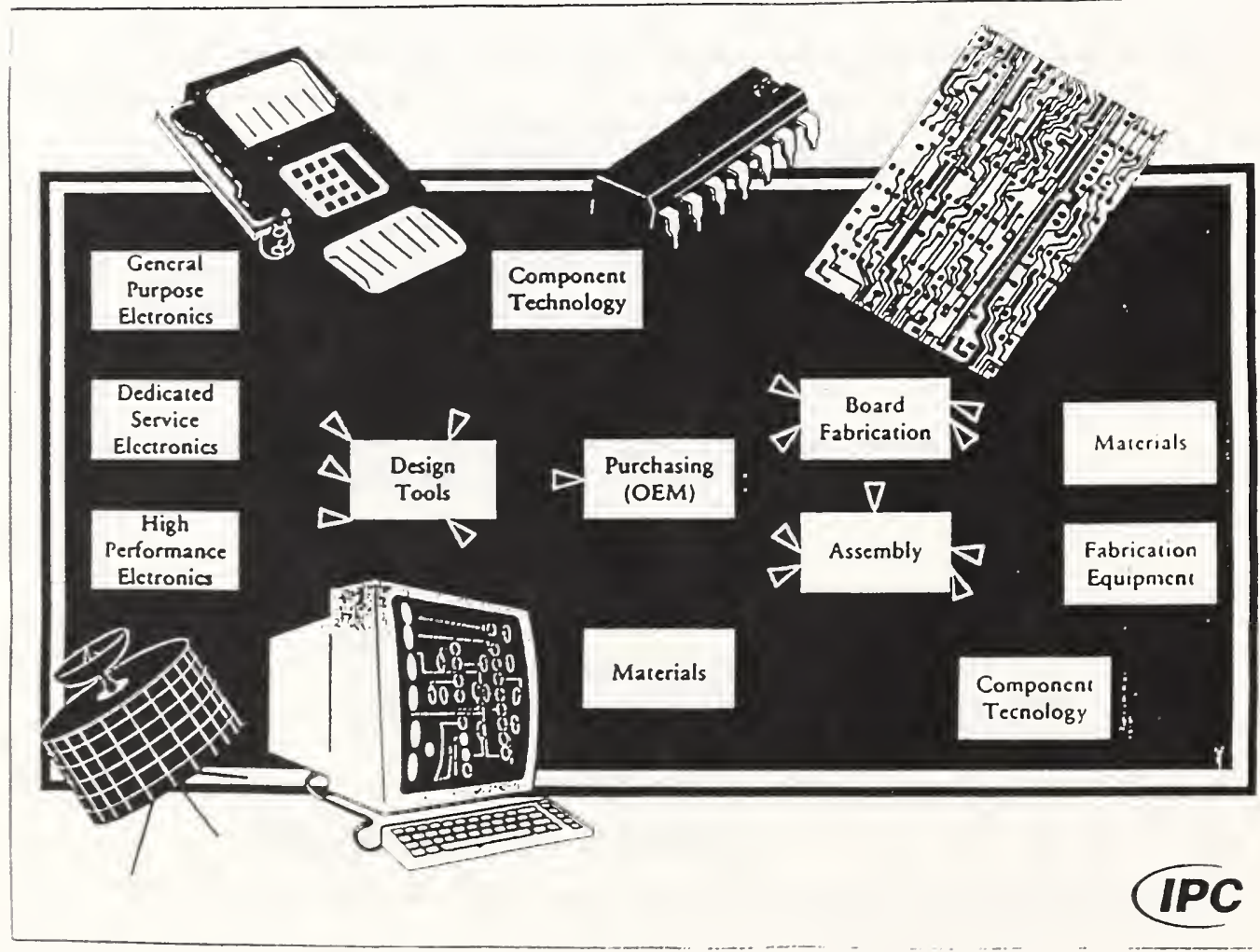




\section{MEMBERSHIP SURVEY}

C - COMPONENT PACKAGING

G - GENERAL ELECTRONICS

D - DEDICATED SERVICE

H - HIGH PERFORMANCE

T - DESIGN TOOLS

M - MATERIALS

F - FABRICATION

A - ASSEMBLY

$P$ - PURCHASING

THIS SURVEY WILL PROVIDE RESOURCE MATERIAL FOR DEVELOPING THE 1994 TECHNOLOGY ROADMAP OF SHORT TERM, ANO LONG TERM REOUIREMENTS OF THE ELECTRONIC INTERCONNECTION ANO ASSEMBLY INOUSTRY. YOUR PARTICIPATION IN THIS SURVEY IS PROBABLY THE MOST IMPORTANT CONTRIBUTION THAT YOU MAKE FOR YOUR COMPANY ANO THE INDUSTAY IN 1994. IN ORDER TO COMPLETE THIS SURVEY, PLEASE ADORESS ALL OUESTIONS IN WHICH YOU HAVE ANY INTEREST OR CAN PROVIDE AN INPUT.

PLEASE PROVIDE YOUR MOST IMAGINATNE, INNOVATIVE, EVEN "BLUE-SKY" INPUT TO THE SURVEY.

WE WANT YOUR IOEAS FOR TODAY, TOMORROW AND BEYOND REGAROING THE NEEDS OF

- The Original EOUIPMENT MaNufacturer that drives

- THE ELECTRONIC INTERCONNECTION MANUfACtURERS that drives

- The Suppliers of Matefials and Eouipment

\section{SURVEY}

FOR THE DEVELOPMENT OF THE 1994

ELECTRONIC INTERCONNECTION INDUSTRY

TECHNOLOGY ROADMAP 


\section{Preliminary Survey}

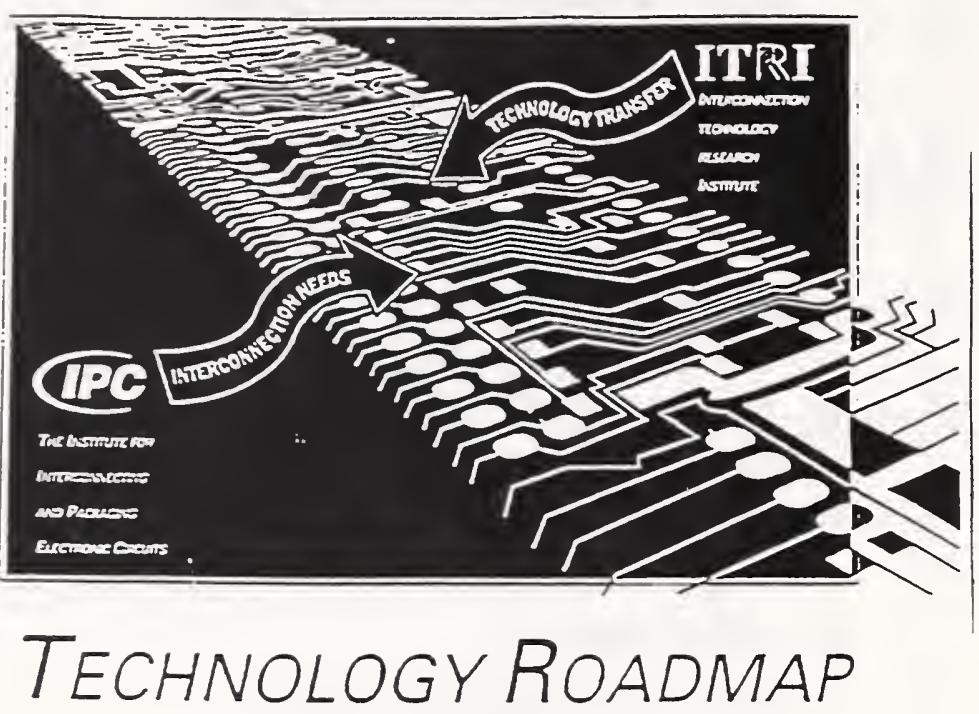

THE FUTURE

OF THE ELECTRONIC

I.VTERCONNECTION INDL'STRY

\section{MARCH 1994}

\section{RESPONDENTS}

\section{OVER 600 \\ RECOMMENDATIONS FOR}

$$
1993 \text { - } 1995 \text { - } 1997
$$

- TYPE OF RESPONDENT FUNCTIONS

- MANAGEMENT

- ENGINEERING

- PRODUCTION

- PURCHASING

- QUALITY

- PROCESS CONTROL

- TECHNICAL LIAISON

- VENDOR CONTROL

- RESEARCH 


\section{SEVEN ISSUES PER TOPIC}

\section{EABRICATION EXAMPLE}

F1 INNERLAYER LINE WIDTH AND SPACE

F2 BOARD SIZE

F3 BOARD \& INNER CORE THICKNESS

F4 HOLE SIZES (THROUGH, BLIND, BURIED)

F5 NUMBER OF LAYERS

F6 TEST AND EVALUATION

F7 SURFACE CONDITIONS

\section{THE MAP MAKERS}

- TECHNOLOGY ROADMAP WORKSHOP

- 100 EXPERTS MET IN APRIL

- THREE DAY INTENSIVE REVIEW

- TECHNOLOGISTS PRESENTATIONS

- ANALYSIS OF SURVEY REPORT

- DEVELOPED ROADMAP INITIAL DRAFT 


\section{DETAILS OF FIRST ROADMAP DRAFT}

- ALL NINE TOPICS AND THEIR DETAILED BREAKDOWN WERE DEVELOPED BY THE FACILITATORS AND THEIR TEAM MEMBERS

- DISCUSSION

- OUTLOOK

- SUCCESS FACTOR

- SHOWSTOPPERS

- RECOMMENDATIONS

\section{MAJOR SHOW STOPPERS}

- LIMITED METHODOLOGY TO DEVELOP AND INTEGRATE NEW TECHNOLOGY INTO MANUFACTURING

- NO SINGLE SET OF INDUSTRY/ GOVERNMENT/ ECONOMIC PROGRAMS FOR THE SUPPLY CHAIN

- DESIGN TOOLS ARE NOT COORDINATED WITH PRODUCTION PROCESSES

- LACK OF ENVIRONMENTALLY FRIENDLY MATERIALS AND MANUFACTURING PROCESSES

- POOR CUSTOMER/ MANUFACTURING/ SUPPLIER RELATIONSHIPS 


\section{REQUIREMENTS FOR GLOBAL COMPETITIVENESS}

- STRONG ELECTRONICS INDUSTRY

- HEALTHY MEMBERS OF SUPPLY CHAIN

- ELECTRONIC EQUIPMENT DRIVES

- PRINTED BOARD ASSEMBLY DRIVES

- PRINTED BOARD FABRICATION DRIVES

- MATERIAL/ PROCESSING SUPPLIERS

\section{INTERNATIONAL ELECTRONIC EQUIPMENT REQUIRES GREATER:}

- MINIATURIZATION

- COST REDUCTION

- PERFORMANCE

- RELIABILITY (QUALITY IS EXPECTED) 


\section{MAIN CAUSES OF NEEDS TO CHANGE:}

- STIFF AND GROWING COMPETITION (SUBSIDIZED BY LARGE COMPANIES)

- SHIFT IN RESPONSIBILITY FOR RESEARCH AND DEVELOPMENT

- GROWTH AND DEMAND FOR ELECTRONIC PRODUCT PERFORMANCE

\section{PRIORITIZATION MEETING}

- SPECIAL REVIEW OF COMMENTS

- ANALYSIS OF 164 RECOMMENDATIONS

- BREAKDOWN ACTION

- RESEARCH

- NATIONAL INITIATION

- MARKETING

- COMMITTEE PROJECTS 


\section{GROUPING OF RECOMMENDATIONS}

- TECHNOLOGY ASSESSMENT METHODS

- DESIGN TOOLS COMPETENCE

- NEW MATERIALS THAT WITHSTAND STRESS

- IMPROVE QUALITY AND RELIABILITY METHODS

- NEW ATTACHMENT MATERIALS

- CUSTOMER ACCEPTANCE OF NEW IDEAS

- SIMPLIFIED, EFFICIENT MANUFACTURING PROCESSES

- NON VISUAL, NON DESTRUCTIVE INSPECTION METHODS

- ENVIRONMENTALLY FRIENDLY, SAFE MANUFACTURING

\section{PWB FABRICATION SHOWSTOPPERS}

- LAMINATE AND FOIL FINISH

- IMAGING TECHNOLOGY

- TESTING

- AVAILABILITY OF CAPITAL EQUIPMENT

- HANDLING OF MATERIALS IN THE PROCESS

- NUMBERS OF PROCESS STEPS

- ENVIRONMENTAL CONDITIONS

- VERTICAL PARTNERSHIPS

- PROCESS AVAILABILITY 


\section{WORKSHOP DELIBERATION REVIEW}

- TEAM PROJECT DESCRIPTION RECOMMENDATION FINE TUNING

- ORGANIZE PROJECT DESCRIPTIONS INTO FOCUS GROUPS

- MATERIALS

- TOOLS

- FABRICATION

- ASSEMBLY

- OTHER

- TEAM FORMATION

- (WEDNESDAY GROUPING)

- (NEW LIAISONS)

- PROJECT ASSIGNMENT

- (MINIMUM - 6 PROJECT ANALYSIS WRITE-UPS PER TEAM)

\section{IMPLEMENTATION WORKSHOP STRATEGY}

- RESOURCE MATERIAL 1993

- SUMMARY OF 1994 SURVEY

- BREAK INTO REAFFIRMATION TEAMS

- (2) DESIGN TOOLS

- (2) MATERIALS

- (3) FABRICATION

- (2) ASSEMBLY

- GOAL

- ANALYZE RESOURCE DATA AND 1994 SURVEY RESULTS AND REAFFIRM, UPDATE, ENHANCE RECOMMENDATIONS 


\section{ACHIEVING \\ COMPETITIVE EXCELLENCE}

CHANGE THE WAY WE:

- MANUFACTURE PRODUCTS

- INTERACT WITH THE ENVIRONMENT

- TRANSFER DATA

- SERVICE THE CUSTOMER

- DEVELOP NEW TECHNOLOGY

- PURCHASE PRODUCTS

\section{RECOMMENDATIONS FOR ACTION}

- METHODS FOR ESTABLISHING KNOWN GOOD DIE

- FLUXLESS SYSTEMS AND PROCESSES FOR ATTACHMENT

- CORRELATION OF NOBLE METAL EFFECT ON EMBRITTLEMENT AND SOLDERABILITY

- REDUCTION OF COMPONENT PACKAGING COSTS THROUGH THE USE OF BULK FEED MECHANISMS

- REPAIR METHODS FOR FINE PITCH COMPONENTS

- HIGH YIELD PRODUCTION EQUIPMENT FOR EASE OF HANDLING BARE CHIPS AND FLIP CHIP APPLICATIONS

- CONDUCTIVE ADHESIVES (PHOTOIMAGABLE) 


\section{WORKSHOP DELIVERABLES}

- PRESENTATION OF SUGGESTED RESEARCH BY TEAM LEADERS

- INPUT FOR PUBLISHING EXECUTIVE OVERVIEW OF 1994 ROADMAP

- COMMITMENTIINTEREST LEVEL IN FORMATION OF COOPERATIVE RESEARCH TEAMS

- COMMITMENT TO PARTICIPATE IN ITRI

- PUBLICIZE INDUSTRY PROACTIVE POSITION

\section{Rigid Printed Board Substrate Materials}

- Develop core material less than $\mathbf{0 . 0 0 2}$ inch thick

- Develop clad laminate with smooth surfaces with fine conductors

- Form consortia to correlate UL 4-point oven aging with other methods

- Develop techniques to minimize environmental impact of producing base materials

- Develop materials which reduce hole formation costs

- Develop relationship between plated throughhole failure and measurable physical properties of rigid laminate 


\section{Conductors}

- Lack of characterization and measurement methods for thin foils, as well as lack of thin foil handling techniques are serious concerns

\section{Dielectric and Electrical Properties}

- Develop low Dielectric Constant (DK) substrates

\section{Component Attachment Materials}

- No proven alloy alternatives for tin-lead solders

- Lead-free solders may be driven by environmental and political concerns rather than technical reasons

- Conductive adhesives have the following problems:

- Moisture absorption which impacts adhesion

- Poor mechanical shock properties
- Poor high frequency $(10 \mathrm{mHz})$ performance

- Undesirable high temperature properties 
Board and Inner Board (Core) Thickness

- Establish techniques and equipment for handling thin materials

Surface Conditions/Coatings

- Eliminate metallic coatings by promoting use of bare copper with protective coatings and encourage $R \& D$ for compatible fluxes and reliable

Fine Pitch Technology

- Correlate effects of noble metals on surface quality, embrittlement and solderability

Attachment Techniques

- Develop fluxless systems and process

- Develop conductive adhesive applications and photo-imagable conductive adhesives 


\section{Davis Hartman, Motorola, OIDA}




\title{
OIDA TECHNOLOGY ROADMAP PROGRAM
}

\author{
DAVIS HARTMAN
}

MOTOROLA

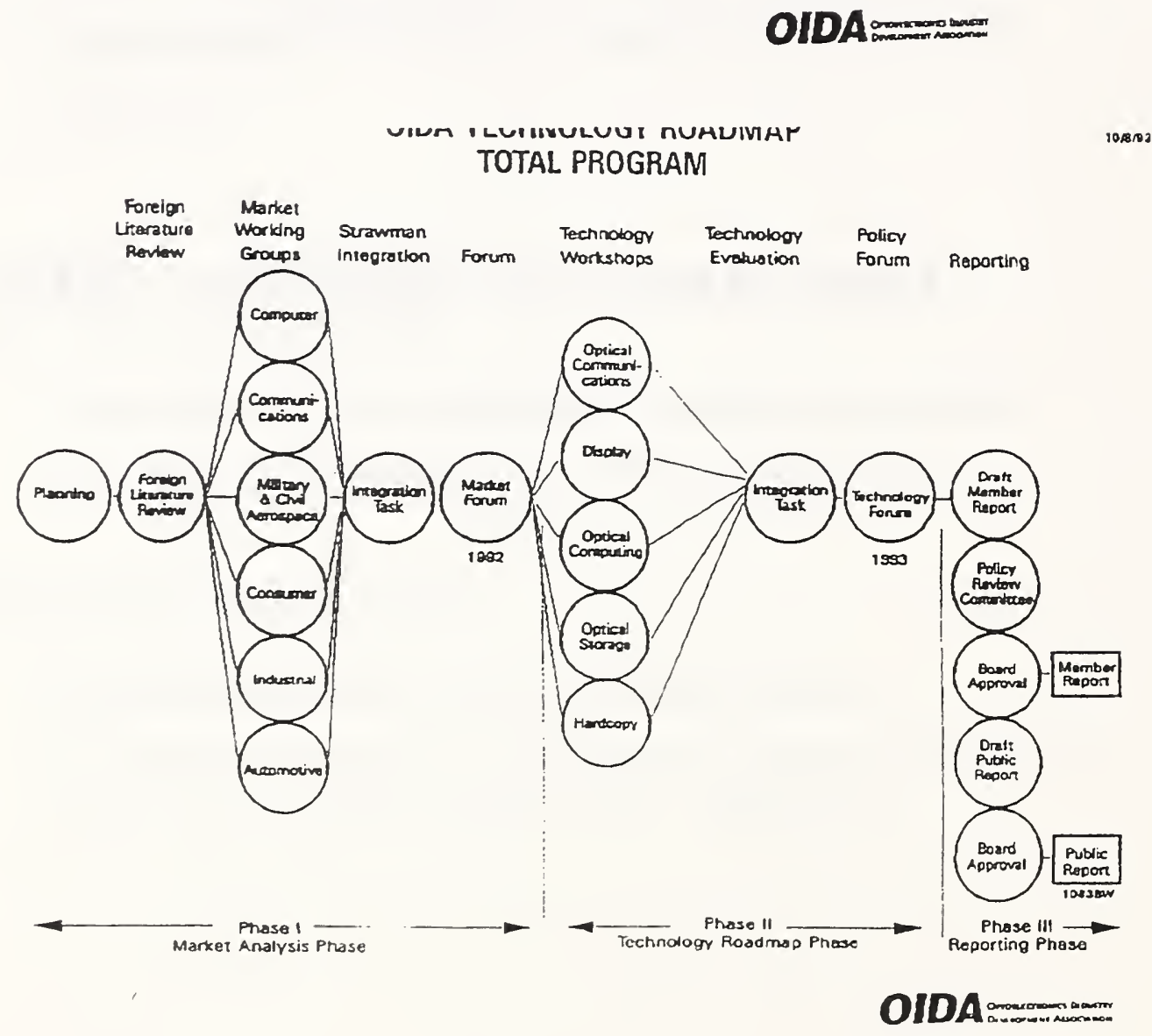




\section{OIDA TECHNOLOGY WORKSHOPS - PHASE II ATTENDANCE SUMMARY}

\begin{tabular}{|c|c|c|c|c|}
\hline & Industry & Academic & Gov't & Total \\
\hline Display & 44 & 5 & 9 & 58 \\
\hline Optical Communications & 55 & 5 & 4 & 64 \\
\hline Optical Storage & 16 & 11 & 4 & 31 \\
\hline $\begin{array}{l}\text { Optics In Swltching } \\
\text { \& Computing }\end{array}$ & 19 & 18 & 8 & 45 \\
\hline \multirow[t]{2}{*}{ Hardcopy } & $\underline{26}$ & 3 & 1 & 30 \\
\hline & 160 & 42 & 26 & 228 \\
\hline
\end{tabular}

OIDA TECHNOLOGY ROADMAP BREAK-OUT DISCUSSION GROUPS

\begin{tabular}{|c|c|c|c|c|}
\hline Display & $\begin{array}{c}\text { Optlcal } \\
\text { Communications }\end{array}$ & Optice LStorage & $\begin{array}{l}\text { Optles in } \\
\text { Switching \& } \\
\text { Computing }\end{array}$ & Hardcopy \\
\hline $\begin{array}{l}\text { A. Passive } \\
\text { Matrix LCD } \\
\text { B. Active } \\
\text { Matrix LCD } \\
\text { C. Electro- } \\
\text { luminescent } \\
\text { O. Plasma } \\
\text { E. Field } \\
\text { Emission } \\
\text { F. LED } \\
\text { G. 3D/Holography } \\
\text { H. PrOjoctlon } \\
\text { L. CRT }\end{array}$ & $\begin{array}{l}\text { A. Telecom } \\
\text { B. Datacom } \\
\text { C. Package } \\
\text { Design } \\
\text { and Mifg. } \\
\text { D. Active } \\
\text { Semiconductor } \\
\text { Devices } \\
\text { E. Passive } \\
\text { Devices } \\
\text { F. Fiber and } \\
\text { Active Flber } \\
\text { Devices }\end{array}$ & $\begin{array}{l}\text { A. Media } \\
\text { B. Lasers and } \\
\text { Optical Heads } \\
\text { C. Noncon- } \\
\text { yentional } \\
\text { Optical } \\
\text { Storage } \\
\text { D. Appication- } \\
\text { Driven } \\
\text { Technology } \\
\text { Requirements }\end{array}$ & $\begin{array}{l}\text { A. Photonics in } \\
\text { Switching } \\
\text { B. Intramachine } \\
\text { Intorconnection } \\
\text { C. Signal, Neural, } \\
\text { Imago, and } \\
\text { Miultimenslonal } \\
\text { Processing } \\
\text { D. Optlcal Memory } \\
\text { Access and Data } \\
\text { Base Systems }\end{array}$ & $\begin{array}{l}\text { A. Printers - } \\
\text { Electro- } \\
\text { photogrephio } \\
\text { B. Printers - } \\
\text { inkjot } \\
\text { C. Printers - } \\
\text { ThermaV } \\
\text { Photo- } \\
\text { sensiture } \\
\text { D. Modia } \\
\text { E. Scanners } \\
\text { F. Solld } \\
\text { Imaging }\end{array}$ \\
\hline
\end{tabular}




\section{Téennical/Manufacturing Barriers for Flat Panel Displays}

$\therefore$ PMLCD

- Limited contrast, color saturation, response time, resolation

$\therefore$ AMLCD

- Complex, high-cost processing

* Electroluminescent

- Poor gray scale capability, high cost ICs, limited color

× Plasma

- Poor gray scale, low Iuminous efficiency, high-cost ICs

$\therefore$ Field emission

- Difficult spacer fabrcation and vacurum assembly; needs development of cathode technology

OIDA

\section{DISPLAY TECHNOLOGY ROADMAP \\ Field Emission}

\begin{tabular}{|c|c|c|c|c|c|}
\hline & 1993 & 1990 & 2003 & 2000 & 2013 \\
\hline \multicolumn{6}{|l|}{ PARAMETE? } \\
\hline Escon iesolution & $240 \times 320$ & $1280 \times 1024$ & $1280 \times 1024$ & FETV & MON \\
\hline 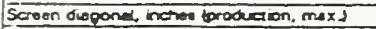 & - & 10 & 20 & 40 & 70 \\
\hline Pexer roscotriton, inos por then & 50 & 170 & 240 & 240 & 240 \\
\hline Eutr reotio & 240 & 1024 & 1024 & 7100 & 1100 \\
\hline Whros luminence, colm2 & 50 & 160 & 250 & 600 & 500 \\
\hline Combor rasio of mbien ithum. & 5 & 10 & 15 & 20 & 20 \\
\hline Gay leved OC9 & 32 & as & os & 128 & 258 \\
\hline Fumber of colors & $\theta$ & $262 \pi$ & $252 \pi$ & AM & $10 \mathrm{~N}$ \\
\hline \multicolumn{6}{|l|}{ Moming andis, +1-dogrees } \\
\hline Horrzantel Q CA > 10.1 & 80 & 80 & 80 & 80 & 80 \\
\hline Vortica e CR $>10: 1$ & 80 & 80 & 80 & 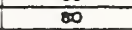 & $\infty$ \\
\hline Ponel rosoches time, max & $<16$ & $<10$ & 518 & $<18$ & $<16$ \\
\hline 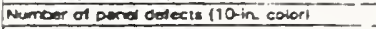 & 0 & 0 & 0 & 0 & 0 \\
\hline 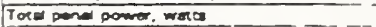 & 5 & 2 & 10 & 100 & 200 \\
\hline \multirow[t]{2}{*}{ anco. 1} & $\because$ & 400 & 300 & 300 & 6000 \\
\hline & & & & & \\
\hline \multicolumn{6}{|l|}{ TECPNOLOFY DEVELOPMEMTS } \\
\hline Crinode menut acterins & - & 10 incte & 20 incties & 40 hathen & 70 inches \\
\hline Speoser: & $200 \mathrm{~V}$ & $500 \mathrm{~V}$ & $2,000 \mathrm{~V}$ & $2.000 \mathrm{~V}$ & $2,000 \mathrm{~V}$ \\
\hline Low ralepe phosption & 1 lumentwath & B hurnerentwot & 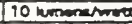 & 12 wrmeralwat & 14 hrmerasmert \\
\hline Vecuarn notegrivloperning lite & 10,000 nourt & 20.000 hour & 40,000 hours & 00,000 haw & 80.000 how \\
\hline Cost-Altocive compusar coriens & - & $1024 \times 1280$ & 204800560 & & \\
\hline \multirow[t]{2}{*}{ cost diecture nidoo } & & & मूTV & PEON & \\
\hline & & & $30-40$ indies & $40-50$ inction & \\
\hline
\end{tabular}




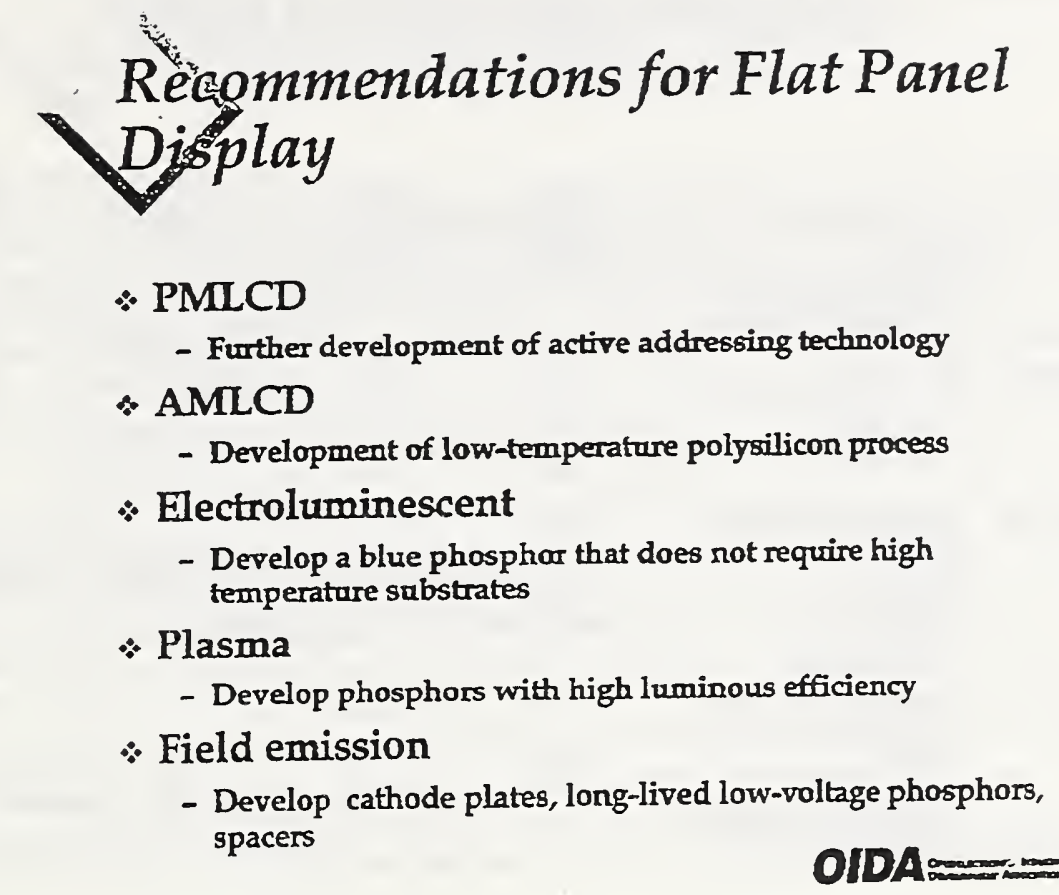

Technical/Manufacturing Barriers
Optical Communications

$\therefore$ Cost of packaging and alignment of active and passive components

$\therefore$ Cost of fiber management

- Overconstrained requirements on device/package design

* Modeling/simulation/CAD tools

- Common approaches/standards in several areas, e.g. high-speed datacom

* Concurrent engineering (interaction between device and package design)

OIDA 
TELECOMMUNICATONS TECHNOLOGY ROADMAP

Local Loop/CATV Alternatives/fiber to the Curb

\begin{tabular}{|c|c|c|c|c|c|c|}
\hline & & 1390 & 1898 & 2003 & 2008 & 2013 \\
\hline \multirow[t]{5}{*}{ GDER } & 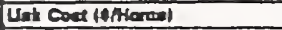 & 160 & 75 & 40 & 25 & 15 \\
\hline & Finer & SNGIES & Sol Glass & Su Gbes & SMGles & SM Gou \\
\hline & mederin. ATs & & 100 & $E 0$ & 25 & 12.5 \\
\hline & 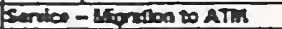 & F & & & & \\
\hline & 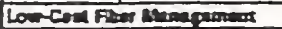 & & F & & & \\
\hline \multirow[t]{5}{*}{ 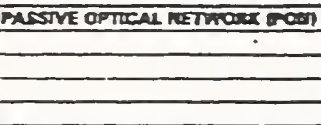 } & une and & 20 & TE5 & 165 & 022 & 022 \\
\hline & Subeoser End Dugdex Tramo & & & & & \\
\hline & 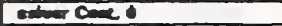 & $\infty$ & 100 & 50 & 20 & 10 \\
\hline & Speorex Ind & Gen Fond & Geof Fined & Porye & Pohmes & Potyiner \\
\hline & $\cos 1$ & 40 & 20 & 10 & 5 & 3 \\
\hline & & का & 100 & 50 & 610 & \\
\hline \multirow[t]{6}{*}{ 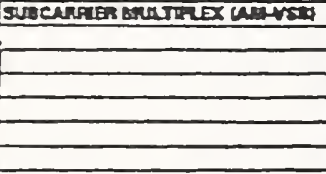 } & 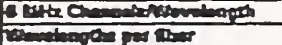 & $\frac{60}{3}$ & $\frac{100}{4}$ & $\frac{320}{4}$ & $\frac{640}{8}$ & $\frac{840}{18}$ \\
\hline & ondingen & Dinet & Diact of Extord & Drece of Exomd & Divet of Botint & Dree or Eumern \\
\hline & Findencos & $1200=0$ & 1300 1 $1950 \mathrm{now}$ & $1300.1600 \mathrm{~nm}$ & $13001600 \mathrm{~mm}$ & $1500.1550 \mathrm{~mm}$ \\
\hline & 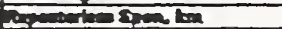 & 15 & 18 & 18 & 15 & 15 \\
\hline & An & & & & & \\
\hline & 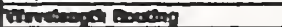 & & & & E & \\
\hline & & & & & & \\
\hline \multirow[t]{7}{*}{ 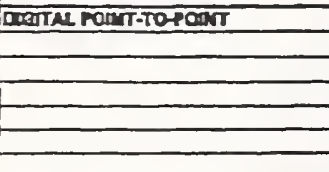 } & 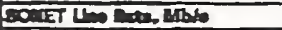 & 3ED & 822 & $\underline{m 2}$ & 022 & \\
\hline & 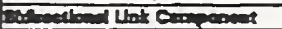 & & & & & \\
\hline & con & 2500 & 200 & 180 & 100 & \\
\hline & 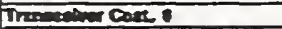 & 75 & 58 & 10 & 9 & \\
\hline & 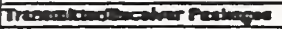 & & & Uacondedan-Cont & celems & \\
\hline & 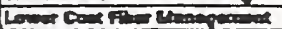 & & & & & \\
\hline & ad consenders & & & & & \\
\hline
\end{tabular}

\section{Récommendations for Optical Communications}

$\therefore$ Transmitters and receivers

- New packaging approaches with lower cost materials; automated alignment; high-yield processes for device fab

* Connectors

- New low-cost materials, e.g. polymers and polymex/ceramic composites; less labor-intensive field installation methods

$\therefore$ Fiber/cable management

- Improved design for fiber cable and fiber and cable management apparatas; new materials and tools

$\therefore$ Couplers, splitters and WDMs

- Low-cost packaging and alignment approaches; elimination of pigtailing 


\section{TêsnicalManufacturing Barriers for Optical Storage}

- Testing and measurement capability for magneto-optic media at high storage densities

* Molding and mastering of high-capacity substrates

* Low jitter noise media for pulse-width modulation

- Short wavelength lasers

- Integration of optical heads

OIDA

OPTICAL STORAGE TECHNOLOGY ROADMAP

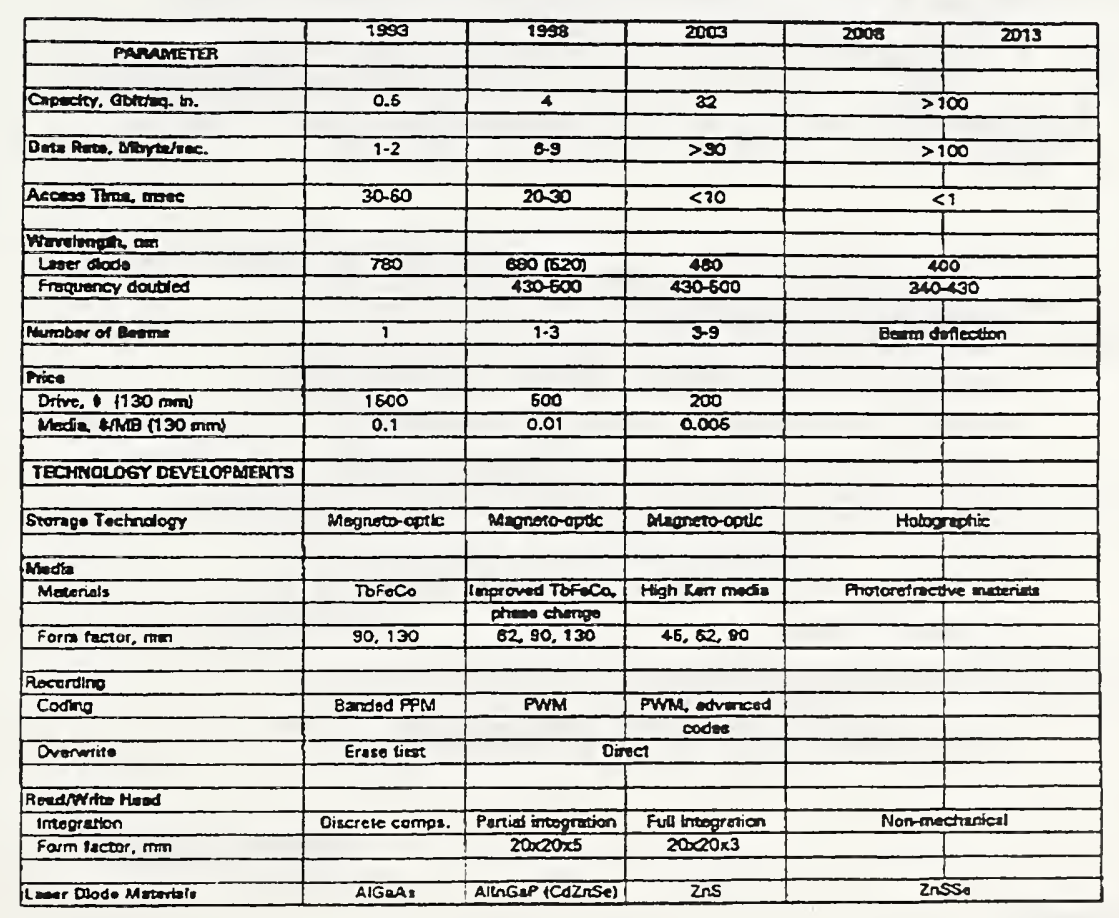




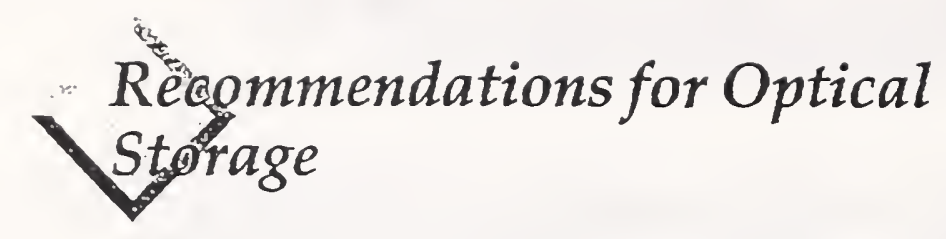

- Short wavelength sources

- Central facility for advanced engineering samples; address manufacturing scale-up issues

\section{* Advanced media}

- Test center for evaluation of media under realistic conditions; new plastic substrate technology

$\therefore$ Advanced optial storage systems

- Support for interdiscplinary programs involving applications/software/technology

* 3-D optical memories

- Accelerake development of prototype recording systems; sapport 3-D materials R\&D

\section{OIDA}

\section{Teehnical/Manufacturing Barriers fo. Hardcopy Technologies}

$\therefore$ Simplified, single-pass process for color electrophotography

$\therefore$ Low-cost high-power laser diodes, more sensitive media for thermal printing

$\because$ Post-exposure processing for photosensitive media

- Common color standards for scanning and reproduction

* Lower cost UV sources, improved photopolymers for solid imaging 
HARDCOPY TECHNOLOGY ROADMAP

Therma/Photosensitive

\begin{tabular}{|c|c|c|c|c|c|}
\hline & 1903 & दर्दु8 & 2003 & & 2013 \\
\hline \multicolumn{6}{|l|}{ FARAMETER } \\
\hline Imego Quetity. dDi & 300000 & $000 / 60$ & $8 \infty \pi 00$ & $\infty$ & कल/1200 \\
\hline Line per recection, w/mm & $\frac{60}{60}$ & $\frac{12 \sqrt{2}}{12 \sqrt{2}}$ & $\frac{1216}{1216}$ & $\sqrt{220}$ & 1226 \\
\hline Oms (reflectlva) & $2 . e \sqrt{2.8}$ & 2.8212 & 25150 & 1020 & 3.250 \\
\hline Omsx tromanitsive) & 2.23 .6 & $2.6 \sqrt{26}$ & $28 / 25$ & $26 \sqrt{2.7}$ & 2.61 .7 \\
\hline Dinin (rotherthe) & 0.030 .06 & 0.020 .05 & 0.020 .04 & 0.020 .00 & 0.020 .02 \\
\hline काn (roventome & 0.0110 .06 & 0.01004 & 0.0110 .02 & 0.010 .02 & 0.0110 .02 \\
\hline 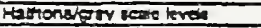 & \multicolumn{2}{|c|}{ 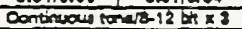 } & & & $\Rightarrow$ \\
\hline \multicolumn{6}{|l|}{ Soeed the mTe $8 \times 10$} \\
\hline Time to firs phin in & $6-10$ & 1 & 0.8 & 0,25 & 0.25 \\
\hline Trow & $\frac{10}{28}$ & $<1$ & $\angle 0.5$ & Q1 & 0.1 \\
\hline Wred cos p000 18210 & 2,10100 & $2 \sqrt{20}$ & $1 / 190$ & 0.515 & 0.23 \\
\hline Cor per per i $18 \times 101$ & 2.501 .40 & $0.00 \% .70$ & $0.20 \% 0.60$ & 0.100 .50 & 0.000 .40 \\
\hline (8:20 & 0,0000 & $D+\sin x$ & Pones & Breter & Dind \\
\hline 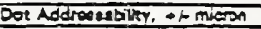 & 40000 & 200 & $20 / 15$ & 2012 & $20 \pi 0$ \\
\hline \multirow{2}{*}{\multicolumn{6}{|c|}{ 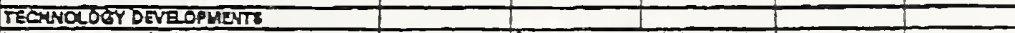 }} \\
\hline & & & & & \\
\hline \multirow[t]{2}{*}{ Low-oos themel printer } & & \multirow{2}{*}{\multicolumn{2}{|c|}{ 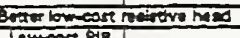 }} & & \\
\hline & & & \multirow{2}{*}{\multicolumn{2}{|c|}{ Hechoore, if }} & \\
\hline \multirow{2}{*}{ Mgh qualing themen perier } & & & & & \\
\hline & & & 100 & 60 & 25 \\
\hline \multirow{3}{*}{ Silver harise xanter } & \multirow{2}{*}{\multicolumn{2}{|c|}{ 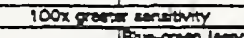 }} & & & \\
\hline & & & 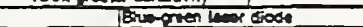 & & \\
\hline & \multicolumn{4}{|c|}{ 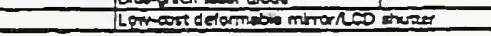 } & \\
\hline
\end{tabular}

\section{Rècommendations for Hardcopy Tethnology}

- Solid imaging

- Low-cost imaging system, including high-power UV laser and precision $x-y$ scanning; photopolymers for engineering plastics

\section{$\therefore$ Color standards}

- Research to determine a single, optimal color standard for all printing/scanning applications

\section{* Software}

- Development of software to enhance the ease of use of color, i.e automatic color rendering 


\section{LANCE Glasser, ARPA}




\title{
Administration Initiatives in Electronics
}
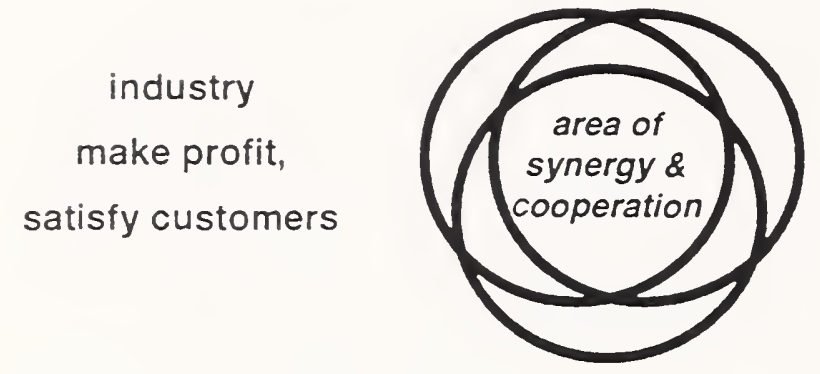

academia

educate students,

create knowledge

government

serve public good

\section{The Electronics Subcommittee}

\section{ESC}

\author{
Why the Federal Government is \\ concerned with Electronics
}

- Competitiveness in electronics manufacturing is critical to the Nation

- There are major competitiveness issues facing the U.S. Electronics industry

- Government must work with the private sector to facilitate long-term U.S. leadership and economic competitiveness in Electronics 
The Clinton Administration has established an Electronics Subcommittee (ESC) to help set priorities and coordinate Agency activities, comprising:

Office of Science and Technology Policy

National Economic Council

Department of Commerce

National Institute of Standards and Technology

Department of Defense

Department of Energy

Environmental Protection Agency

National Aeronautics and Space Administration

National Science Foundation

National Security Agency

Critical Technologies Institute of RAND-Non-voting participant

The right people; the right time; the right focus and Technology Council

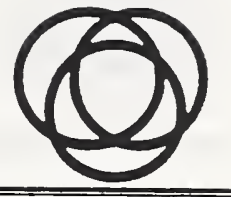


Provides interagency coordination for technical planning, budgeting, reporting, and evaluation of Federal programs in electronics science and technology and supports two-way communication on these programs within the government and with the private sector.

\section{ESC} ESC Functions

- Reports to the Civilian Industrial Technology Committee (CIT) of the National Science and Technology Council (NSTC) and represents the advocacy position for electronics issues with all Federal interagency crosscuts or initiatives.

- Provides coordination of interagency technical program planning, budgeting, and reporting, and coordination with the private sector, in order to encourage the development and execution of joint government-private sector electronics R\&D programs in priority areas.

- Provides a forum for government-private sector interaction

- Collects, maintains, and disseminates an up-to-date inventory of Federal electronics R\&D programs.

- Proposes and develops joint government/private sector initiatives in electronics. 


\section{ESC}

ESC activities and goals

\section{Forums for government-private sector} interaction

\section{Focused initiative development (NEMI)}

- Improve U.S. competitiveness and job creation in electronics and downstream industries

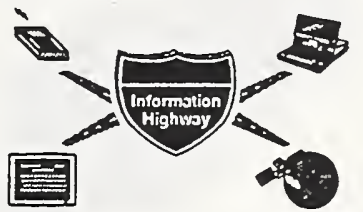

\section{Inventory project}

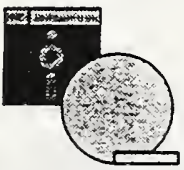

- Widely disseminate information on Federal government agency activities, priorities, strategic plans, and internal processes.

- Collect and maintain accessible data on electronics-related research and development activities in Federal agencies

\section{Benchmarking activities}

- Identify and measure key electronics competitiveness parameters

The right people; the right time; the right focus
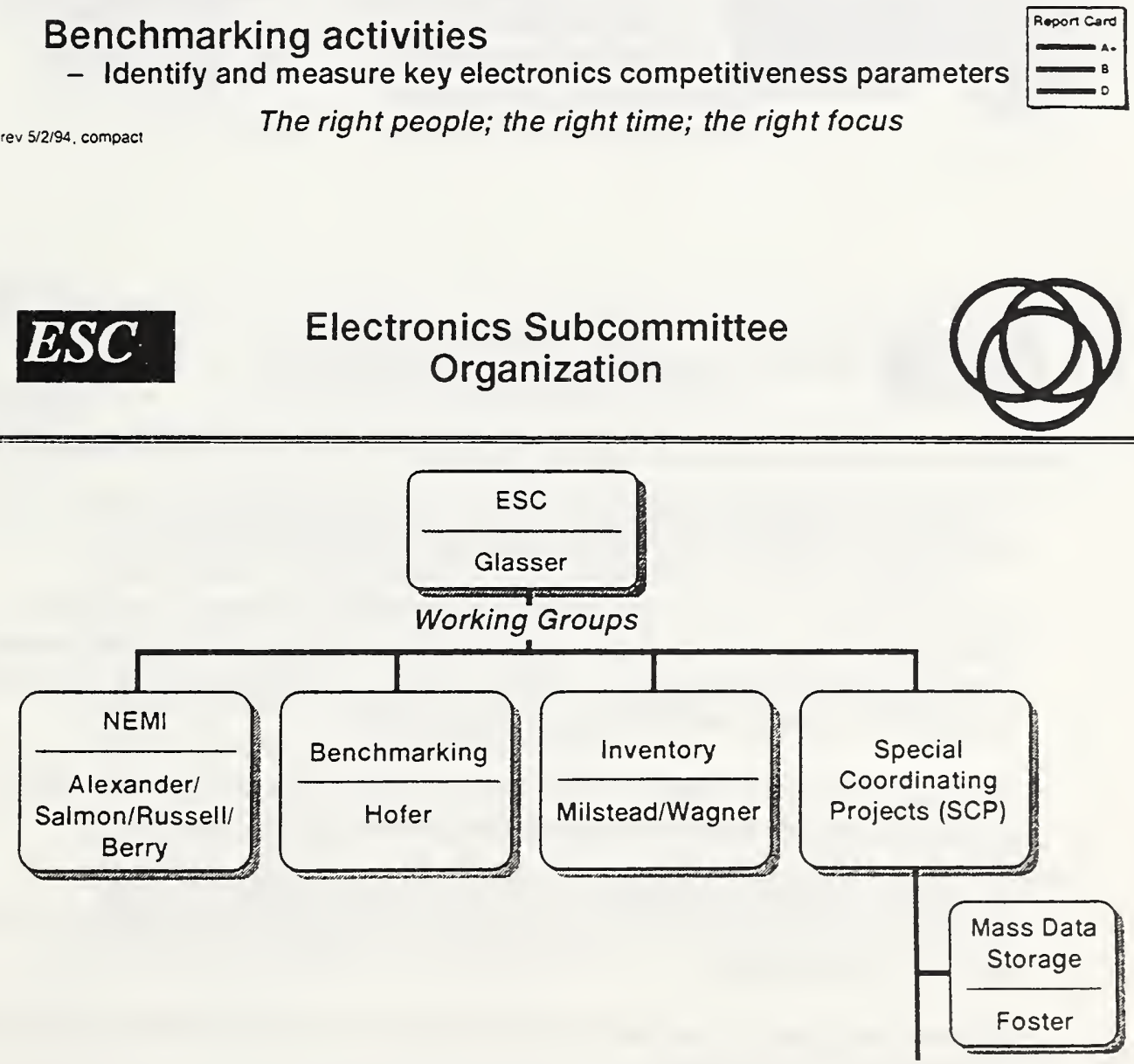


\section{ESC}

Tie four Major Government-wide Databases together. . .

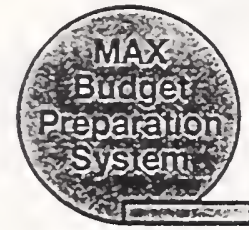

OMB

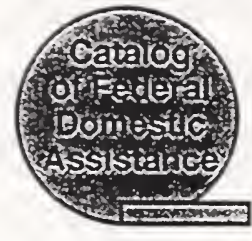

GSA

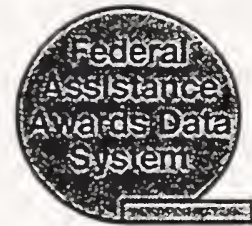

DOC

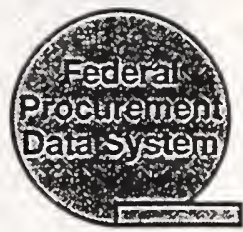

GSA

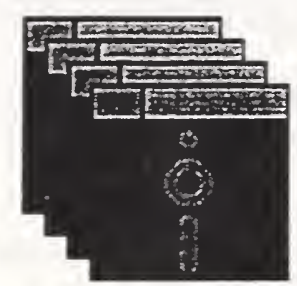

Augment with internal agency data collections:

DoD (R-1 w/ Descriptive Summaries, DROLS), HHS (CRISP), NASA (507 System), DOE (PADS), NSF (Mainframe), EPA (Awards System), DOD (Internal Files), USDA (CRIS), etc.

The right people; the right time; the right focus

ESC

The concept of an abstract product focus

- The electronics industry is too broad and diverse to be encompassed by a single strategic plan or vision

- By picking a class of products on which to focus, one can develop a workable plan for infrastructure, policy, and research that supports the product class, with priorities and action items for government and the private sector

- The government does not actually build the products, which are only an abstraction used for planning purposes

- Industry builds products, with their own funds, on the infrastructure developed and within the policy envelope of the planned initiative

- Moreover, the initiative is expected to have benefit beyond the selected focus area 


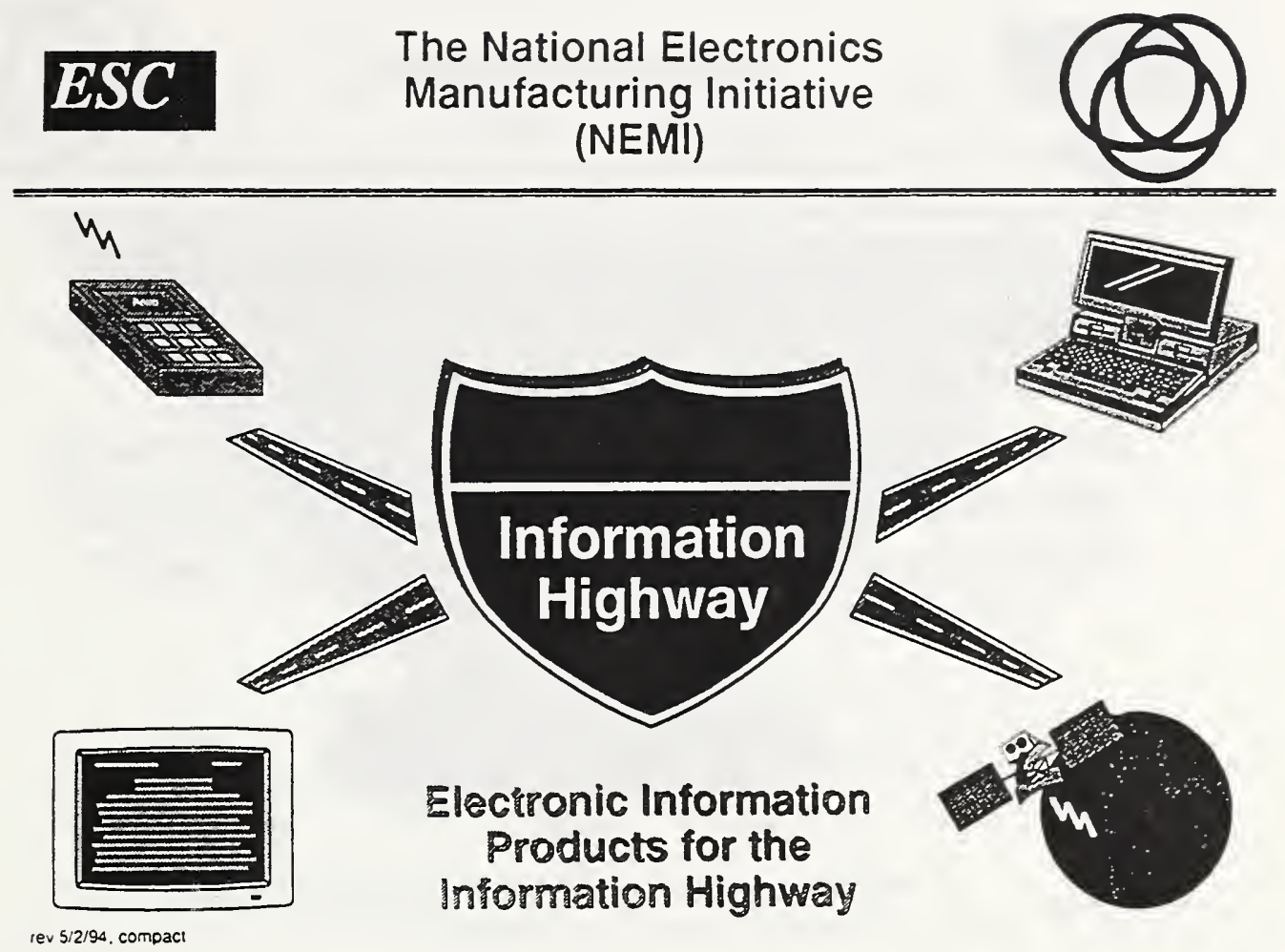

ESC

We have a vision for a focused Electronics Manufacturing Initiative

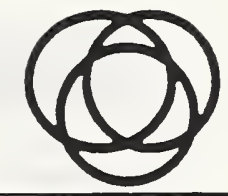

- Focus on electronic information products for the information highway - the National Electronics Manufacturing Initiative (NEMI).

- Government would provide support for enabling technology and infrastructure.

- Can deploy some existing government resources in FY94/95 (such as ATP or TRP) if the industry becomes enthusiastic and if a good plan is developed. On track to impact FY96 budget cycle. 
Led by Vice President Gore, the United States is embarking on an aggressive program to network the nation with 21st-century information "superhighways." What hardware and software will connect to these information highways and which nation will make them?

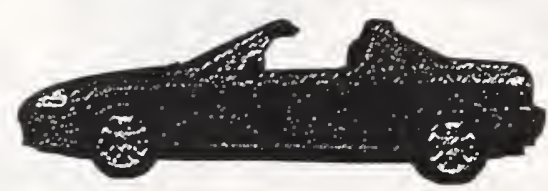

The right people; the right time; the right focus

\section{ESC}

Attributes of Electronic Information Products that will drive technology decisions over the next decade

Delivers multiple information services to people 䎠圄 User interface centered

Connected to international-scale information infrastructures

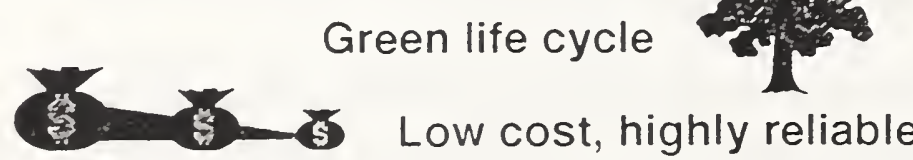

Ubiquitous, huge but differentiated markets

Often mobile, portable, lightweight
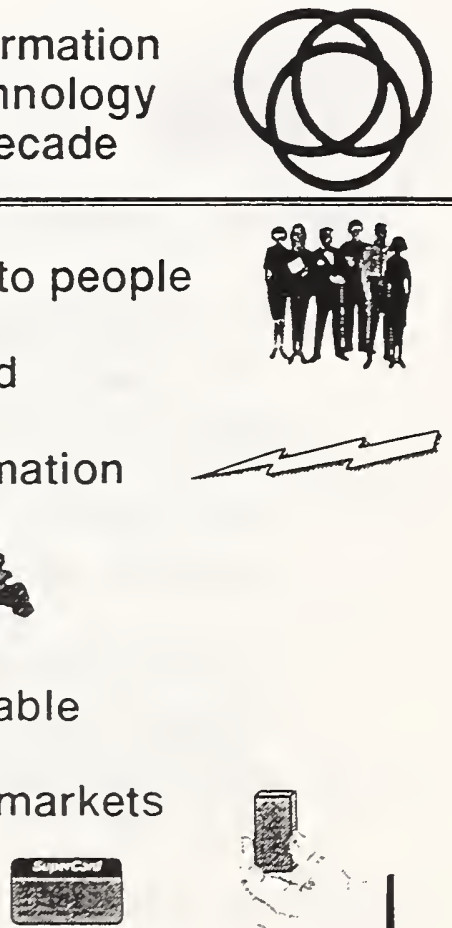


\section{ESC}

Focusing on make-a-difference

$R \& D$ investments

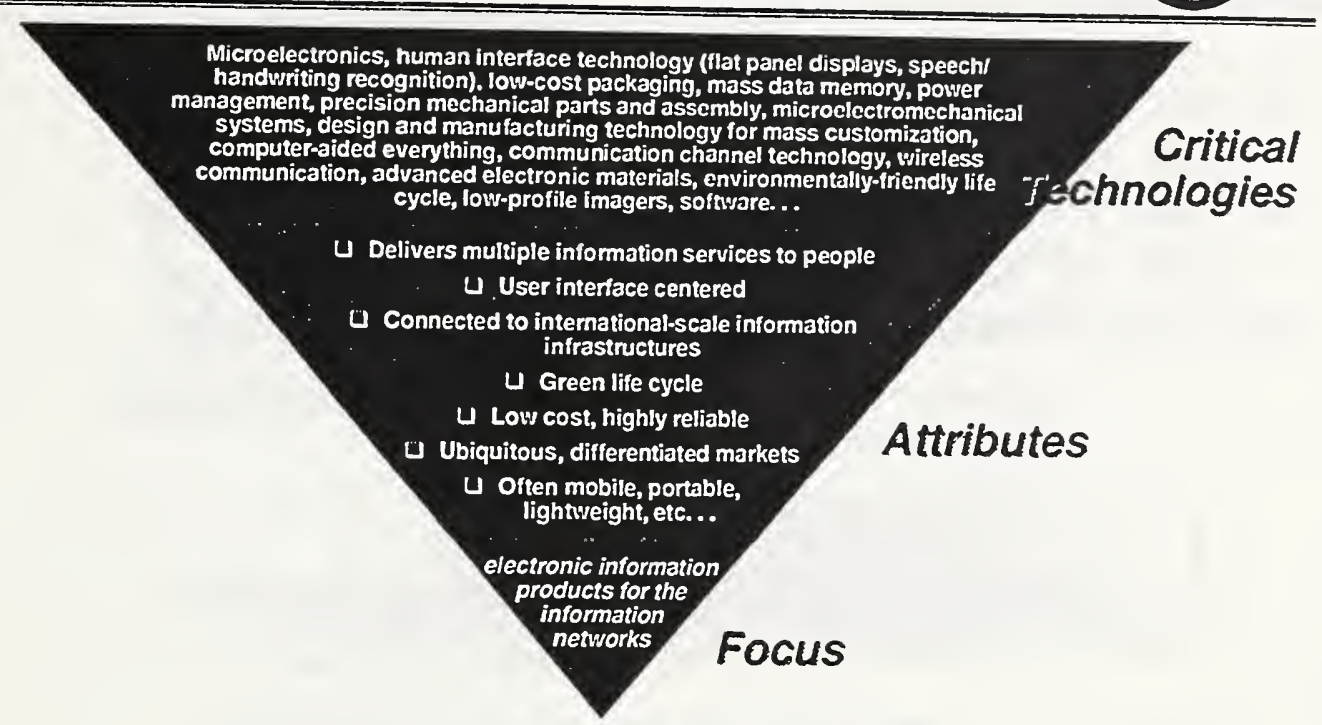

The right people; the right time; the right focus

Policy issues will be overseen by the

ESC National Economic Council and the Office of Science \& Technology Policy,

e.g.,

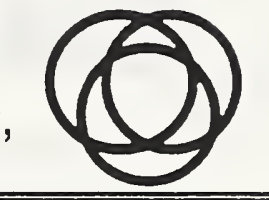

- USG procurement

- Environmental regulation

- International technology transfer

- Technology-for-technology initiative

- U.S.-Japan technology framework negotiations

- Federal-States cooperative efforts

- Trade

- Export promotion

- Export control

- Facilitating Standards

- Labor issues specific to high-tech manufacturing

- SBIR increase and its relationship to Administration initiatives

- Intellectual property issues

- Infrastructure, such as NII or manufacturing extension services 
Examples where the Federal government could be an early customer:

- Very low cost electronic tutors/assistants for accelerating education and training

- Distributed environmental sensor networks

- Systems of sensors and actuators for Federal building energy savings

- Smart National health cards

- Hand-held Military Information Associates

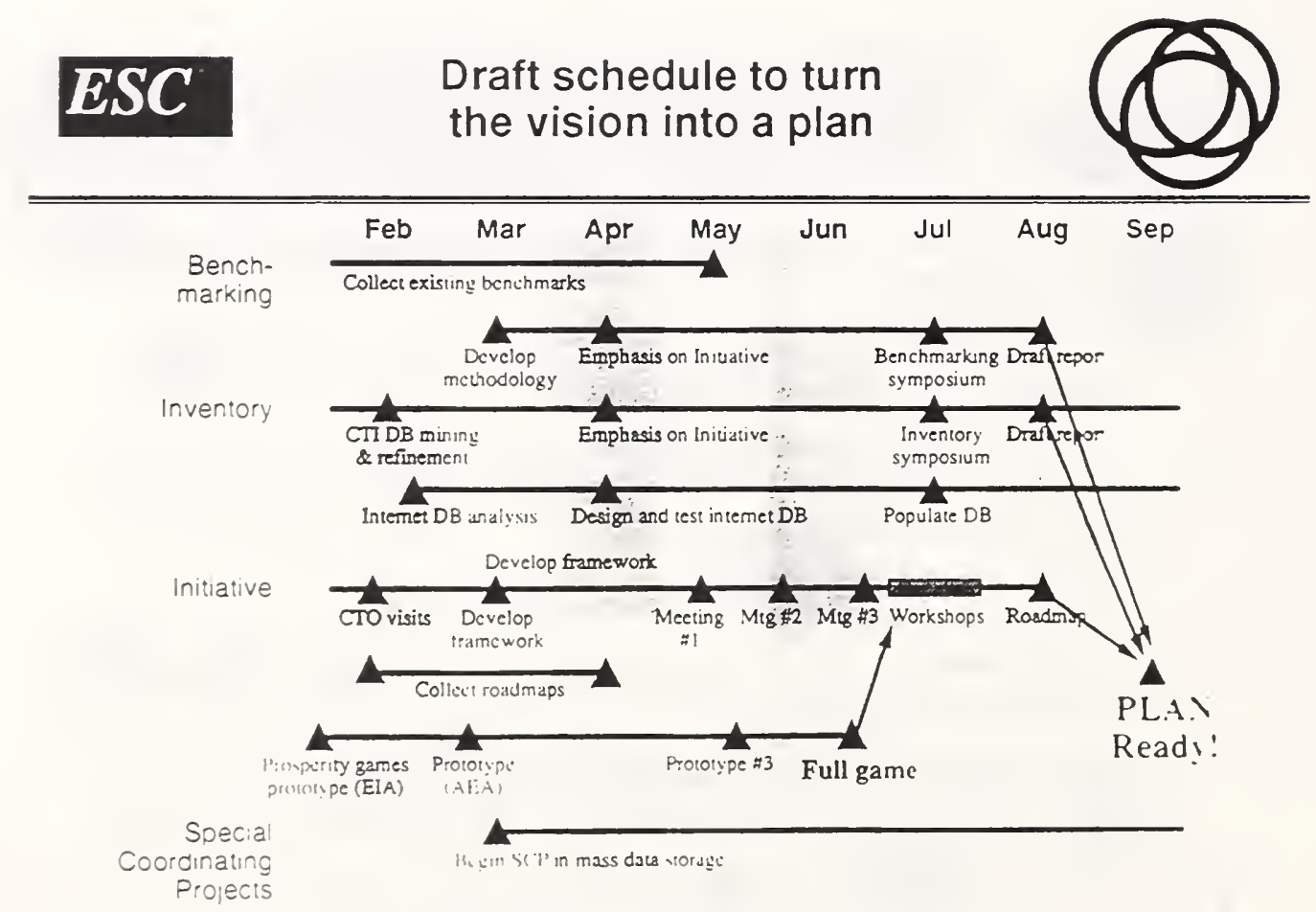


When any business, anywhere in the world, confronts a decision on where to locate, be it for research, design, manufacturing, or service, the clear choice is the USA because we have both the best environment for business and the best environment for life.

\section{This will result thildobs, \\ Competitiveness, and Public Good:}

The right people; the right time; the right focus

ESC

The ESC needs feedback

Electronic Intormation

Venicles for the

information Highway-

A new electronics

manufacturing

initiatuve 

NEMI Framework Process

Leading large and small companies intimately connected with the electronics industry:

Apple Computer Inc.

AT\&T

Compaq Computer Inc.

Critical Technology Institute/RAND

Digital Equipment Corporation

Intel Corporation

IBM

Microsoft Corporation

Motorola
National Semiconductor

Philips Corporation

Silicon Graphics

Texas Instruments

Westinghouse

Xerox 
- Competitiveness in electronics manufacturing is critical to the Nation

- Largest U.S. manufacturing sector with 2.4 million American workers

- Upstream of many key industries, e.g., computer, telecom, auto

- Essential to National security

- There are major competitiveness issues facing the U.S. Electronics industry

- Consistent bilateral trade deficit with Japan of $\$ 20 B$

- $20 \%$ of total U.S. global trade deficit

- U.S. global market and manufacturing shares dropping

- New business challenges loom, e.g., green life cycle constraints, exploding capital equipment costs, shrinking product lifetimes, increased corporate networking

- New technology challenges loom, e.g., new technology drivers

- Government must work with the private sector to facilitate long-term U.S. leadership and economic competitiveness in Electronics

- The government has a role, whether it means to or not, through infrastructure, regulation, tax and trade policy, R\&D investment, etc.

- The ESC believes that the government can have a constructive role on competitiveness by

" working in partnership with the private sector

" investing in R\&D and infrastructure

" addressing policy issues

The right people; the right time; the right focus

- Electronics Industry was the largest industrial employer with 2,400,000 jobs in 1991

- It is the largest and the fastest growing U.S. manufacturing industry, projected to reach $25 \%$ of all U.S. manufacturing in 1995

- Electronics is key to competitiveness in other sectors such as Computer, Consumer, Telecom, Automotive, Aerospace, Chemical Process, and Medical

World electronic products and services market

$$
\$ 2,000-
$$

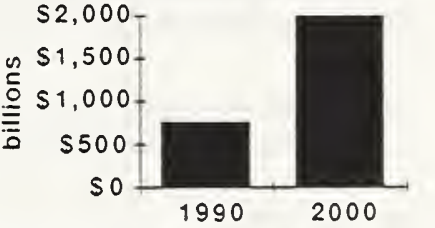

Automotive electronic content

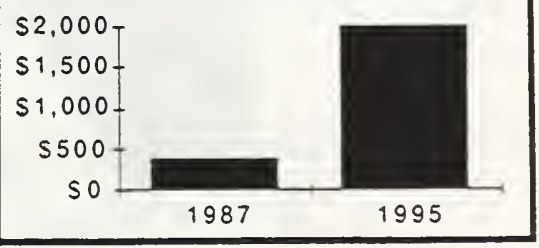

SOurces SEMATECH, NACS EIA DOC AEA. Lallas Moming ivens 

of pervasive impact

Some of the Industries that Electronics impacts:

- Computer

- Telecommunications

- Education

- Automotive

- Entertainment

- Industrial Control

(e.g., for Food and Chemicals, Buildings)
- Aerospace

- Consumer

- Medical

- Defense

- Environment

An electronics initiative should matter to and be engaged with a number of these customers. It must make a difference beyond just the electronics industry.

An Administration initiative should also be larger than what a single agency or the private sector can accomplish alone.

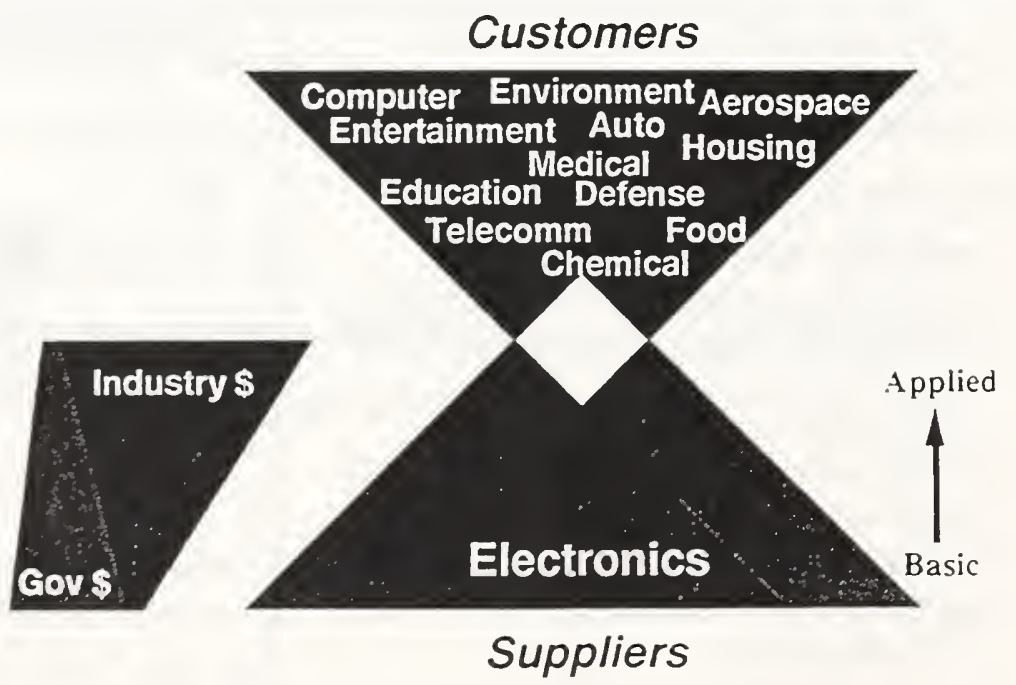


- There will be a revolutionary increase in information connectivity. For example, the wiring of the nation with cable and optical fiber and the work on HDTV are harbingers of these changes.

- There will be a revolutionary increase in mobile information services. For example, the explosion in cellular telephone usage and FCC actions on PCS (Personal Communication Systems) spectrum foreshadow even greater changes to come.

- There will be a revolution in innovation. Information devices are being redefined and resculpted in new and creative forms. Scanners are merging with photocopiers, cellular fax modems are being incorporated in portable computers, advanced automobiles are $30 \%$ electronics, TV is merging with personal computers, and new devices, such as the personal digital assistant, are coming on the market.

- There will be increasing emphasis on green manufacturing and life-cycle environmental considerations, including cradle-to-grave product management.

- Components are getting smaller and more sophisticated while demands for quality and low cost are increasing, making automated assembly more desirable than low-cost manual assembly.

rev 5/2/94, compact

ESC

Discontinuities, continued

- Customers are demanding more integrated products while companies are doing more outsourcing and networking. With more interfaces among companies, government effects on the business environment are even more important.

- Consumer electronics products are becoming technology drivers, often containing, and being manufactured with, the most sophisticated technologies.

- Developing countries will leap into new and modern infrastructures that will use advanced devices.

- Product development times and lifetimes continue to decrease as capital equipment costs explode. Risks increase.

\section{Discontinuities provide the opportunity for make-a-difference investments}


- Focus on electronic information products for the information highway

- Will be a market and technology driver for electronlcs manufacturing over the next decade

- Support Clinton Administration priorities, not only the National Information Infrastructure (NII), but also, potentially, the clean car, health care, and education.

- Encompasses many of the technologies critlcal to success in all areas of electronics, e.g., packaging, semlconductors, dlsplays, preclslon assembly, manufacturing, environmentallyconscious electronics manufacturlng techniques; design for cradle-to-grave, batteries, robotics

- Government would provide support for enabling technology and infrastructure

- Basic technology R\&D. High risk investments for fundamental change.

- Investments whose benefits private investors cannot hope to appropriate, but that a nation can.

- Investments that serve a public need.

- Infrastructure investment, e.g., Metrology. Machine tools and Equipment. Computer aids.

- Technology deployment and extension services. Benchmarking.

- Workforce education and training.

- Mission-specific prototyping and procurement. (Defense, Education, Environment, etc.)

- Can deploy some existing government resources in FY94/95 (such as ATP or TRP) if the industry becomes enthusiastic and if a good plan is developed. On track to impact FY96 budget cycle.

- The basic product and process technologies

- Ranging from the fundamental to the applied

- For creating future world-class Electronic Information Products for the Information Highway

- Within a customer context supplied by nationally important industry clusters

- Consistent with key public goods such as education, health, environmental consciousness, and national security

- With government investments that can make a difference over the long term

- In partnership with the private sector 
MARSHALL ANDREWS, ITRI 


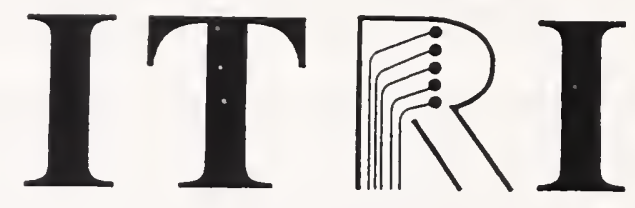

INTERCONNECTION

\section{TECHNOLOGY \\ RESEARCH \\ INSTITUTE}

\section{IPC}

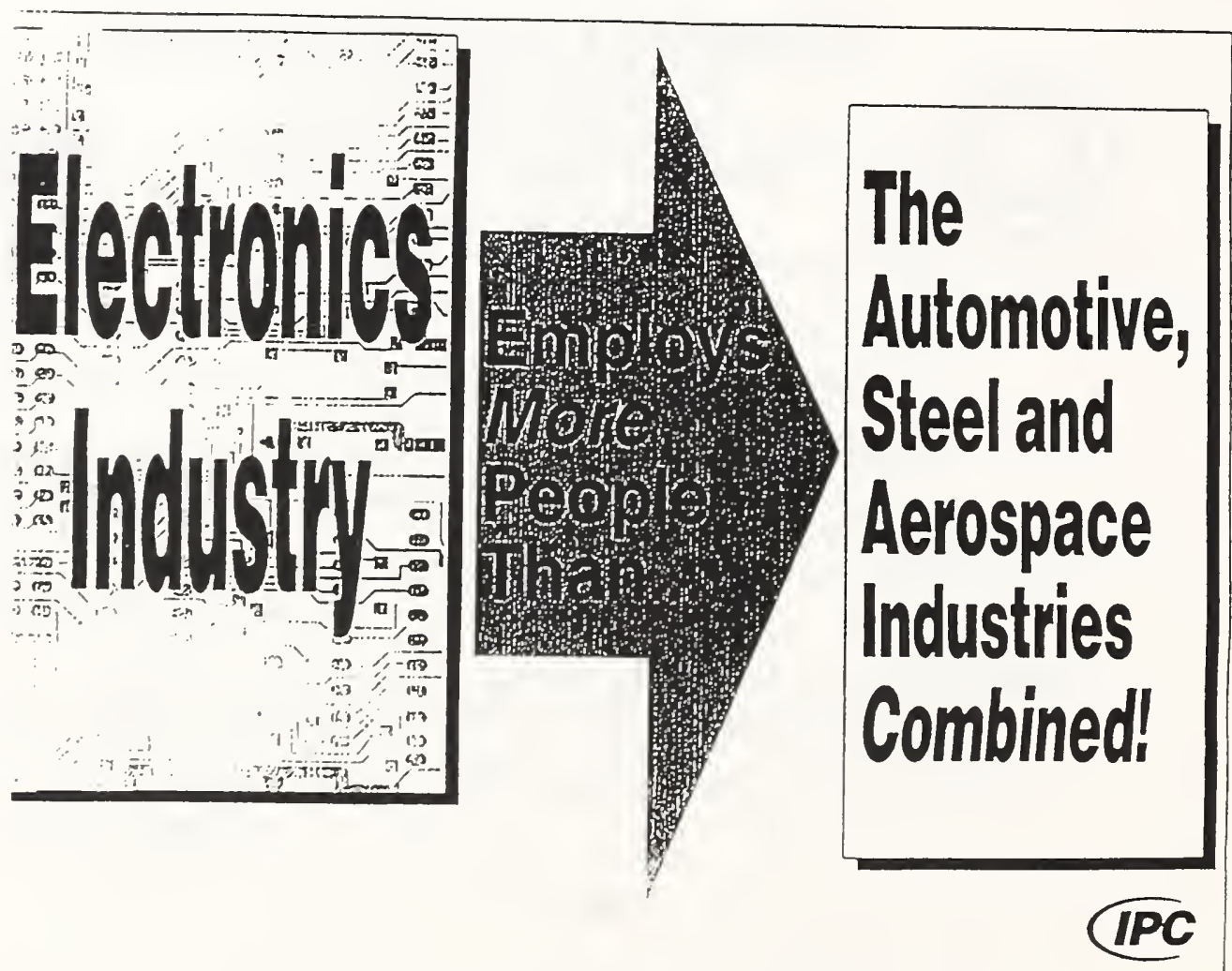




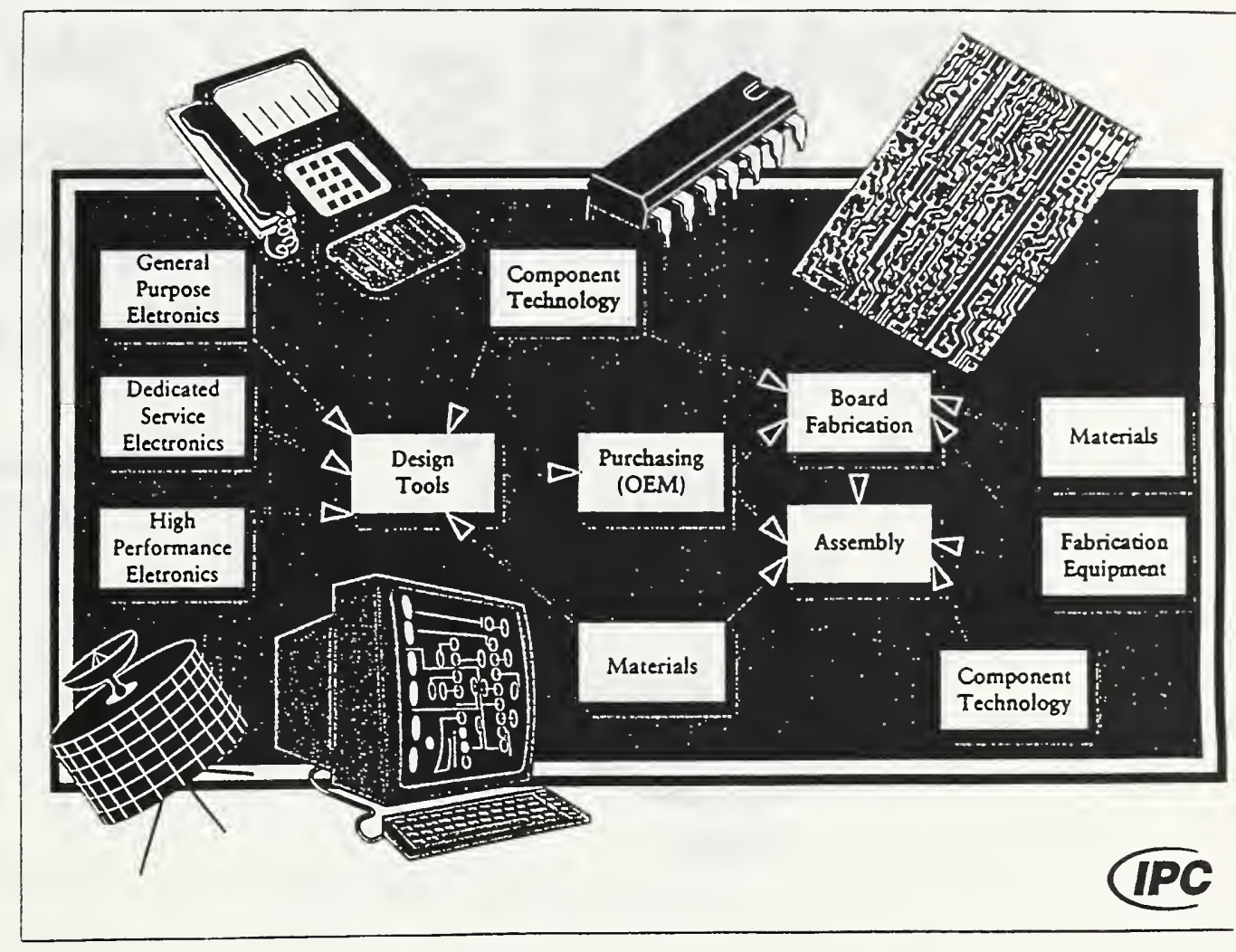

\section{U.S. PWB and Assembly Industry}

\section{PWB Manufacturing}

- 5.9 Billion in Sales Annually

- Over 100,000 Jobs, Including Those of Industry Suppliers

\section{Including PWB Assembly}

- 12 Billion in Sales Annually

- 200.000 Jobs in U.S. 

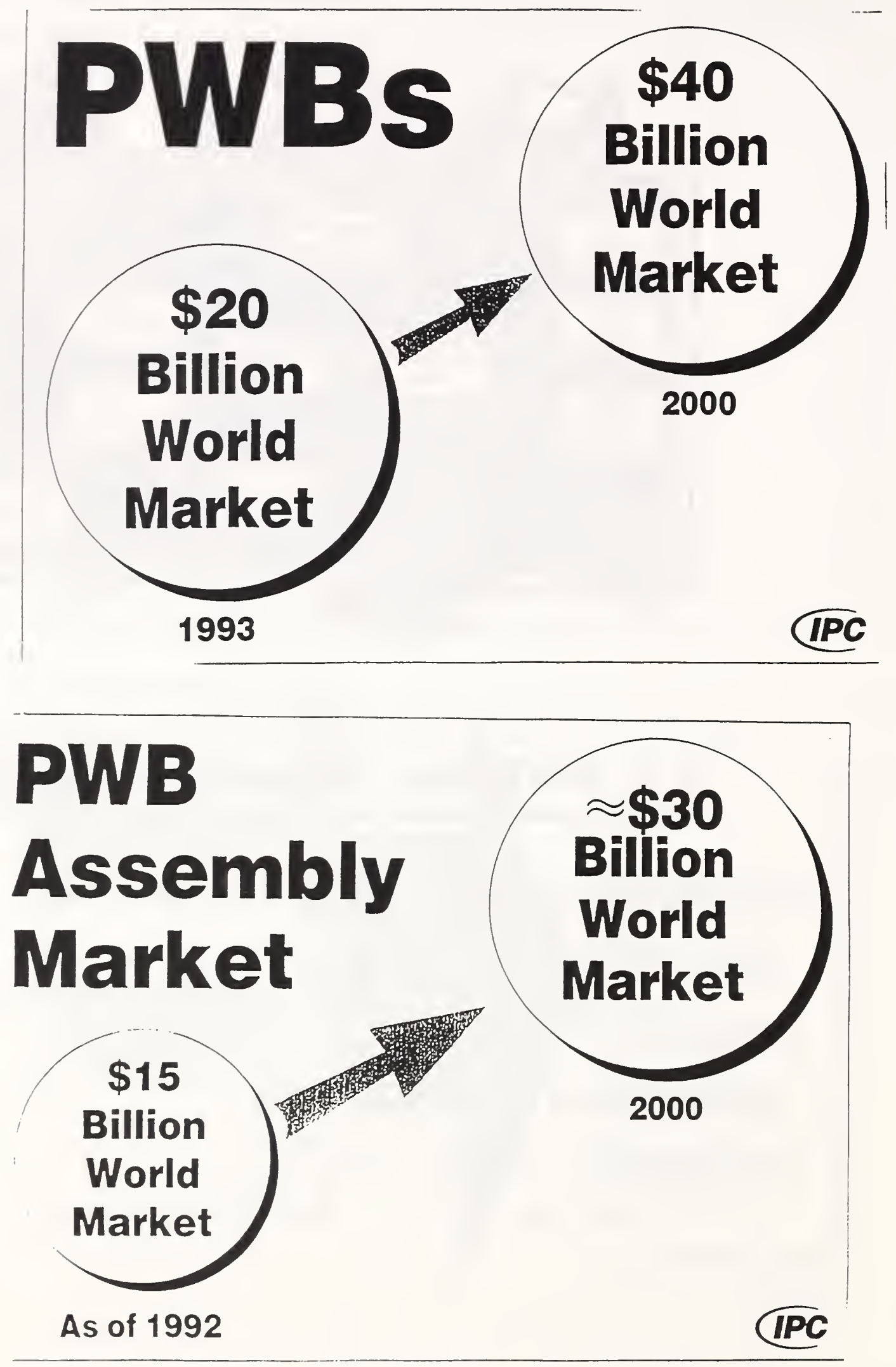

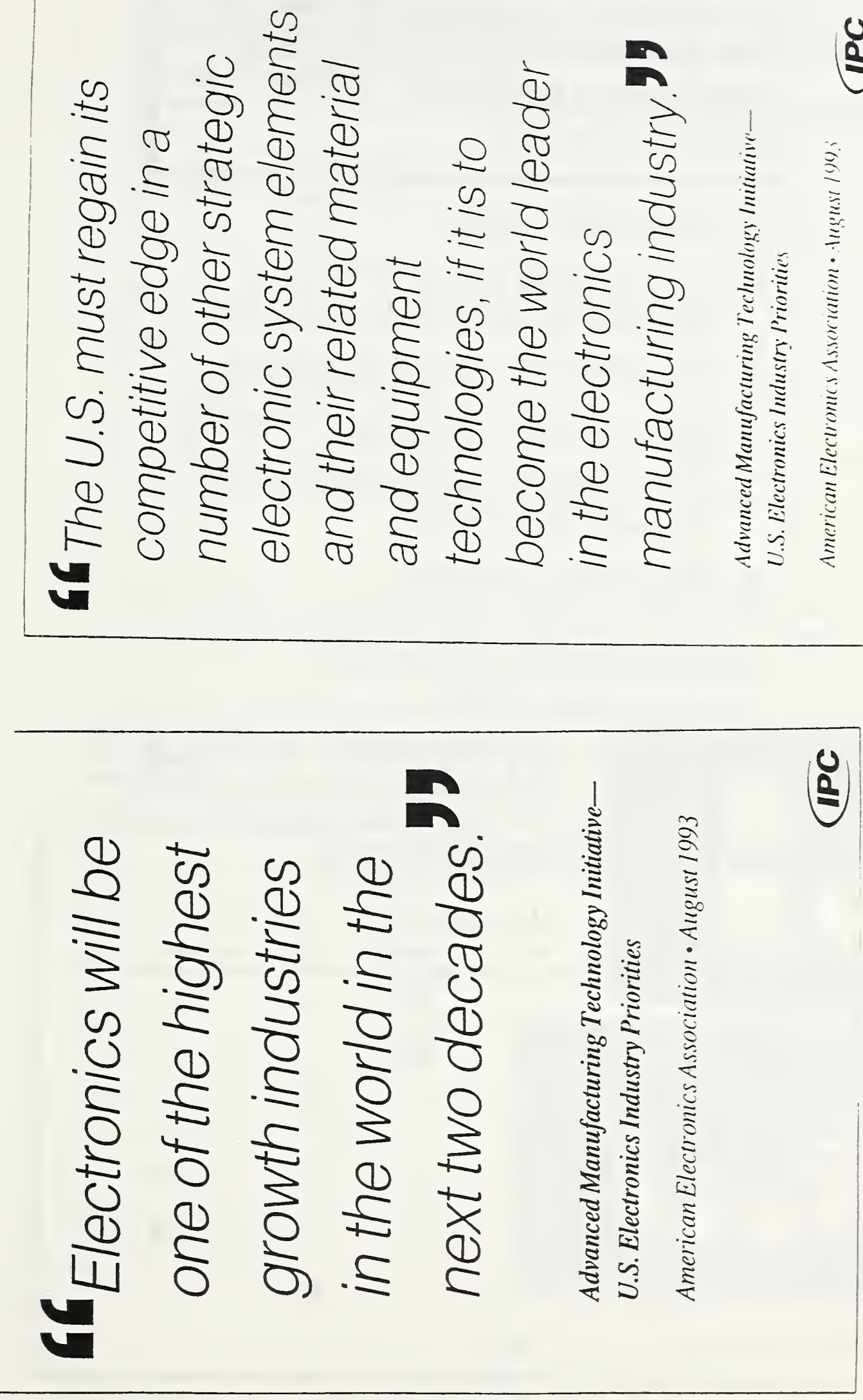
Six electronic system elements are widely recognized for having the greatest impact on the U.S. electronics industry. (In order of priority:)

1. Integrated Circuits

2. Printed Wiring Boards (PWB) and Multichip Module (MCM) Substrates

3. Liquid Crystal, Flat Panel Displays

4. Semiconductor Packaging Technology

5. Printed Wiring and Substrate (MCM) Assembly Technology

6. High Density Batteries

Advanced Manufacturing Technology Initiative-

U.S. Electronics Industry Priorities

American Electronics Association • August 1993

IIPC

\section{Electronic Components}

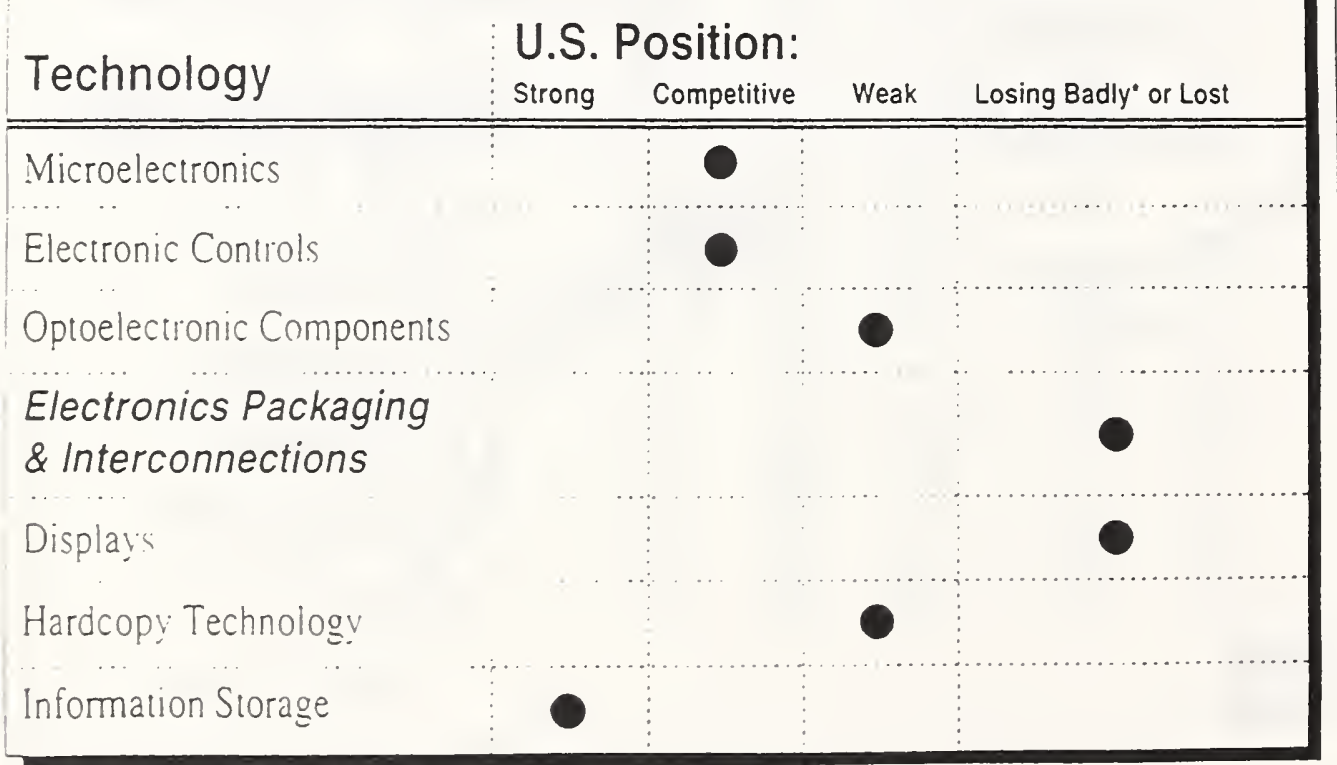

*U.S. Industry is no longer a factor or is not likely to have a presence in the next five years. It will take considerable effort or a major change in technology for the U.S. 10 become competitive. 


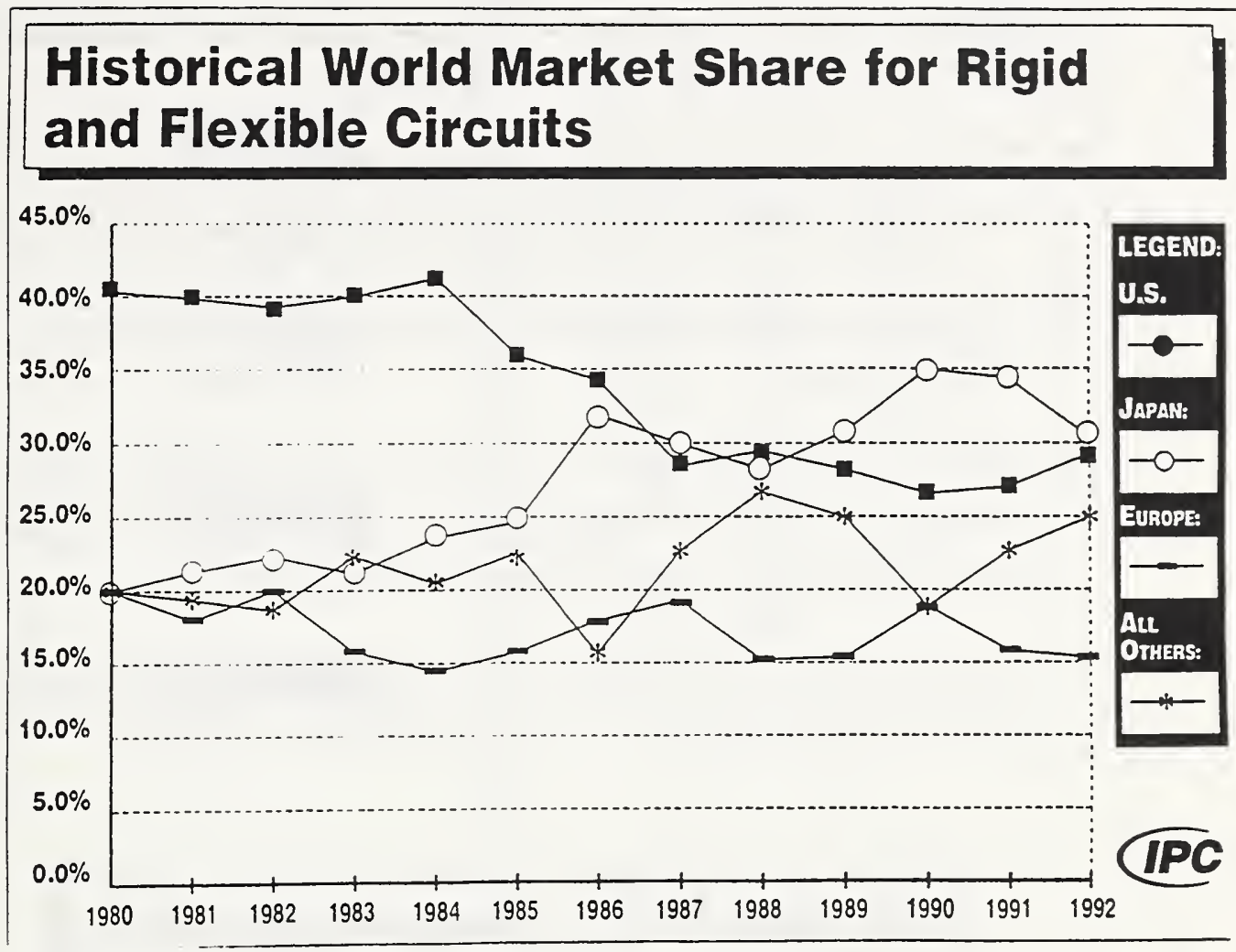

\section{U.S. PWB Industry}

Trends in Industry Structure

$90 \%$ of Independen PW B Manuficlurers

have ammal vilco ol $<\$ 10$ Million.

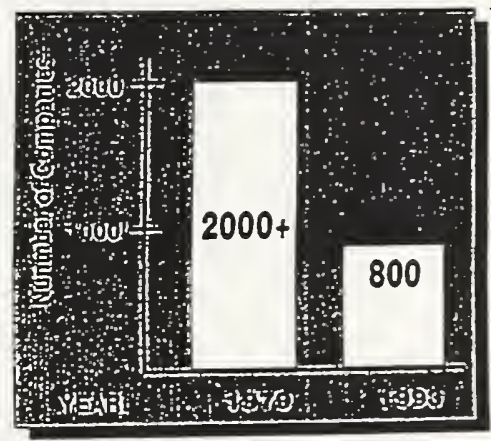

Low milngins mathe ciapilal investments difficult. Expenditure on $R$ \& D assumed to be decreasing with the trend toward outsourcing by OEMS.

Who will do hlu R \& D mecessill! 10 regain compethituchess of this critical $1: .5$. Industry?

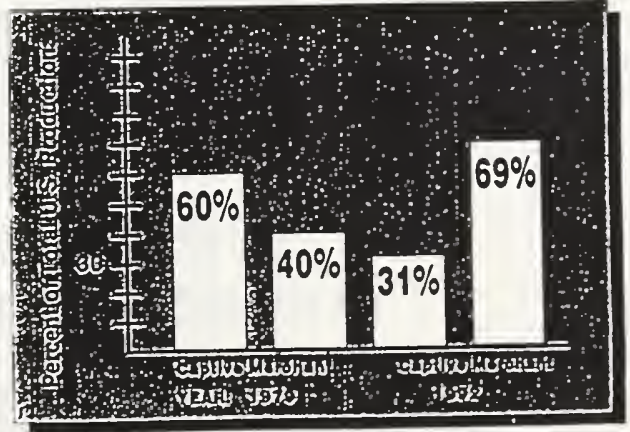




\section{Printed Wiring Boards \& Printed Wiring Assembly}

Critical Technology for the United States

- PWBs and PWBAs are pervasive in electronicsnot much you can do electronically without the PWB.

- Provide the interconnection between advanced ICs and humans.

- Multi-use technology is a major component of most electronic systems: computers, communications, automotive, military, industrial and consumer.

- Coming up on the screen as a major showstopper and critical technology for U.S. electronics industry.

\section{Competitive Requirements}

- PWB and PWBA Research and Development to find less costly ways to make interconnection products

- U.S. Leadership in Supporting Infrastructure Industries and Technologies

- PWB Fabrication Materials

- PWBA Assembly Materials

- MCM Substrate Materials

- PWBA Assembly Equipment

- PWB Fabrication Equipment

- PWB Test and Inspection Equipmeni

- Volume Manufacturing Capability

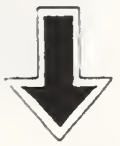

\section{Competitive U.S. PWB and PWBA Industry}




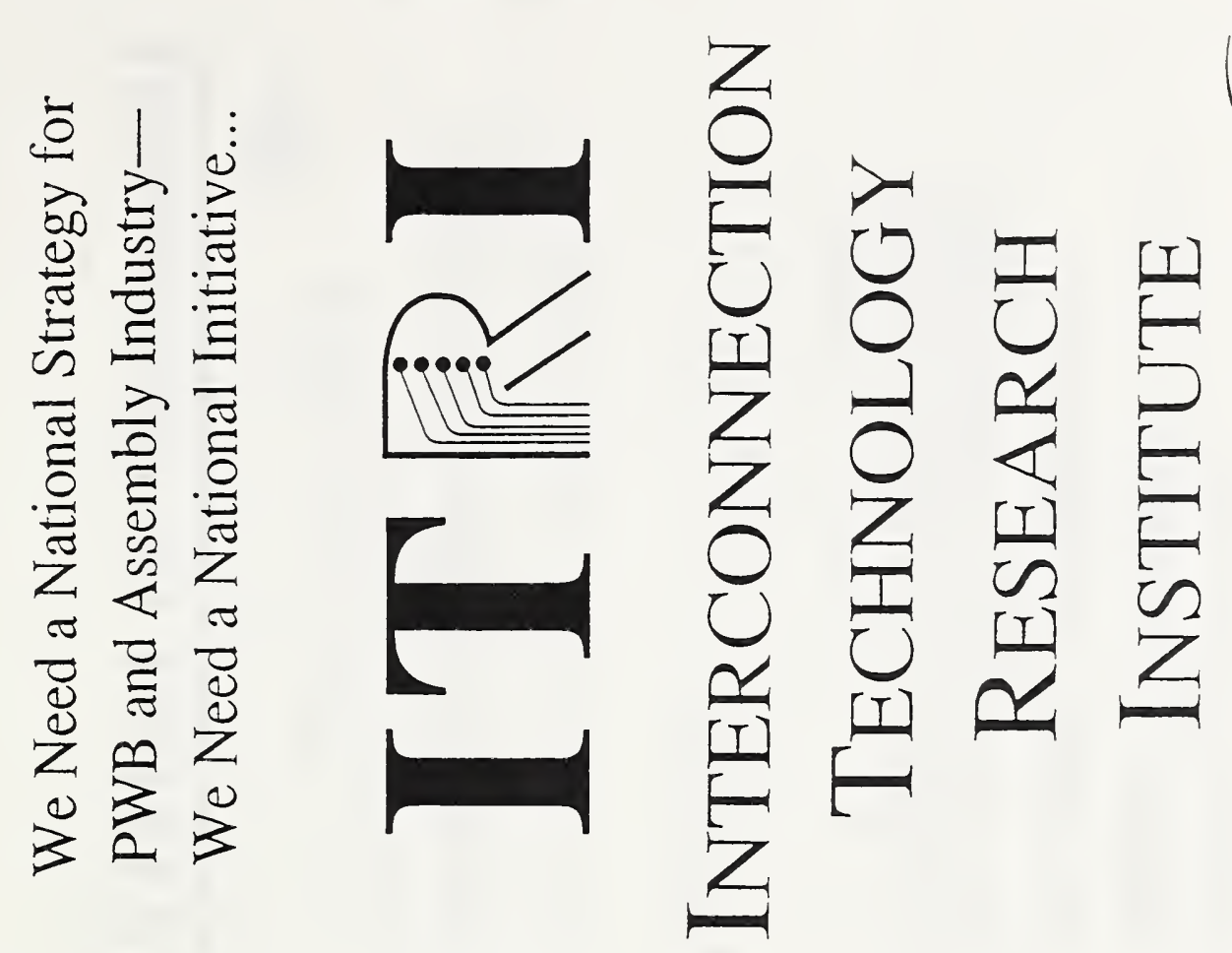

0

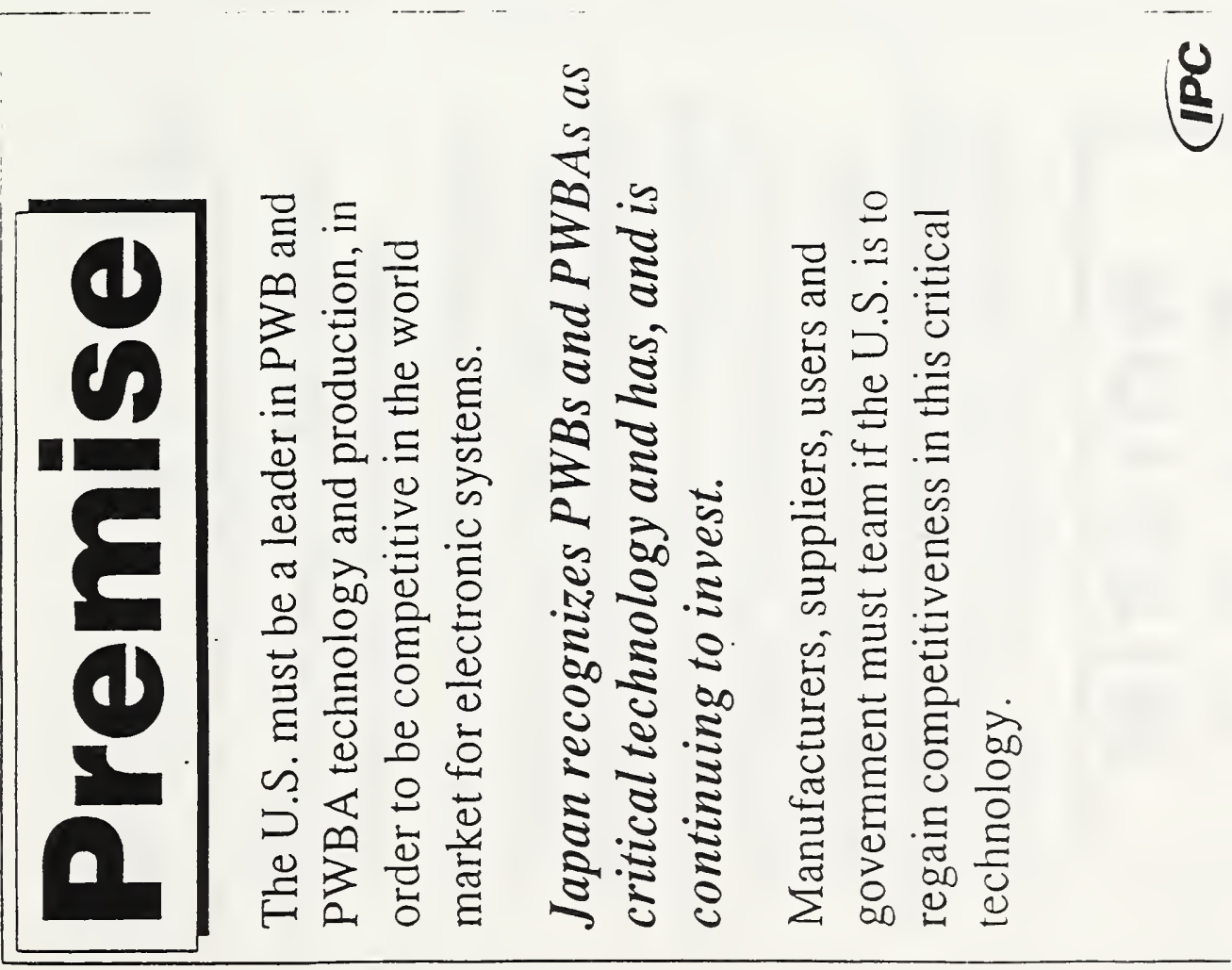



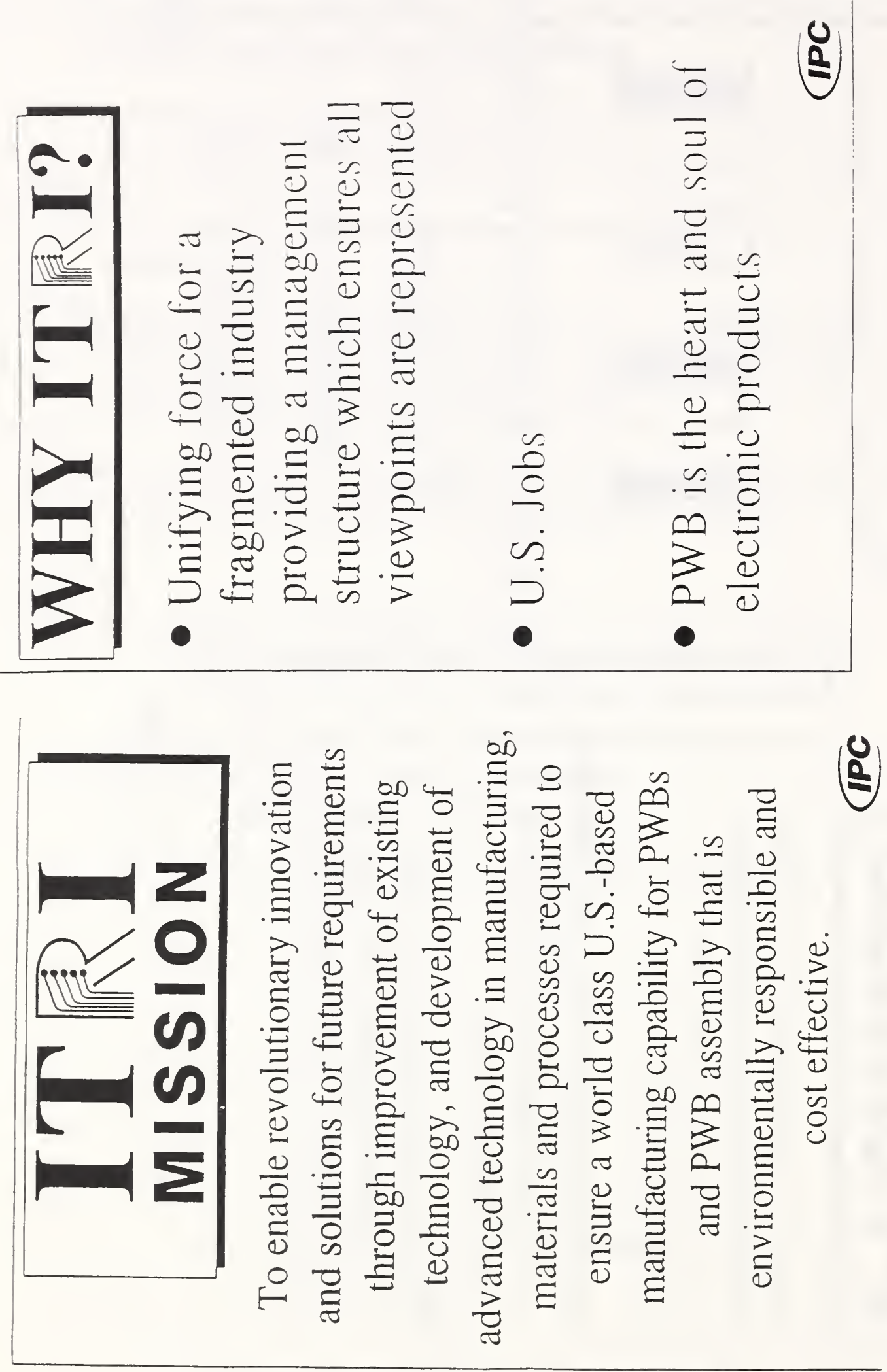


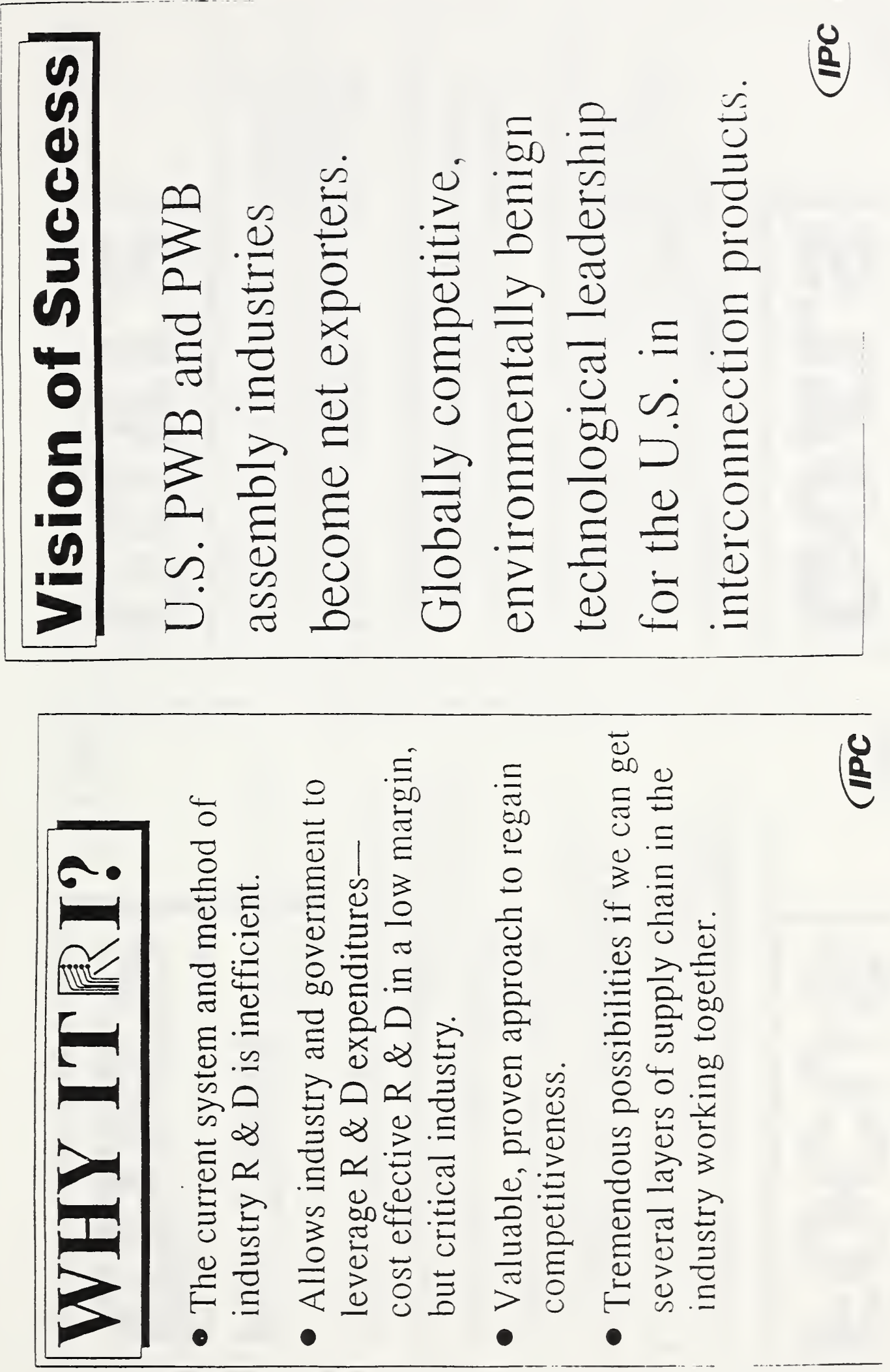



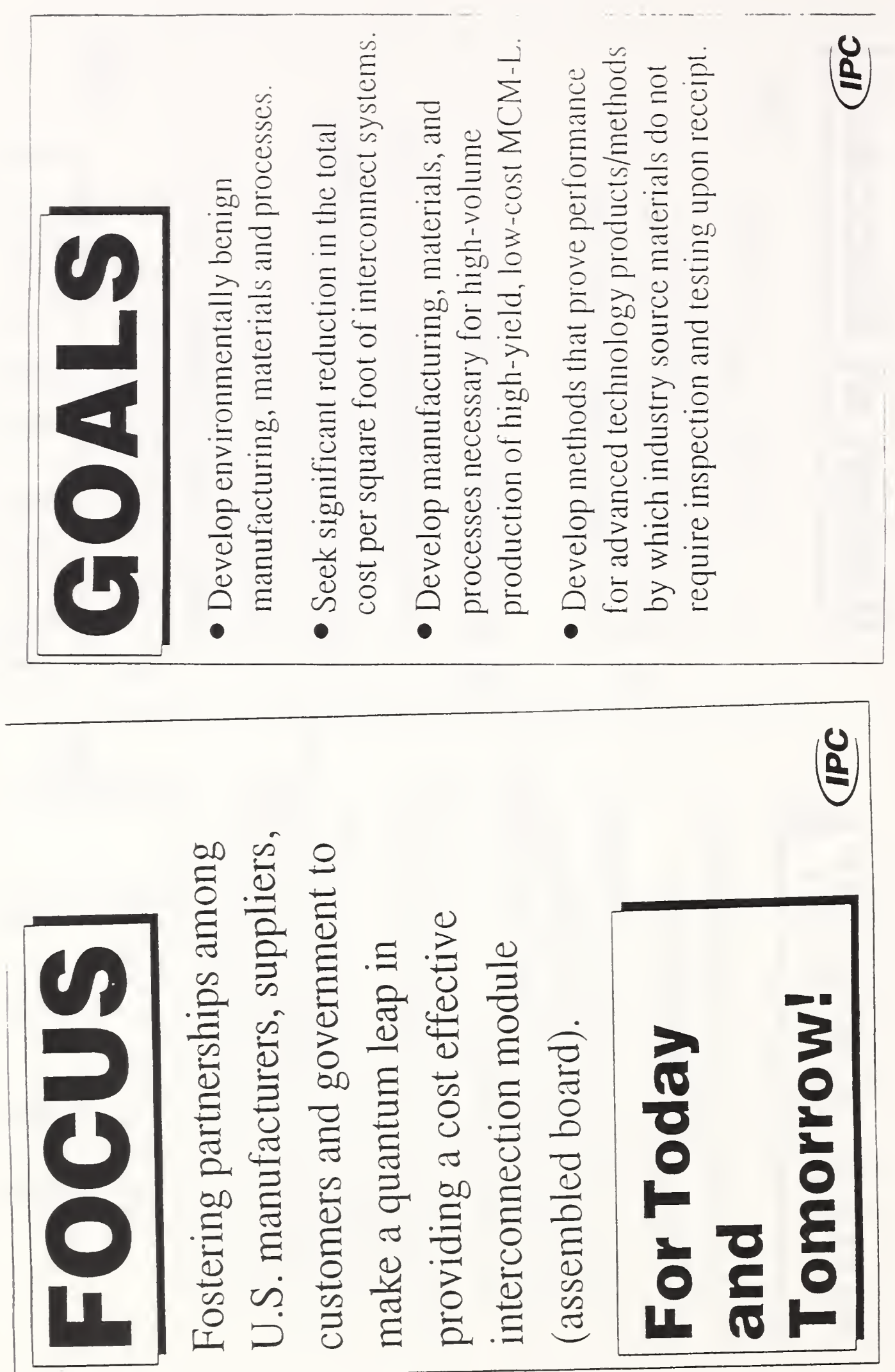


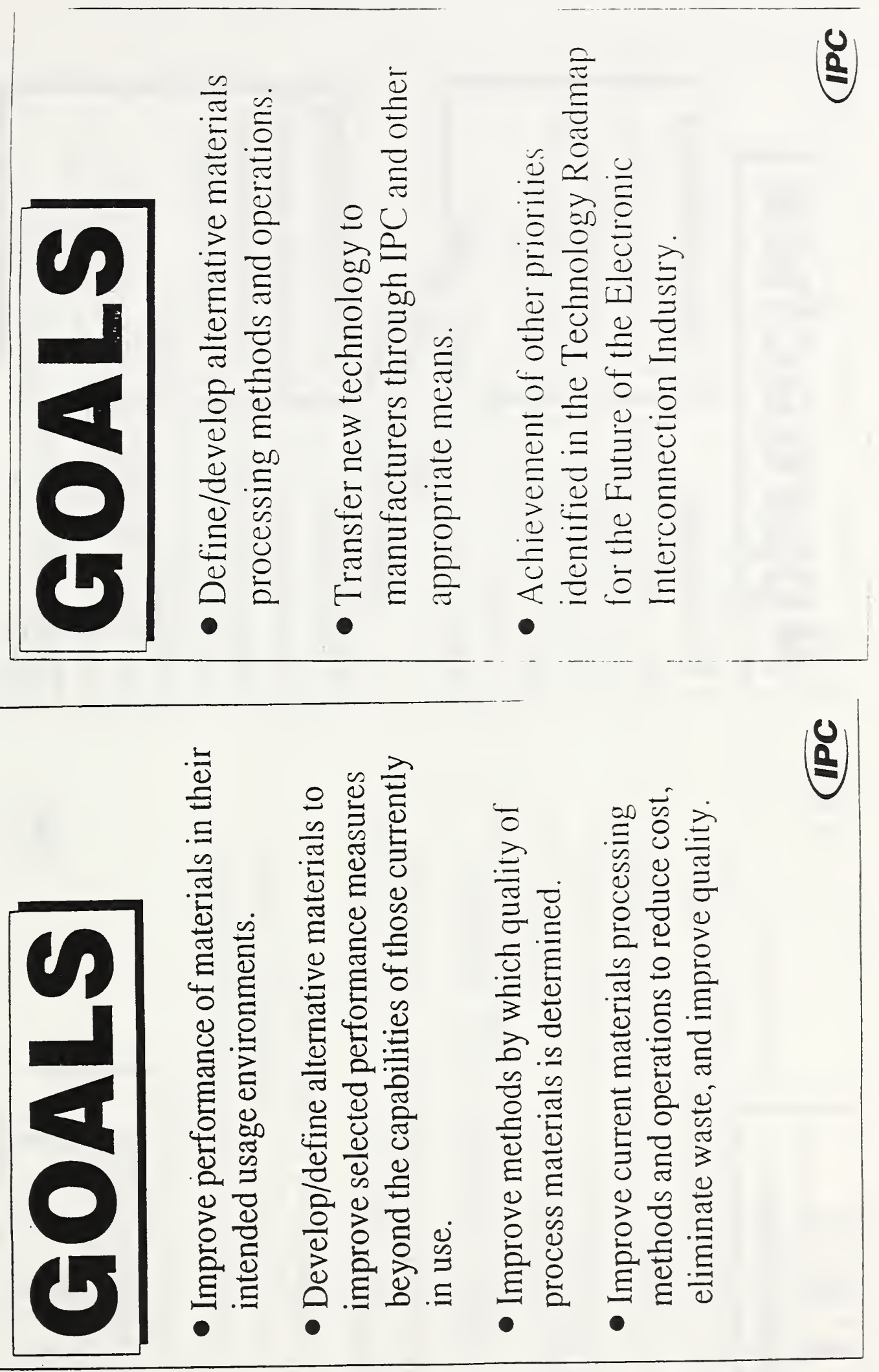



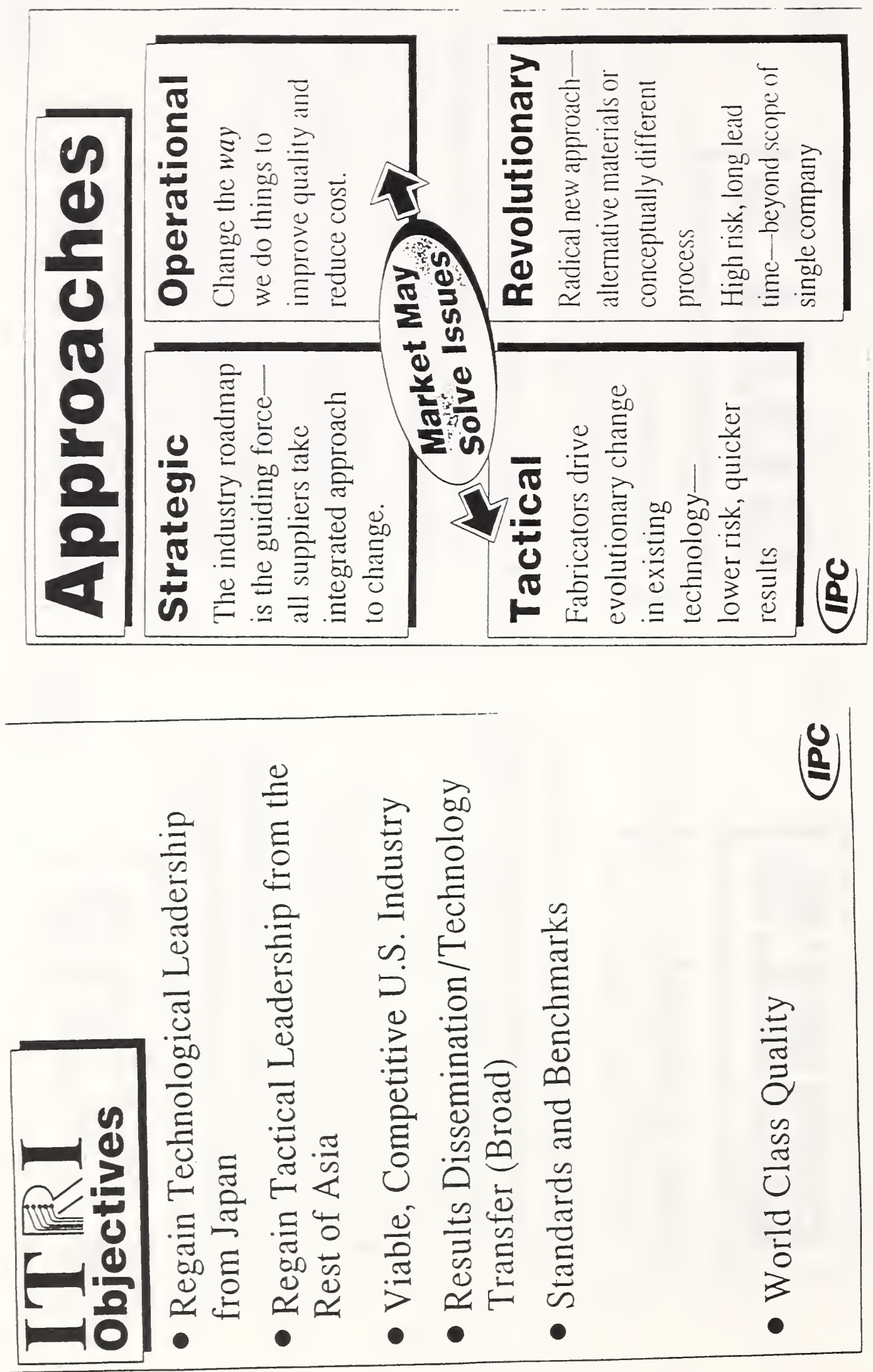

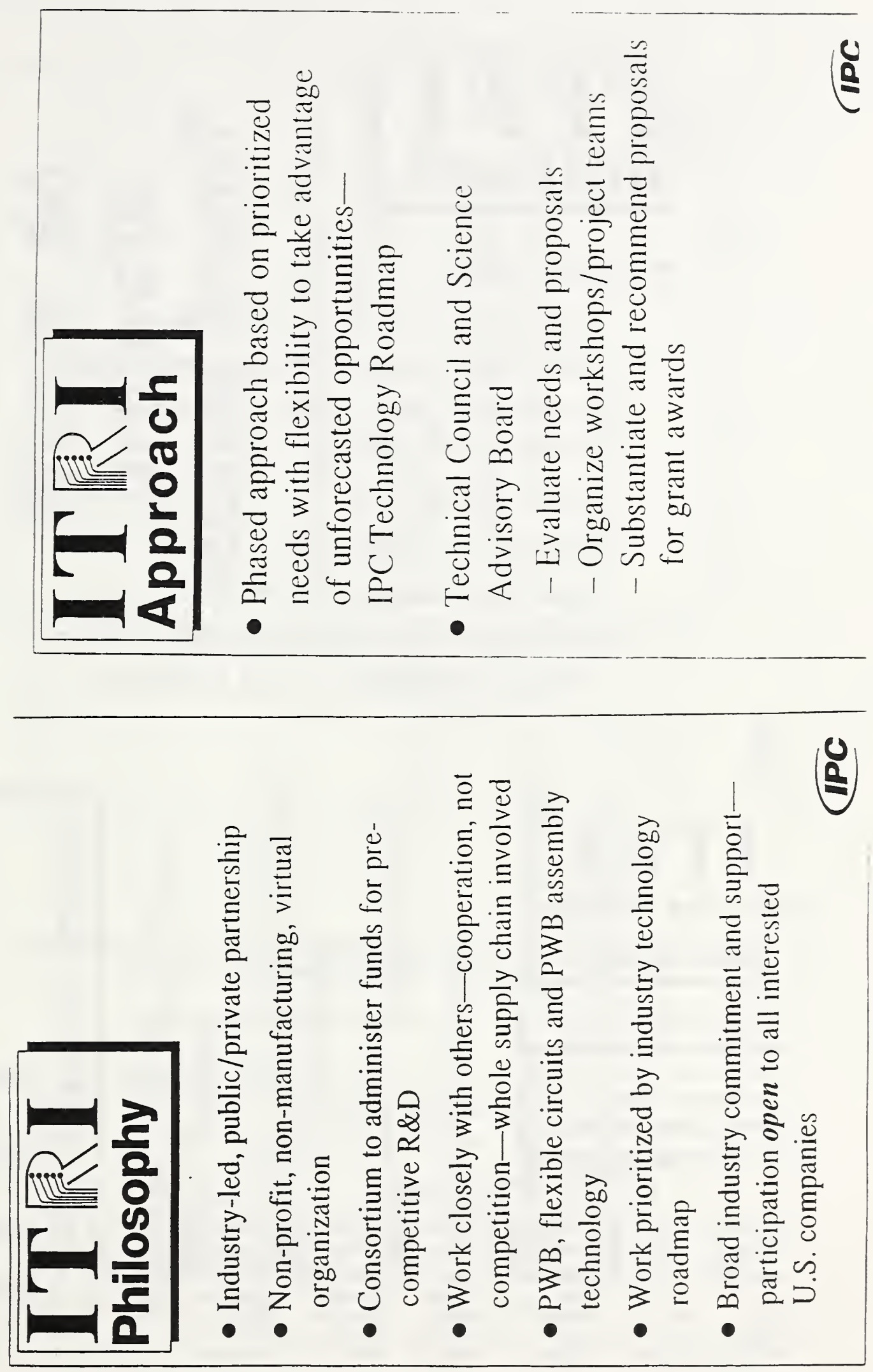


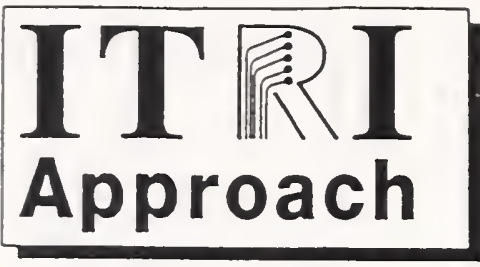

- Project Teams

- Develop project mission, define work plan, specify outcome and document work

- Once formed, teams control additions to the team and work effort

- Project management

- Majority U.S. owned

- Major R \& D resources in the U.S.

- Committed to making the required financial contribution in cash or in kind

\section{IT 展I}

\section{Operating Structure}

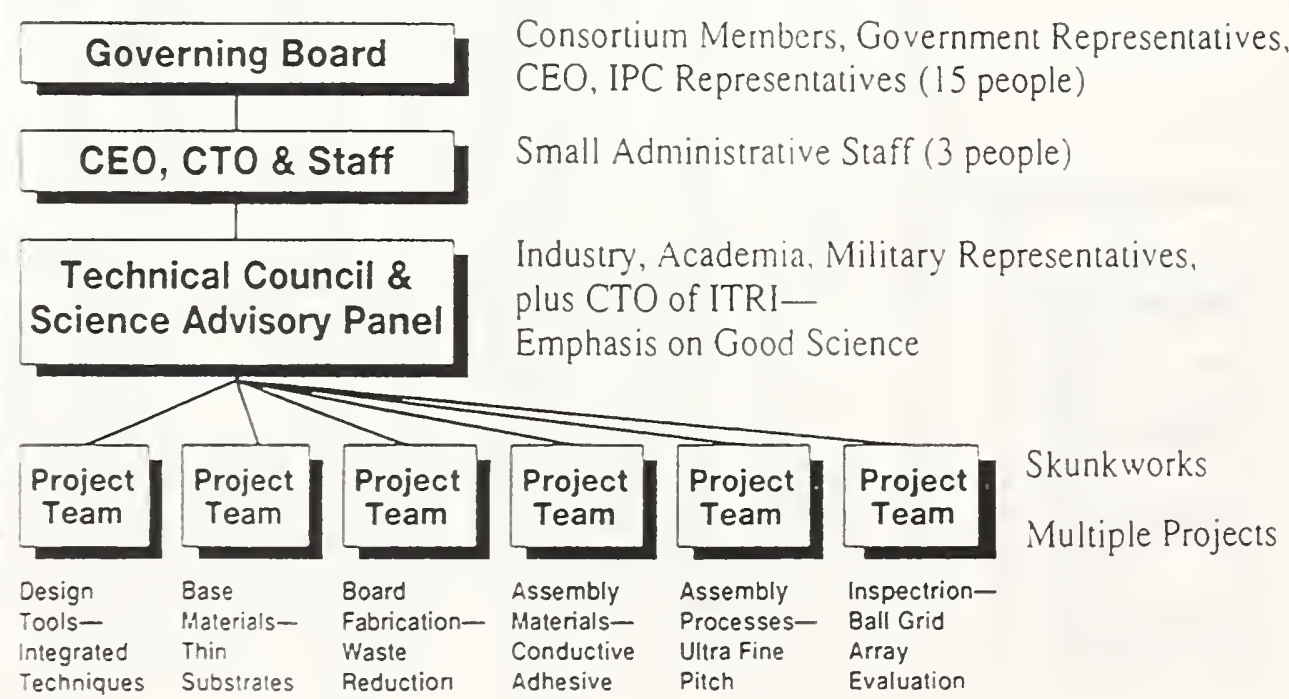

PT Leadership: Each neaded by a council member or consortium member company. PT Membership: Any member company contributing to the project. 


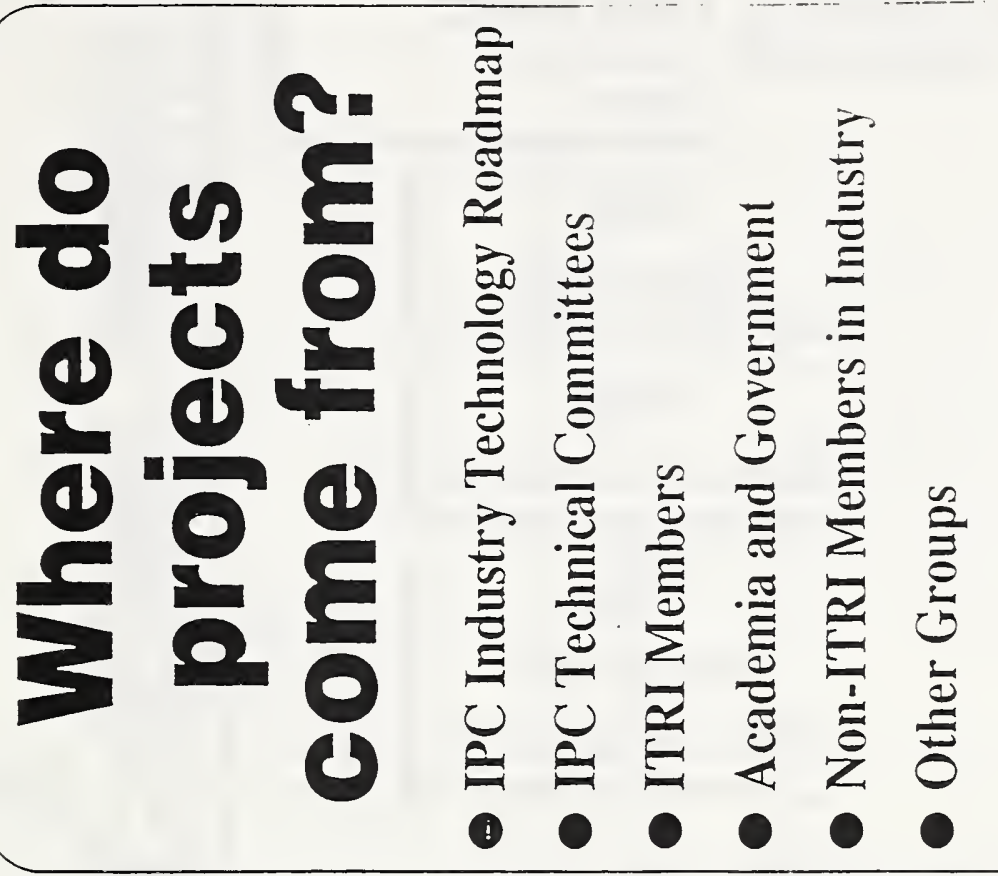

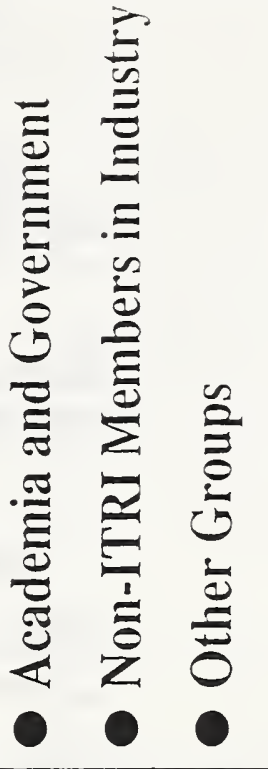

(0)

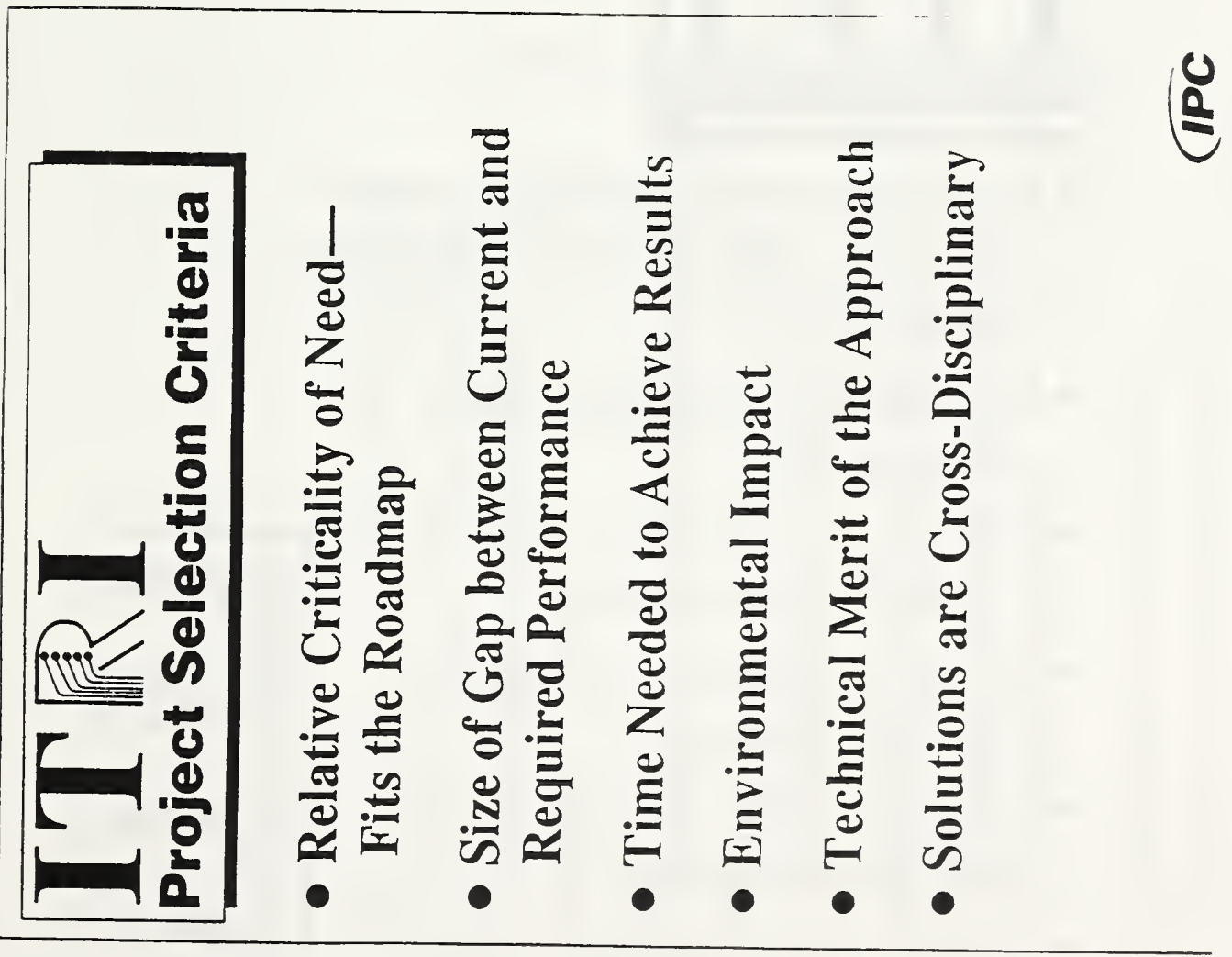




\section{Vertically Integrated Approach

\begin{tabular}{|c|c|c|}
\hline $\begin{array}{l}\text { Provide } \\
\text { Technology } \\
\text { Needs and } \\
\text { Market Pull. }\end{array}$ & \begin{tabular}{|l|l} 
IC MFRS & $\begin{array}{l}\text { PWB Users } \\
\cdot \text { Commercial } \\
\cdot \text { Military }\end{array}$ \\
\end{tabular} & $\begin{array}{l}\text { End User Systems } \\
\text { with World } \\
\text { Class Quality } \\
\text { Electronic }\end{array}$ \\
\hline & $\begin{array}{c}\text { PWB } \\
\text { Assembly }\end{array}$ & \\
\hline $\begin{array}{l}\text { fine } \\
\text { anufacturing } \\
\text { eds. }\end{array}$ & $\begin{array}{c}\text { PWB } \\
\text { Manufacturers }\end{array}$ & $\begin{array}{l}\text { Process } \\
\text { Improvements. }\end{array}$ \\
\hline $\begin{array}{l}\text { velop Next } \\
\text { neration }\end{array}$ & $\begin{array}{c}\text { PWB } \\
\text { Suppliers } \\
\text { Infrastructure }\end{array}$ & $\begin{array}{l}\text { Provide Controllable, } \\
\text { Cost Effective, } \\
\text { Environmentally } \\
\text { Benign Materials } \\
\text { \& Equipment. IPC }\end{array}$ \\
\hline
\end{tabular}

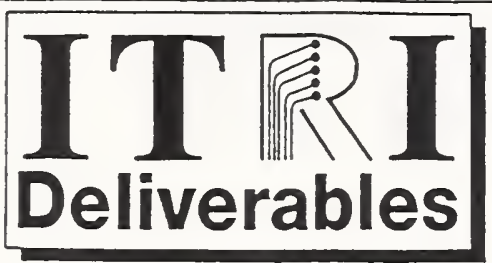

- Legal structure for sharing-antitrust protection under National Cooperative Research Act

- Development of common needs of the industry

- Survival/growth a key segment of the U.S. electronics industry

- Solutions to industry problemstoday and tomorrow

- Opportunity for consortia project participation/leadership

- Reduced costs in manufacturing

- Reduced cost, higher ROI on industry $R \& D$ 

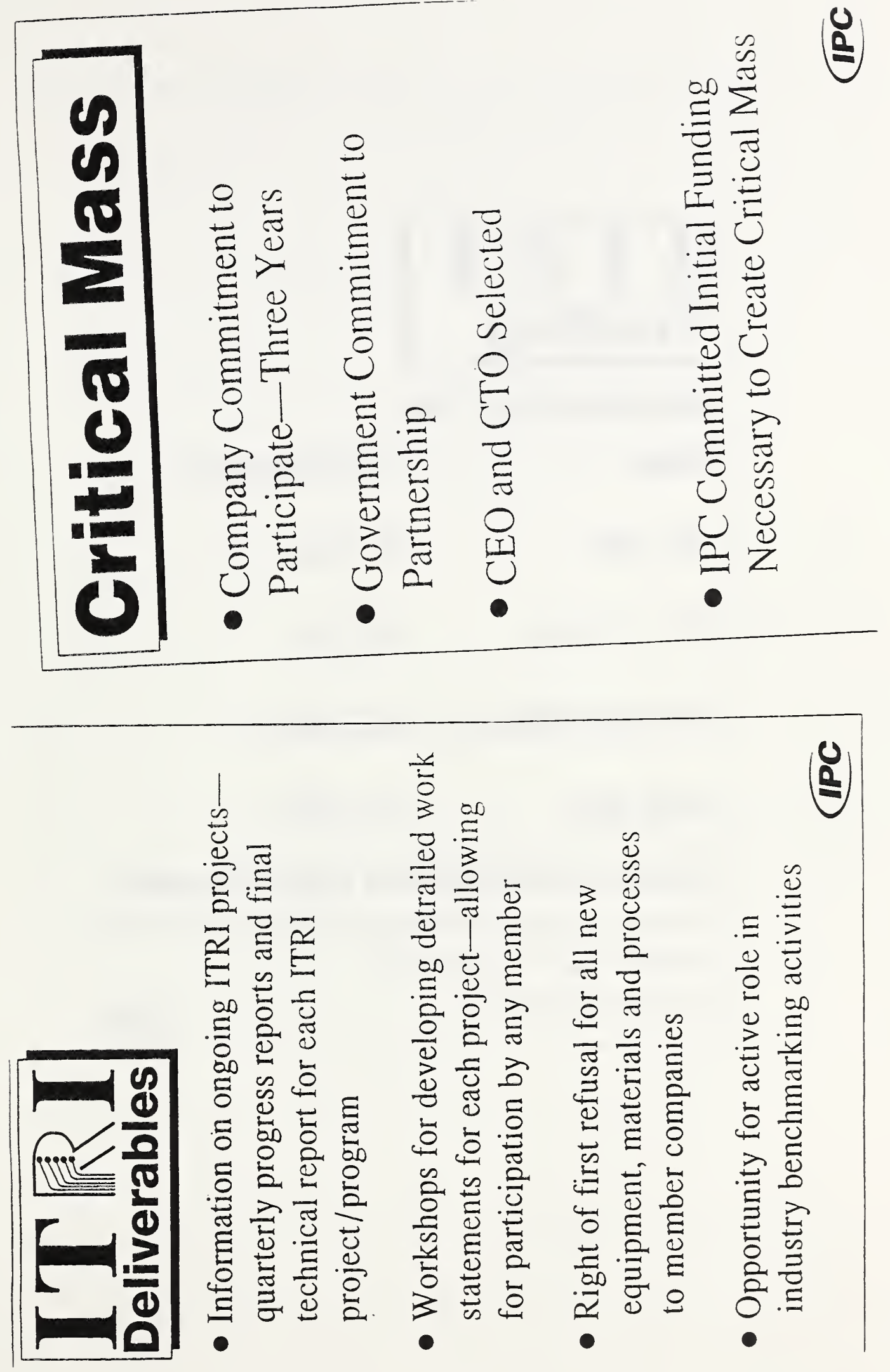


\begin{tabular}{|c|c|}
\hline \multicolumn{2}{|c|}{ Membership Fee } \\
\hline Sales & Contributions \\
\hline$<\$ 5 \mathrm{MM}$ & $\$ 1,000$ \\
\hline$\$ 5-10 \mathrm{MM}$ & $\$ 2,500$ \\
\hline$\$ 10-50 \mathrm{MM}$ & $\$ 5,000$ \\
\hline$>\$ 50 \mathrm{MM}$ & $\$ 10,000$ \\
\hline \multicolumn{2}{|c|}{$\begin{array}{l}\text { Covers Overhead and Administration } \\
\text { Private or Joint Government/Private Funding } \\
\text { of Individual Consortium } \\
\text { Projects/Programs }\end{array}$} \\
\hline
\end{tabular}




\section{Michael Schen, NIST}




\title{
TECHNOLOGY PARTNERSHIPS WITH NIST
}

\author{
Michael A. Schen \\ Materials Science and Engineering Laboratory \\ National Institute of Standards and Technology \\ Gaithersburg, MD 20899 \\ for the \\ Workshop on \\ Materials Metrology and Data for Commercial Electrical and \\ Optical Packaging and Interconnection Technologies \\ Hilton Hotel. Gaithersburg MD \\ May 6. 1994
}

\section{NIST R\&D PROGRAM IN ELECTRONIC PACKAGING AND INTERCONNECTION MATERIALS}

- May 1990 Workshop crystallized NIST R\&D planning for expanded program in electronic packaging and interconnection.

- Focus on metrology for measurements of properties of materials and materials structures.

- Well aligned with NIST's historic role

- Supports oft-neglected national infrastructure

- Generic precompetitive nature

- Broadly applicable across the industry

- Supplements Prior Efforts in Design and Manufacturability of Packaging Systems (Thermal Control, Wire Bonding, Hermeticity,

Electromigration) 


\section{NIST R\&D PROGRAM}

- Program Focus:

packaging issues at and above the chip level

- Program Scope:

processing, inspection methods, properties data, performance measurements, and improved materials and tests for... substrates, encapsulants, adhesives, solders, conductors, and interconnects

- Program Goals:

develop techniques and procedures for making in-situ measurements on materials and structures having micrometer and sub-micrometer dimensions

quantify and record the divergence of material properties from their bulk values as dimensions are reduced and structures are confined

standardize measurements on materials and material structures

disseminate materials properties data in a form that can be used by the microelectronics industry

\section{PROJECTS IN PACKAGING AND INTERCONNECTION}

Computerized Information Exchange via STEP, Joe Carpenter (301.975.6397) developing and implementing standard's for the computerized exchange of digital data consistent with the Intemational Organization for Siandardization (ISO) program called STEP (STandard for the Exchange of Product data modell for electronic packaging materials

Micromechanical Measurements, David Read (303.497.3853) developing micromechanical measurement techniques for the measurement of elastic modulus, yield and ultimate strength, and elongation to failure of metal thin films and developing experimental mechanics techniques to verify analytical predictions by direct observation of local displacements, strains and stresses

Dimensional Stability of Polymers, Michael Schen (301.975.6741)

joint development of measurement techniques, protocols, and standard reference materials with electronics packaging materials suppliers and users for coefficient of thermal expansion (CTE) and dimensional change due to absordtion of moisture

Cure Monitoring in Electronic Packaging. Tom Davis (301.975.6728)

developing means of assessing performance of polymer films and layers by developing methods of measuring the conversion of polymer precursors io final layers and developing methods for measuring influence of cure on polymer properties most significant to electrical performance and intertacial integrity

Polymer Process Monitoring, Fred Mopsik (301.975.6747)

develooing dielectric and capacitance methodologies for polymers as processing and inspection methods. for groperties data, Derformance measuremenis and improved materials for the substrates, encapsulan:s. and conductors used in electronic packaging and interconnects 
PROJECTS (continued)

Mechanism of Adhesion of Polymers, Wen-Li Wu (301.975.6839)

developing a fundamental understanding of the parameters important in interface debonding and the role of environmental agents, such as water, on the interfacial stability of thin polymer fitms used in

packaging on an Angstrom scale using neutron and $x$-ray reflectivity

Self-Reinforced Packaging Composites, Barry Bauer (301.975.6849)

developing and applying concepts in advanced interpenetrating polymer networks to the synthesis and characterization of self-reinforced polymeric resins with improved thermel, mechenical, and dimensional control

Electromagnetic Characterization of Electronic Interconnects, Roger Marks (303.497.3037)

developing methods to electrically characterize interconnections by measuning very high frequency (GHz) transmission line properties, impedances, and scattering parameters using microwave instrumentation and seeks to define the fundamental parameters and procedures for conducting accurate measurements

Solderability Measurements and Optimization, John Manning (301.975.6157) developing new messurement techniques for solderability based on improved understanding of surface wertability and reactions at solder-substrate interfaces leading to new accelerated tests for determining component solderability after tinning

Lead-free Solders, Carol Handwerker (301.975.6158)

developing tools for understanding and evaluating solderability, determining phase diagrams, and measuring the mechanical properties of environmentally safe alternatives for lead-based solder alloys in conjunction with the National Center for Manufacturing Sciences (NCMS)

\section{NIST Proposal: THE ADVANCED PACKAGING AND INTERCONNECTION MATERIALS METROLOGY FORUM}

- A NIST-wide forum for building industry collaborations and broadly leveraging NIST results within the U.S. electronic packaging and interconnection and material supplier industries

- Help bridge the gap between material suppliers and microelectronic device manufacturers and users

- Focus on improvements in materials measurement science and data quality / availability to support goals in commercial microelectronic packaging established by U.S. industry

- Vehicle for accelerating development, standardization, and implementation of improved metrology

- Utilize CRADAs to protect intellectual property and assign rights 
JOHN GUDAS, NIST 


\title{
Nढ़ा \\ Advanced Technology Program
}

\author{
John P. Gudas
}

Technology Administration

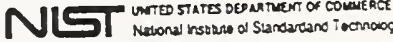

DR. JOHN P. GUDAS

Department of Commerce

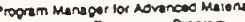

Norancoso Tecroosey Propram

\section{THE FEDERAL ROLE IN TECHNOLOGY}

- How is the government investing in science and technology?

- Total U.S. R\&D > \$150 B / year

- Federal government provides $45 \%$ of the funds for U.S. R\&D

»60\% for defense, mostly weapons system development

" $15 \%$ for health

" $0.5 \%$ for industrial development

-What has changed?

- Global competition has accelerated the rate of innovation and challenged U.S. industry

- Post Cold War $\Rightarrow$ opportunity to refine our technology investment strategy 


\section{THE FEDERAL ROLE IN TECHNOLOGY}

- How is the government responding to change?

- Invest in civilian technology

- Drive defense toward a dual-use technology base

- Transfer technology from government labs

- Continue commitment to basic science

\section{NIST MISSION}

- To promote U.S. economic growth by working with industry to develop and apply technology, measurements, and standards. 


\section{NIST OUTYEAR SUMMARY}

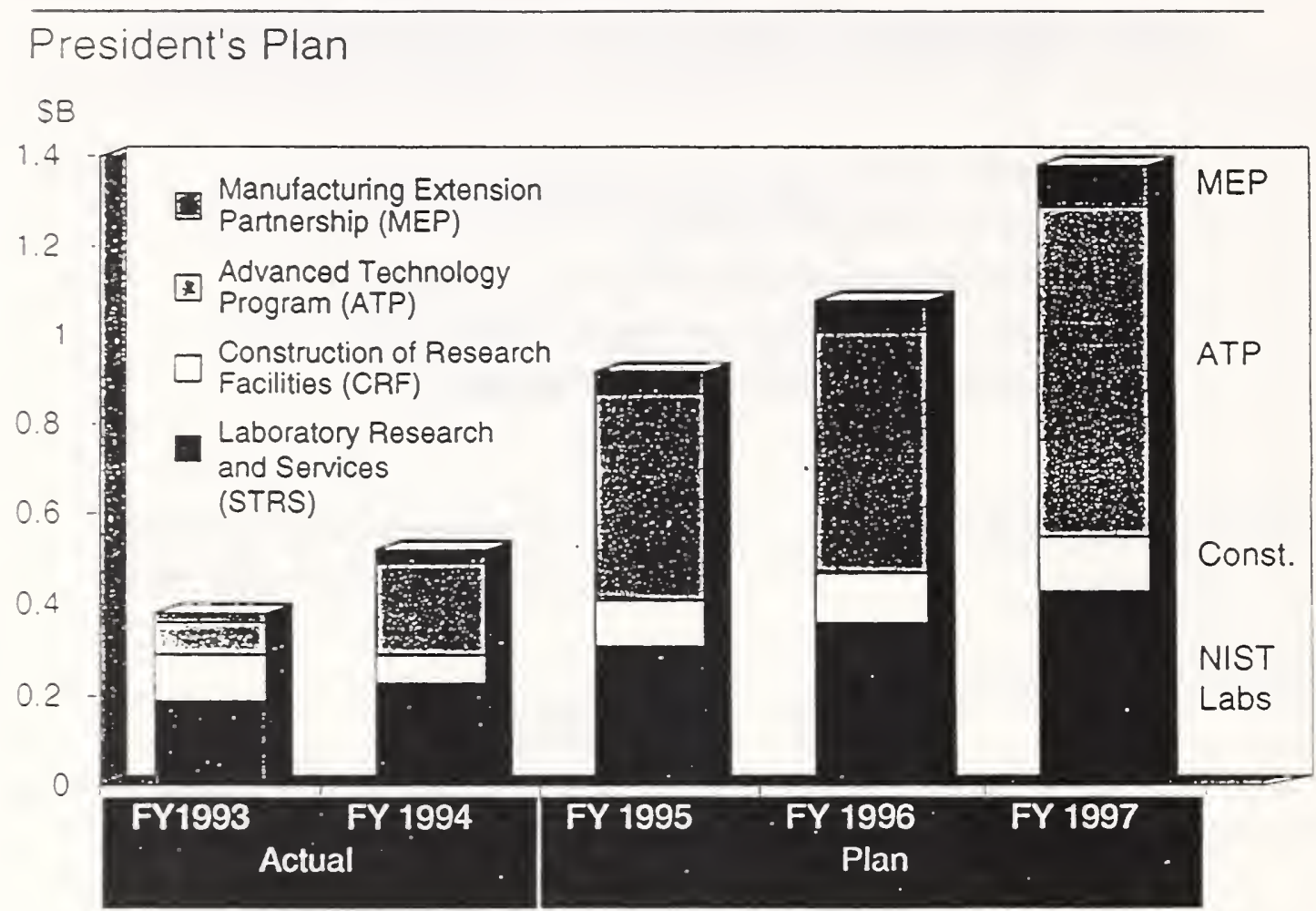

\section{ATP MISSION}

- Stimulate U.S. economic growth by developing high-risk and enabling technologies through programs proposed and cost shared by industry 


\section{BASIC CHARACTERISTICS}

- Unique mission focus - - high-risk enabling technology development to stimulate U.S. economic growth

- Partnership with industry -- industry conceives and proposes ideas, executes and cost-shares projects; ATP applies selection criteria

- Focused and general competitions - - for depth and breadth

- Competitive selection process -- technical and business reviews

- Sunset provisions - - for both programs and projects

- Performance metrics - - for both process and programs

\section{ATP BUDGET PROFILE}

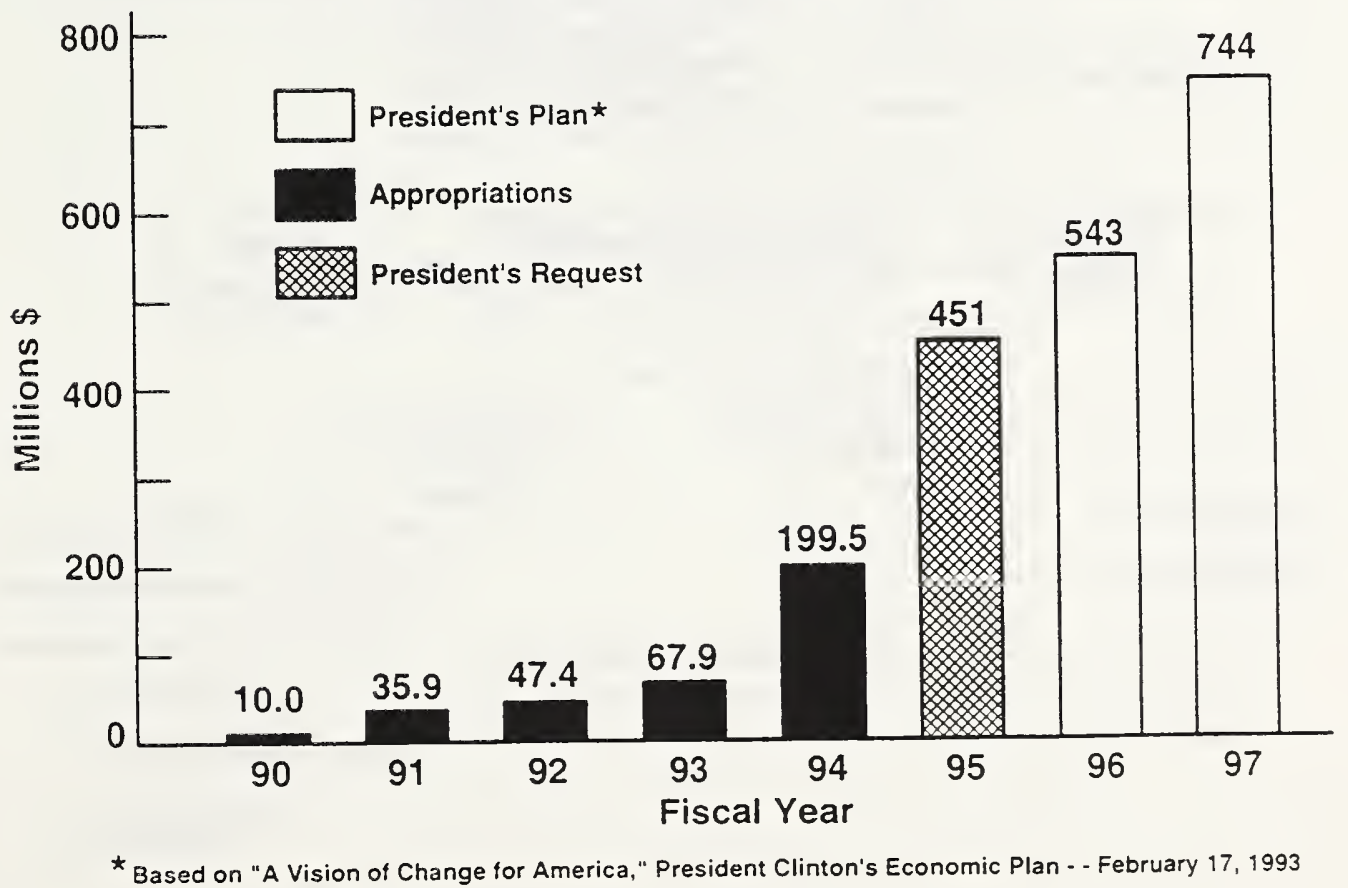


- Individual companies

- No more than 3 years

- Up to $\$ 2 M$ total

- NIST pays only direct costs

- Joint ventures

- No more than 5 years

- No limit on award amount

- NIST share less than $50 \%$

- No direct funding to universities, government agencies or non-profit independent research institutes

\section{SELECTION PROCESS MANAGEMENT}

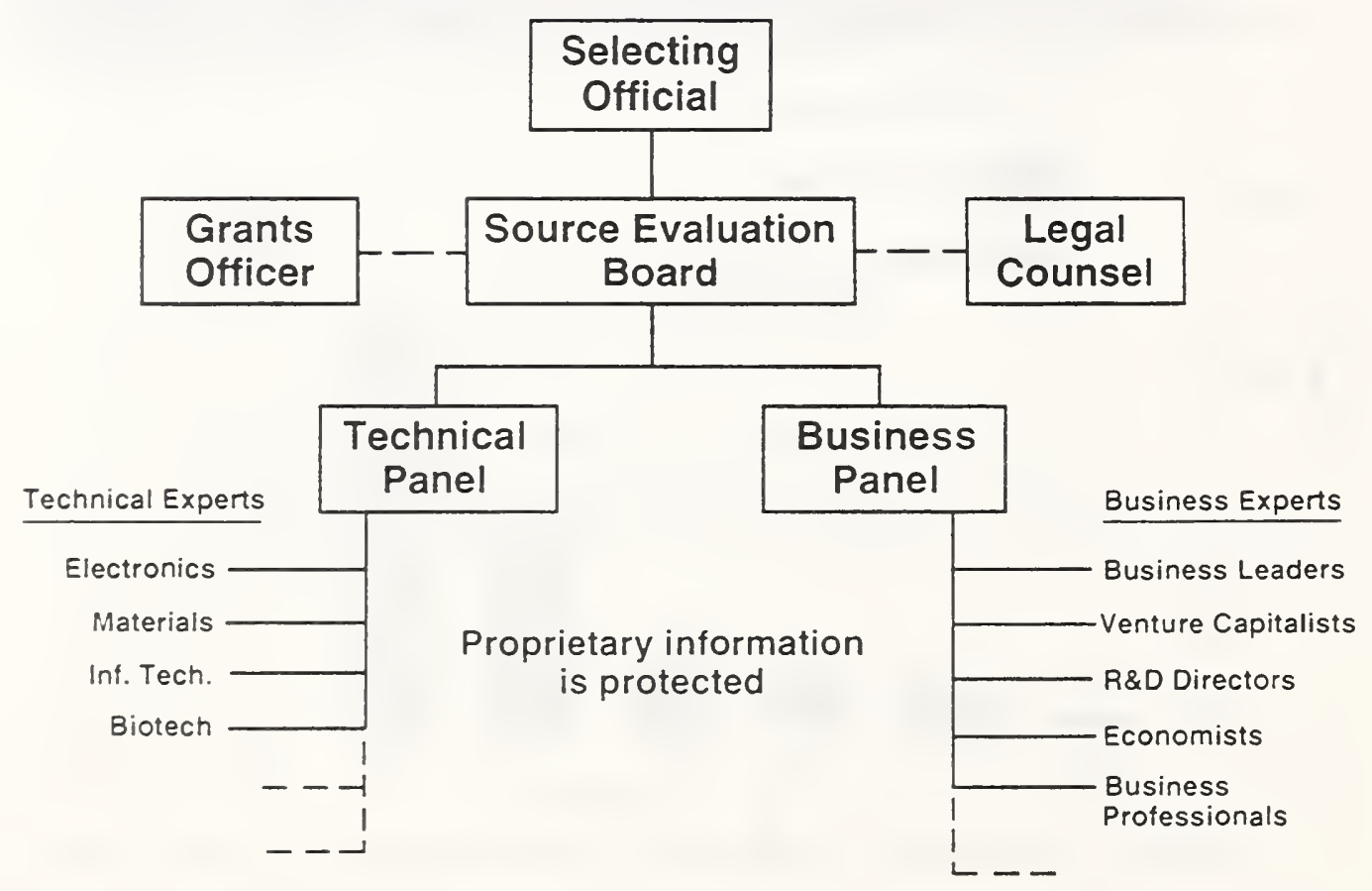




\section{ATP PROJECT SELECTION PROCESS}

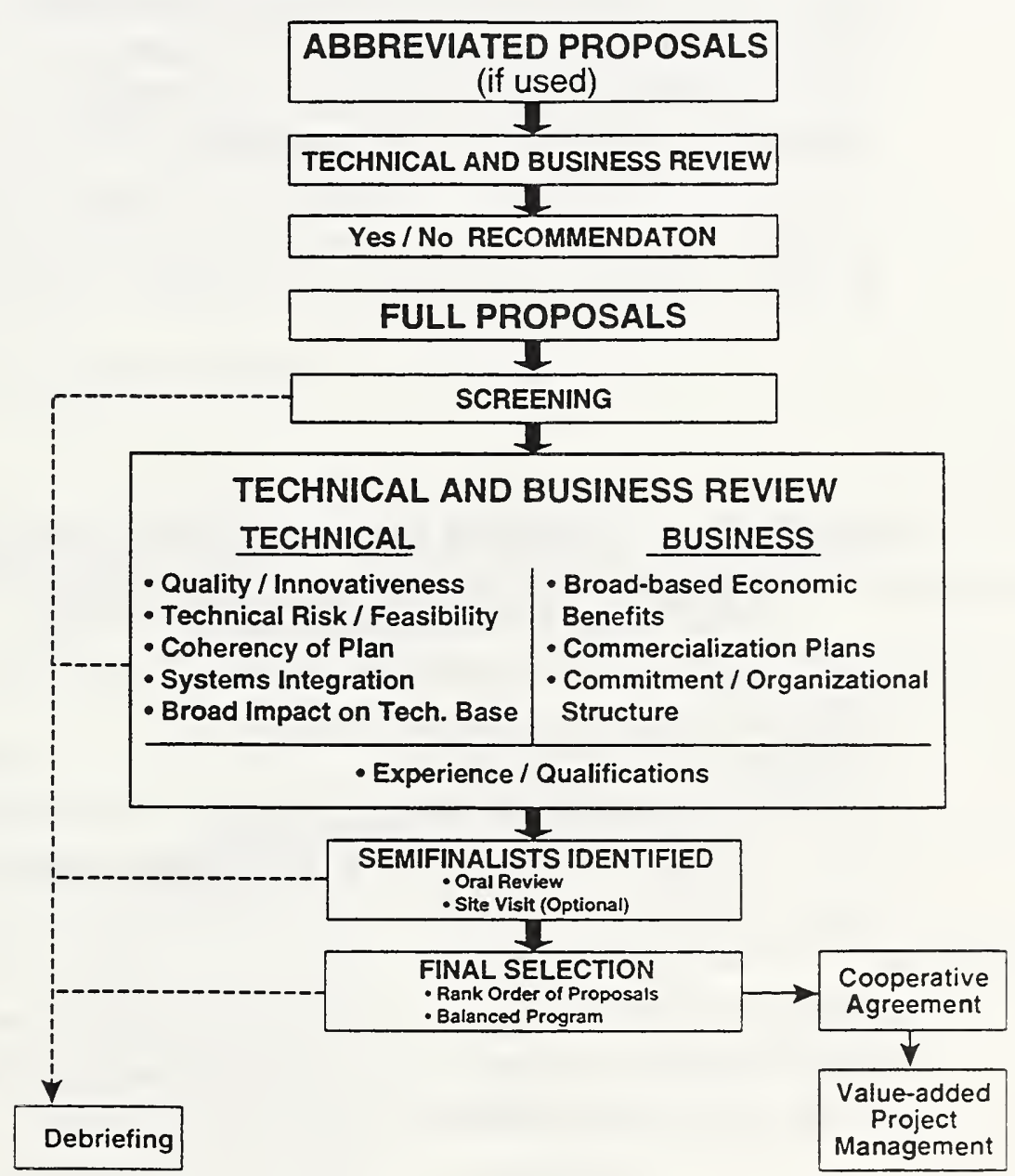




\section{INTELLECTUAL PROPERTY PROVISIONS}

- Companies incorporated in the U.S. keep intellectural property rights

- Companies can license

- Government reserves the right to a royalty-free non-exclusive license for government use

- Non-disclosure (trade secrets protected)

- Government rights rarely invoked

\section{FOREIGN COMPANY PARTICIPATION}

- Project must result in economic benefits to the U.S.

- R\&D and manufacturing in the U.S.

- Increase U.S. employment

- Promote U.S. supplier infrastructure

- U.S. - owned companies also must meet these requirements

- Country of origin must grant opportunities to U.S. - owned companies comparable to any other company in that country and protect intellectual property

- PL 102-245 authorizes suspension of award if criteria no longer satisfied 


\section{ATP STATISTICS}

Proposals Submitted

Participating Organizations*

Total ATP Funding Requested

Total Estimated Cost-Share

Number of Awards

(Joint Ventures)

(Single Applicants)

Participating Organizations*

Total ATP Funds Committed

Total Estimated Cost-Sharing

Award Size - - Range (ATP Funds)

* Excludes Subcontractors
912

1352

$\$ 1.5 \mathrm{~B}$

$\$ 1.5 \mathrm{~B}$

89

200

$\$ 247 \mathrm{M}$

$\$ 268 \mathrm{M}$

$\$ 500 K-\$ 20 M$

\section{ATP TECHNOLOGY AREAS}

- Machine Tools

- Image Recognition \& Processing

- Semiconductor Processing

- Genetic \& Tissue Engineering

- Flat Panel Displays

- Lasers, Optics \& Electro-optics

- High Performance Computers

- Optical Communications.

- Ceramics, Composites, \& Polymers
- Automated Mfg. \& Robotics

- Motor Vehicle Assembly

- Plastic Recycling

- Superconductors

- Energy Conservation \& Distribution

- X-ray Lithography \& Optics

- Optical \& Magnetic Storage

- Printed Wiring Boards

- Illumination 
FOUR GENERAL COMPETITIONS

\section{TECHNOLOGIES FUNDED BY ATP AS A PERCENT OF $\$ 247 \mathrm{M}$ AWARDED}

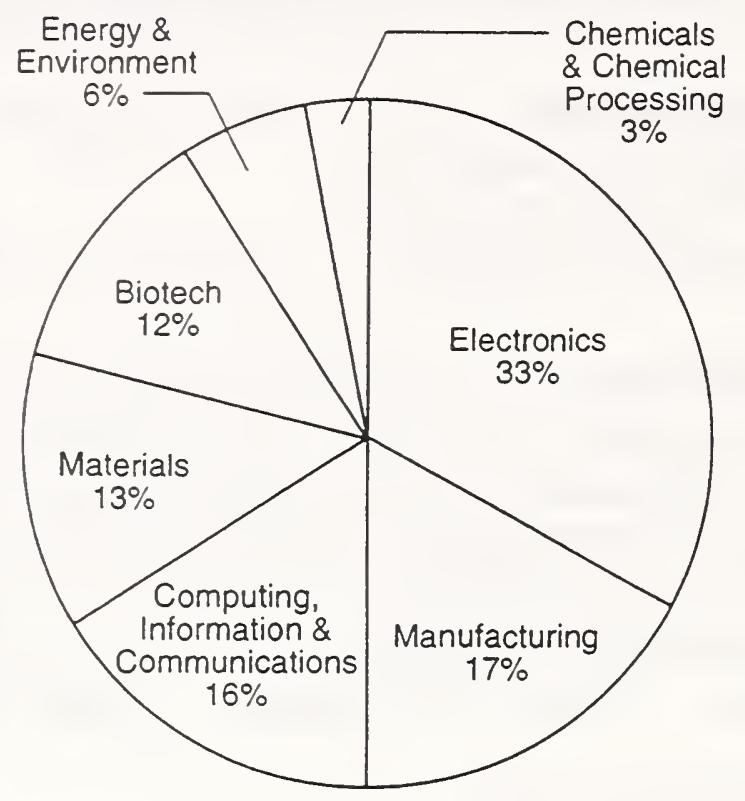

FOUR GENERAL COMPETITIONS

\section{\$247 MILLION OF ATP FUNDS AWARDED BY TYPE OF ORGANIZATION}

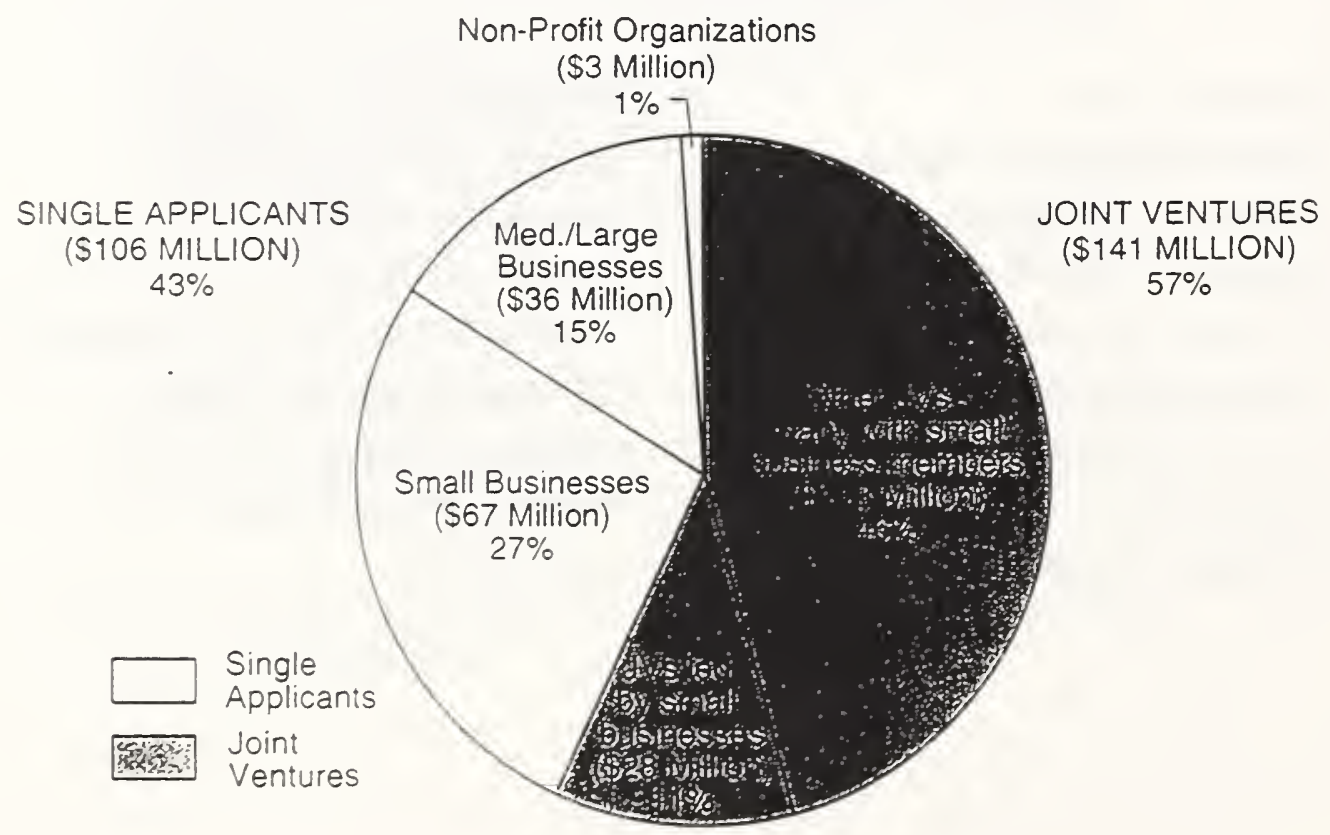


FOUR GENERAL COMPETITIONS

\section{ATP 89 AWARDEES} BY TYPE OF ORGANIZATION
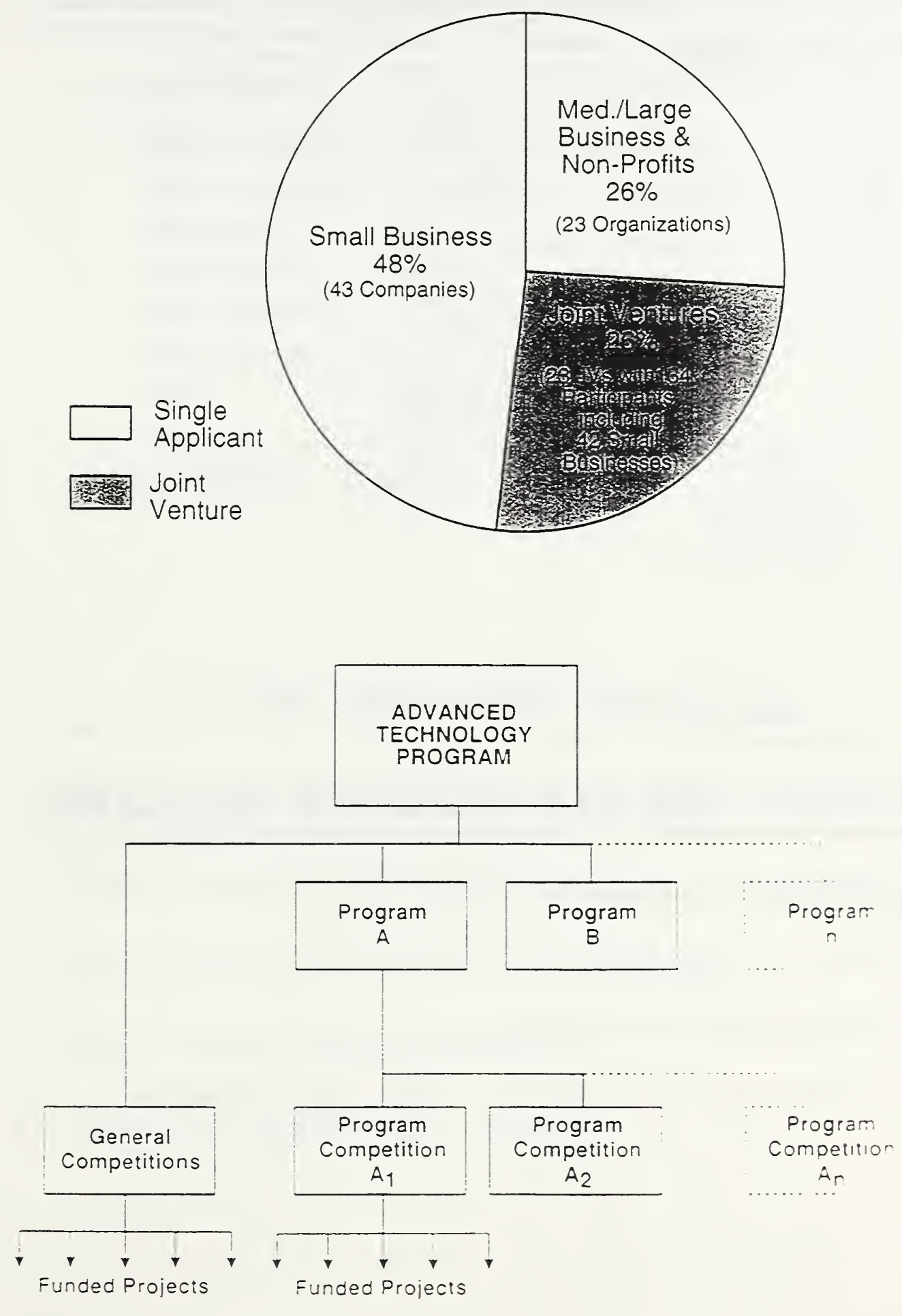


\section{PROGRAM PLANNING, SELECTION AND EXECUTION}

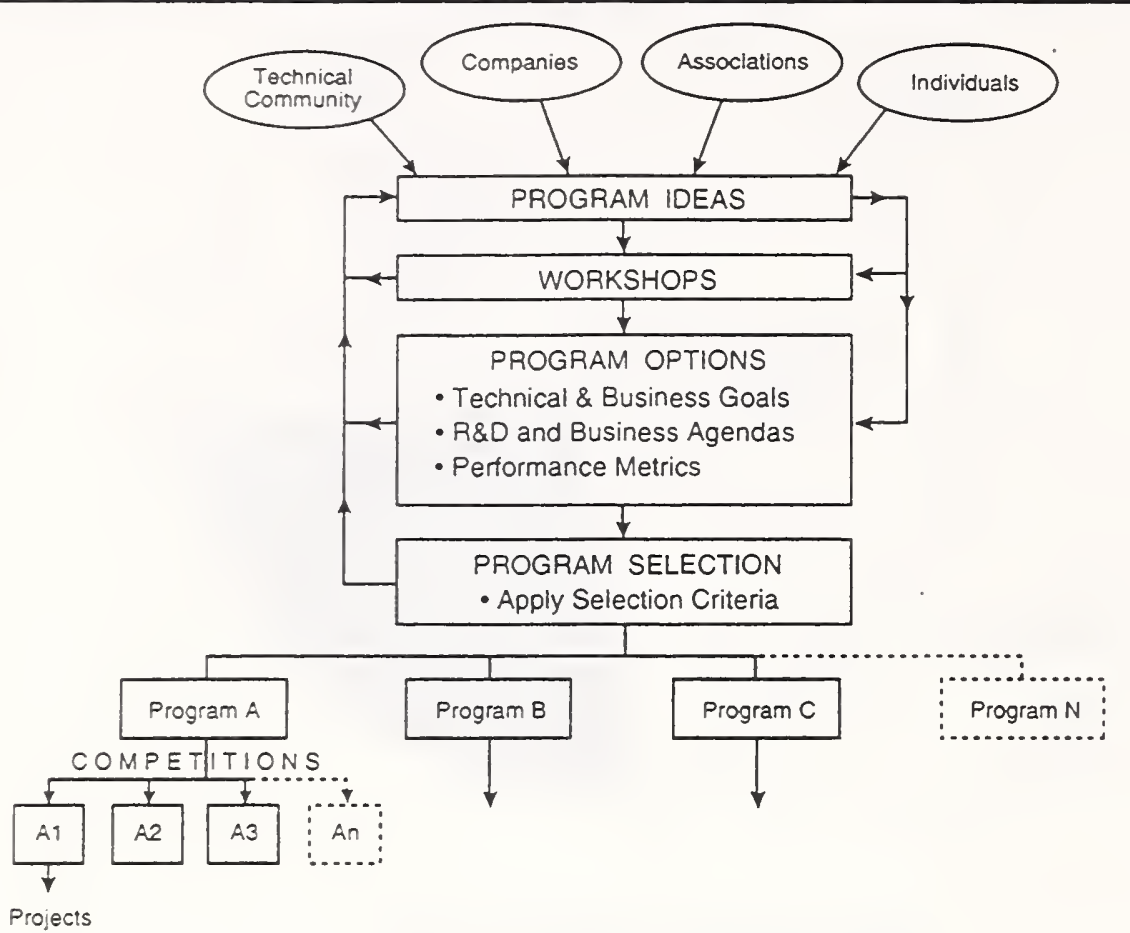

\section{CRITERIA FOR ATP PROGRAM SELECTION}

1. Potential U.S. economic benefit

2. Good technical ideas

3. Strong industry commitment

4. Opportunity for ATP funding to make a major difference 


\section{PROGRAM IDEAS RECEIVED}

(Through February 1994)

- 550 submissions - - ideas still coming in

$\begin{array}{lrll}\text { Biotechnology } & 51 & \text { Chemical } & 18 \\ \text { Communications } & 31 & \text { Construction } & 12 \\ \text { Electronics } & 64 & \text { Energy } & 29 \\ \text { Environment } & 25 & \text { Food } & 12 \\ \text { Instrumentation } & 10 & \text { Manufacturing } & 54 \\ \text { Mass Storage } & 7 & \text { Materials } & 68 \\ \text { Optics } & 15 & \text { Packaging } & 21 \\ \text { Services } & 14 & \text { Software } & 73 \\ \text { Transportation } & 29 & \text { Other } & 17\end{array}$

\section{FY 1994 FOCUSED PROGRAMS}

-94-02 Manufacturing Composites Structures

-94-03 Computer-integrated Manufacturing for Electronics

-94-04 Healthcare Information Infrastructure

-94-05 Tools for DNA Disgnostics

-94-06 Component-based Software 


\section{ATP MEASURES OF SUCCESS}

Longer Term Measures:

- Rates of Return on Investment (Private, Public, and Social)

- Aggregate Employment Effects

- Sales and Value added

- Manufacturing Costs / Product Quality / Time to Market

- Market Share (U.S. and International)

- New Industry Creation

- Multiple-Use Technology Creation

\section{Contacts:}

\section{ADVANCED TECHNOLOGY PROGRAM}

email: atp@ micf.nist.gov Telephone: 1-800-ATP-FUND

$(1-800-287-3863)$

Facsimile: (301) 926-9524 
Nicholas NACLERIo, ARPA 


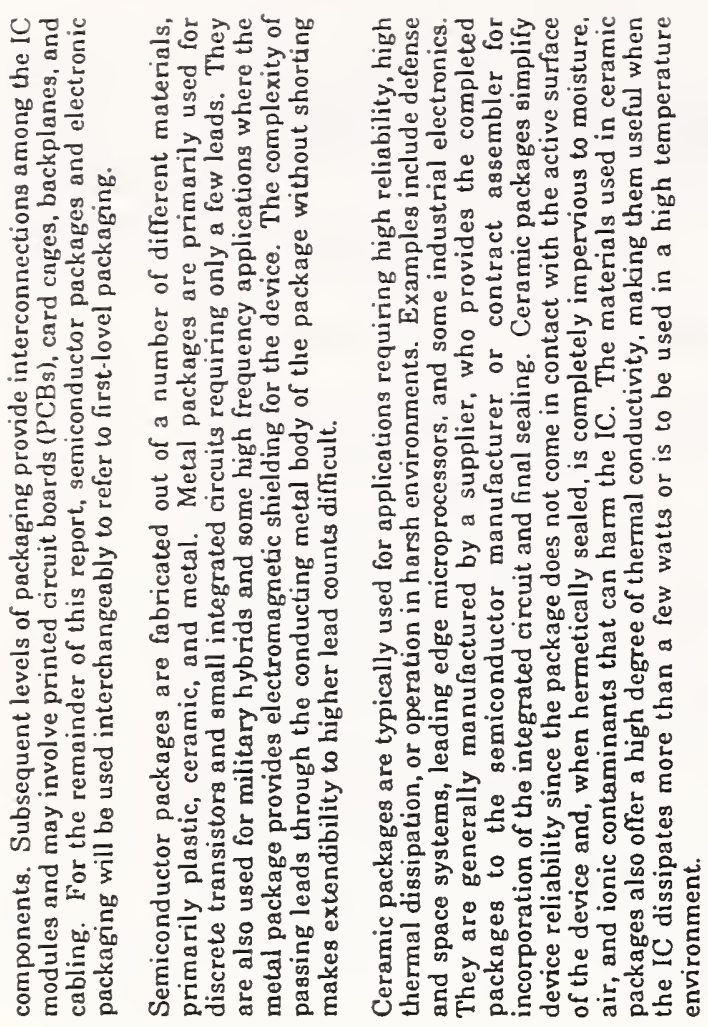

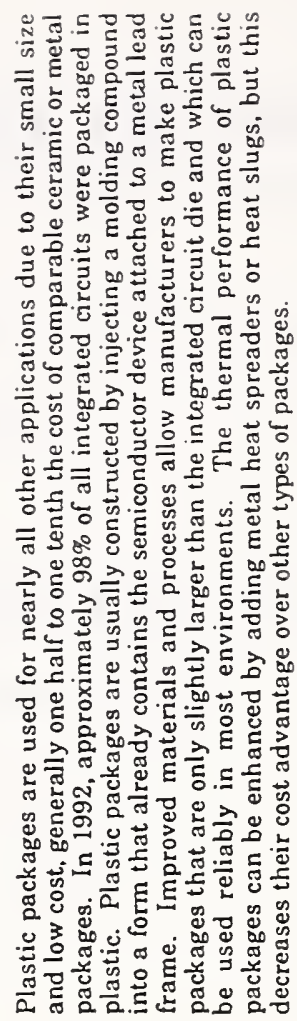

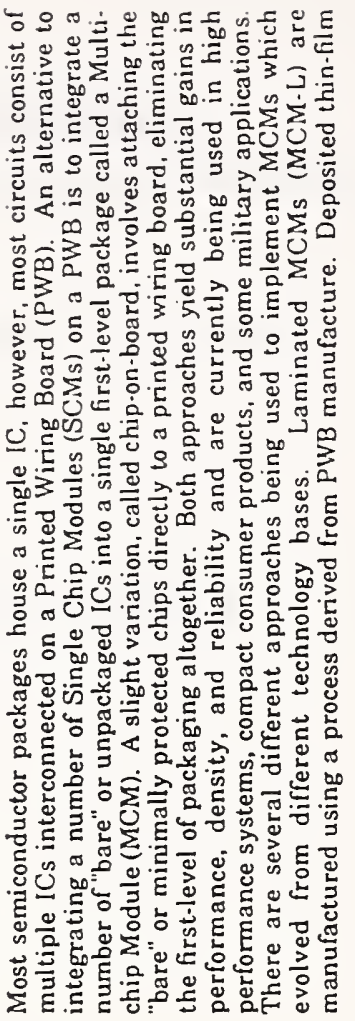

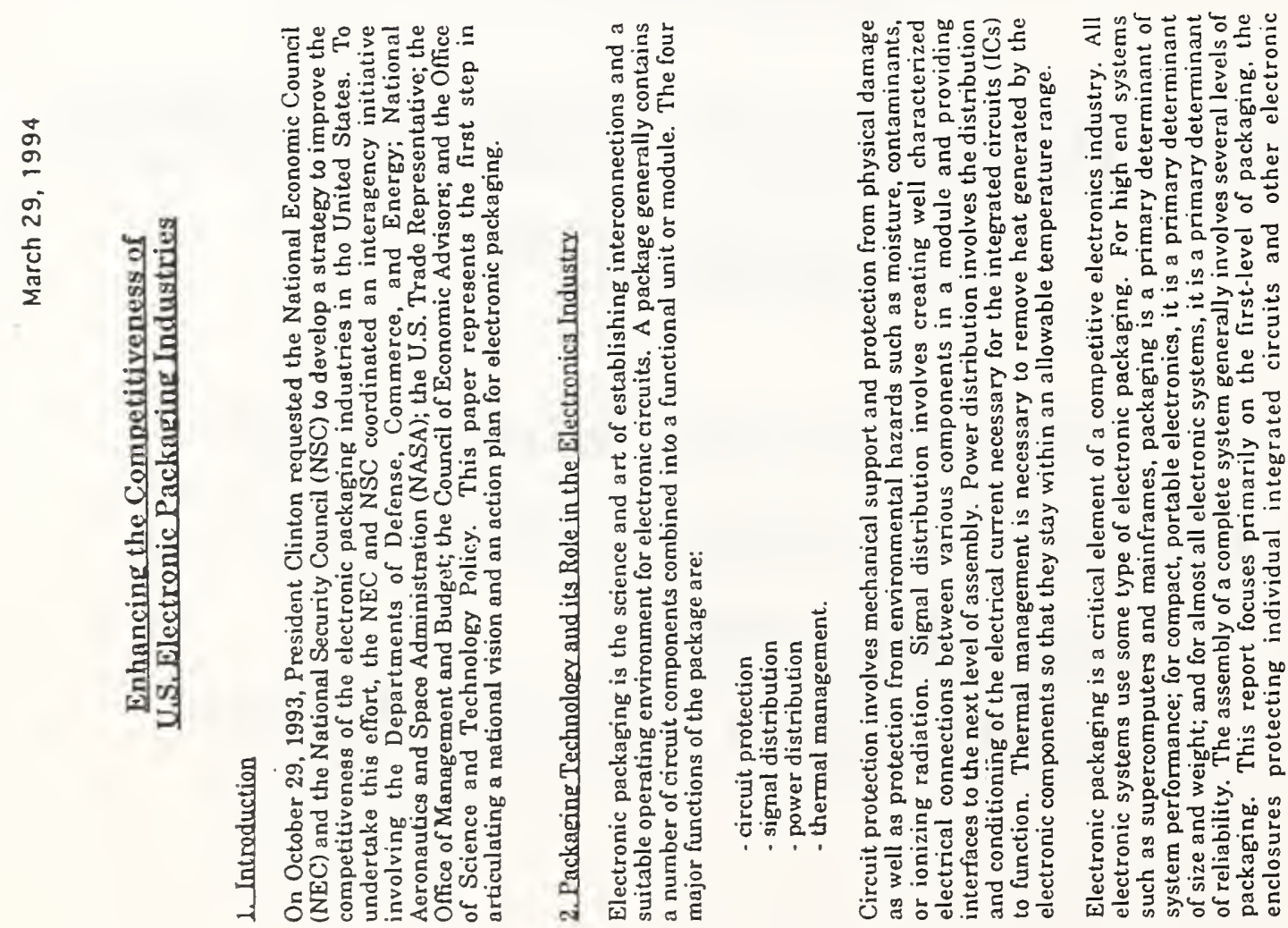




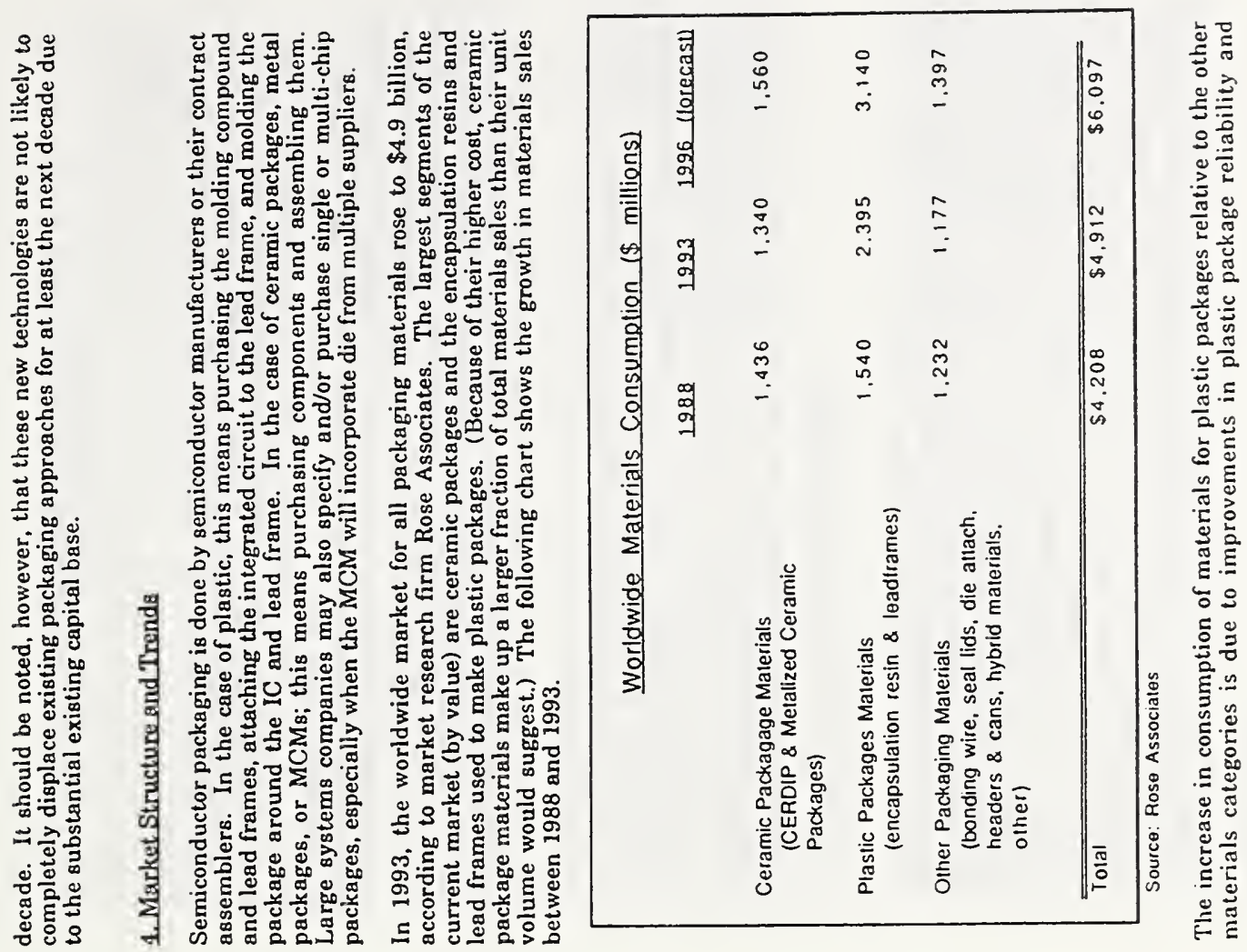

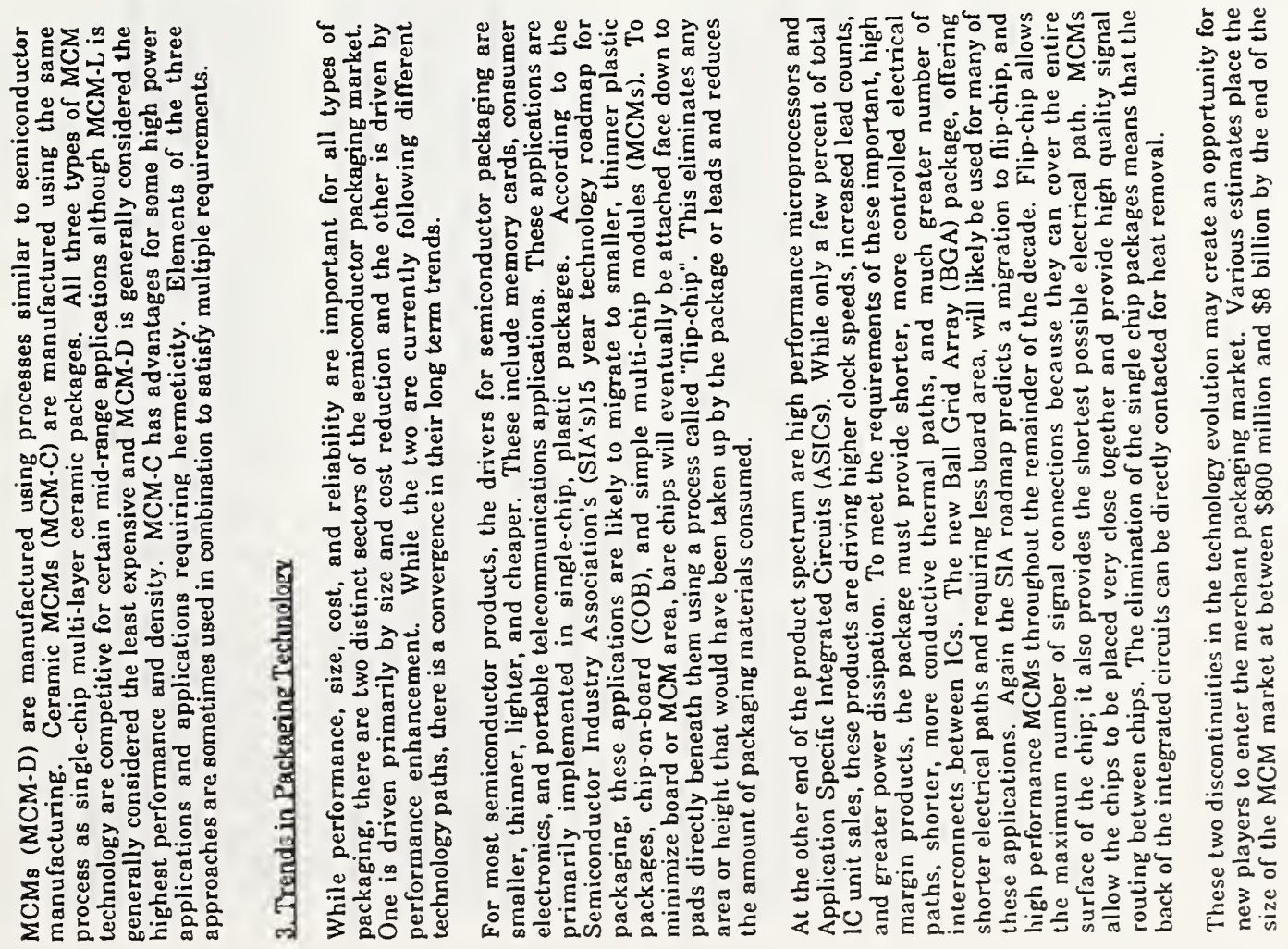



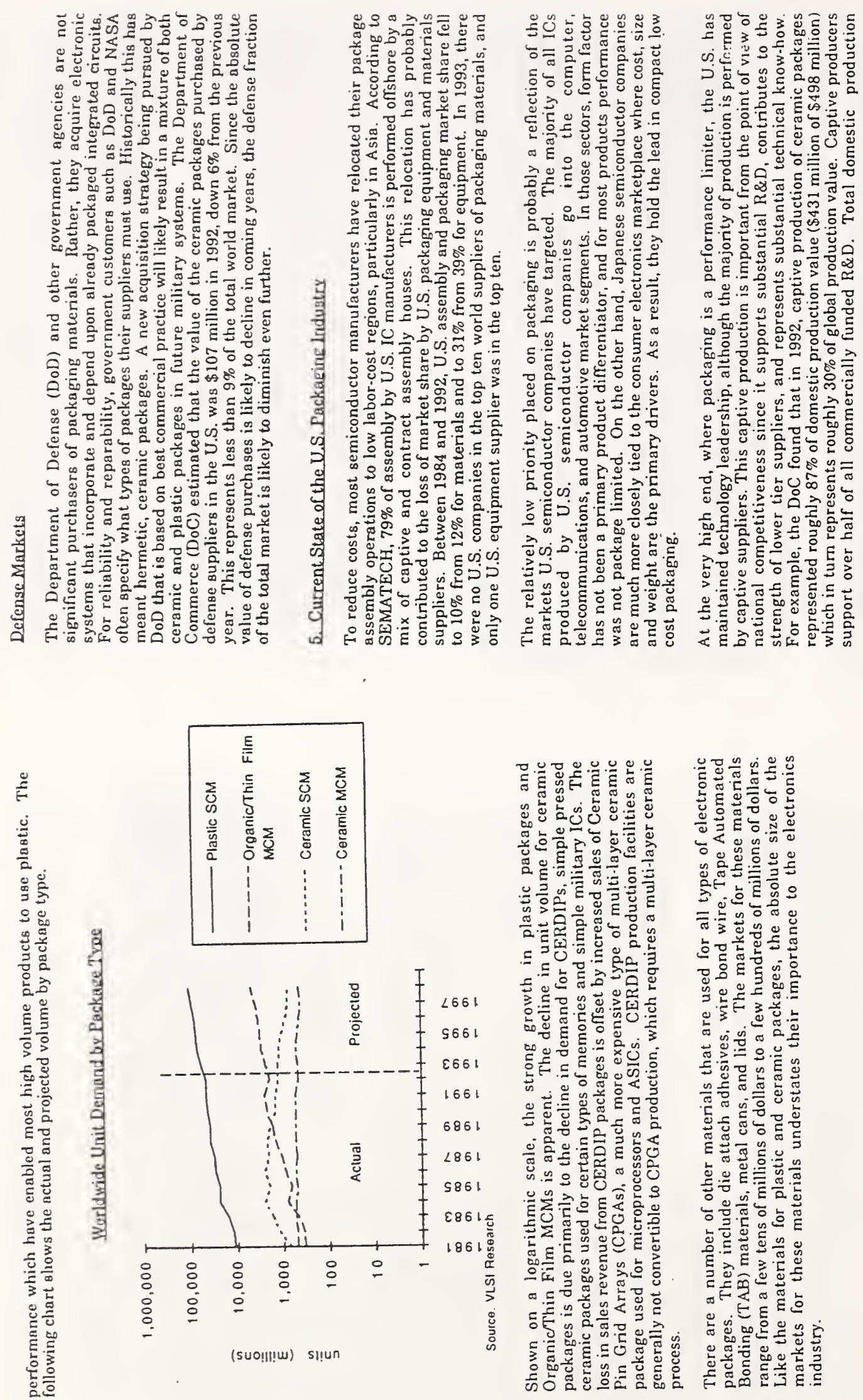

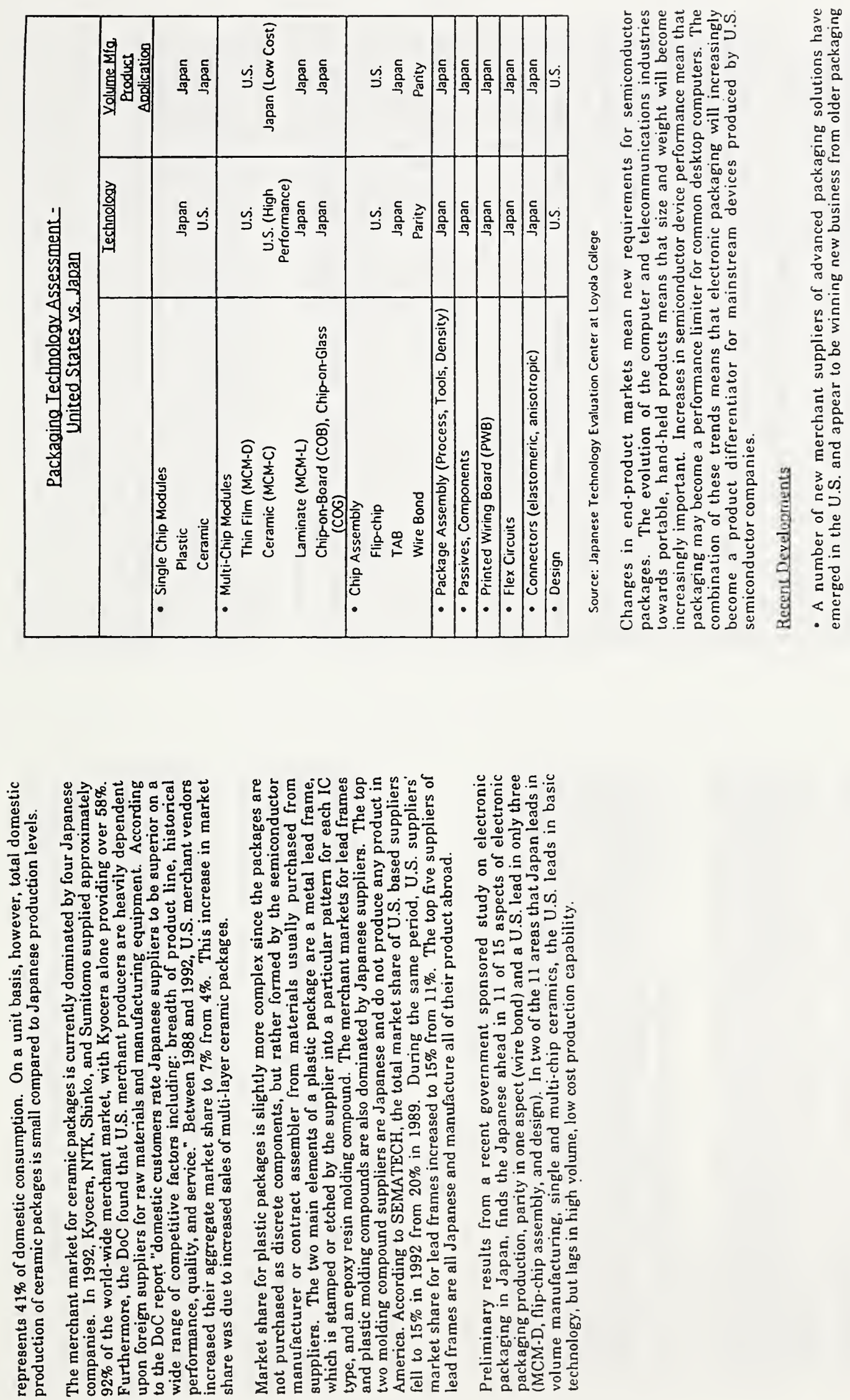

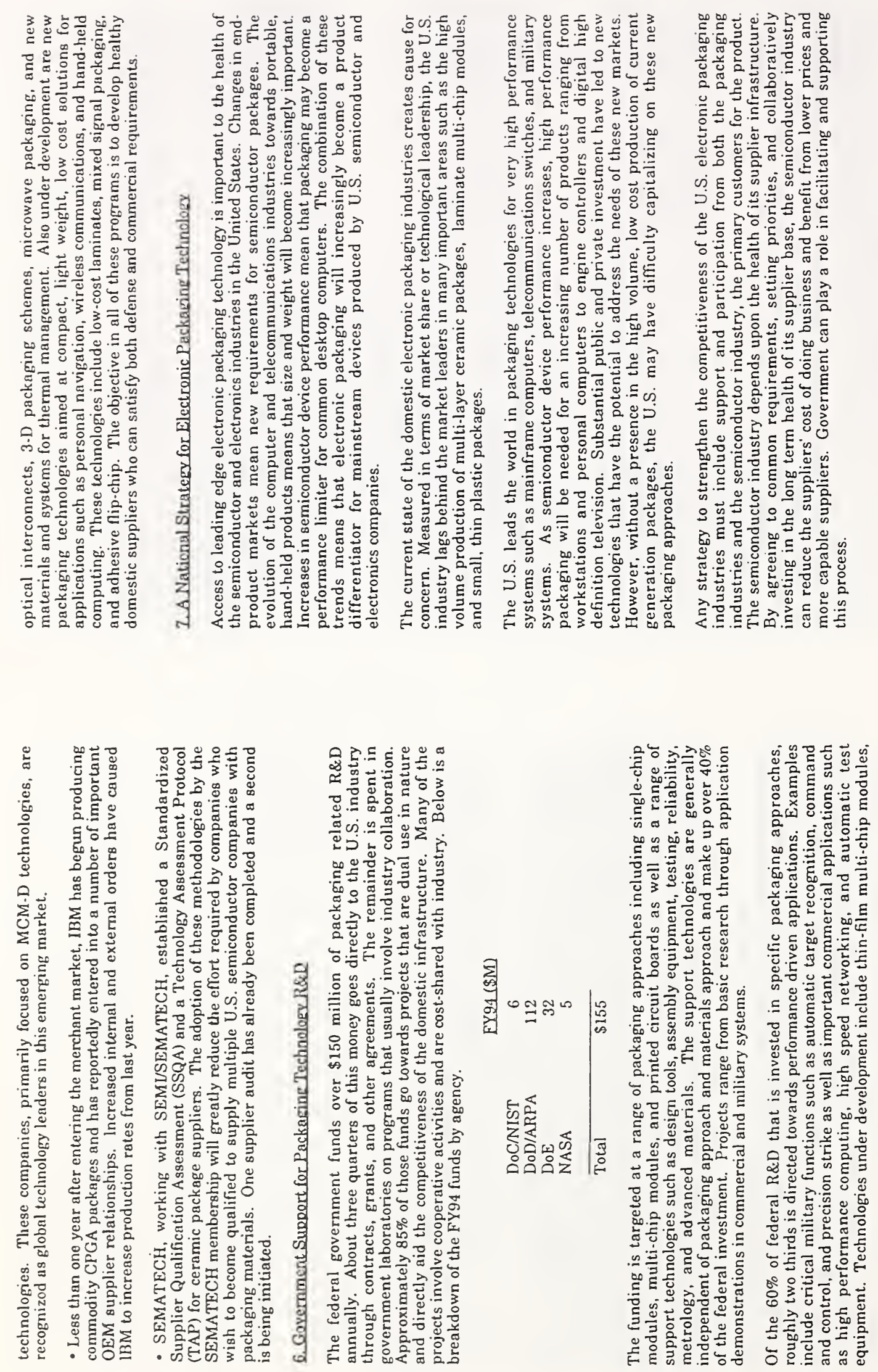


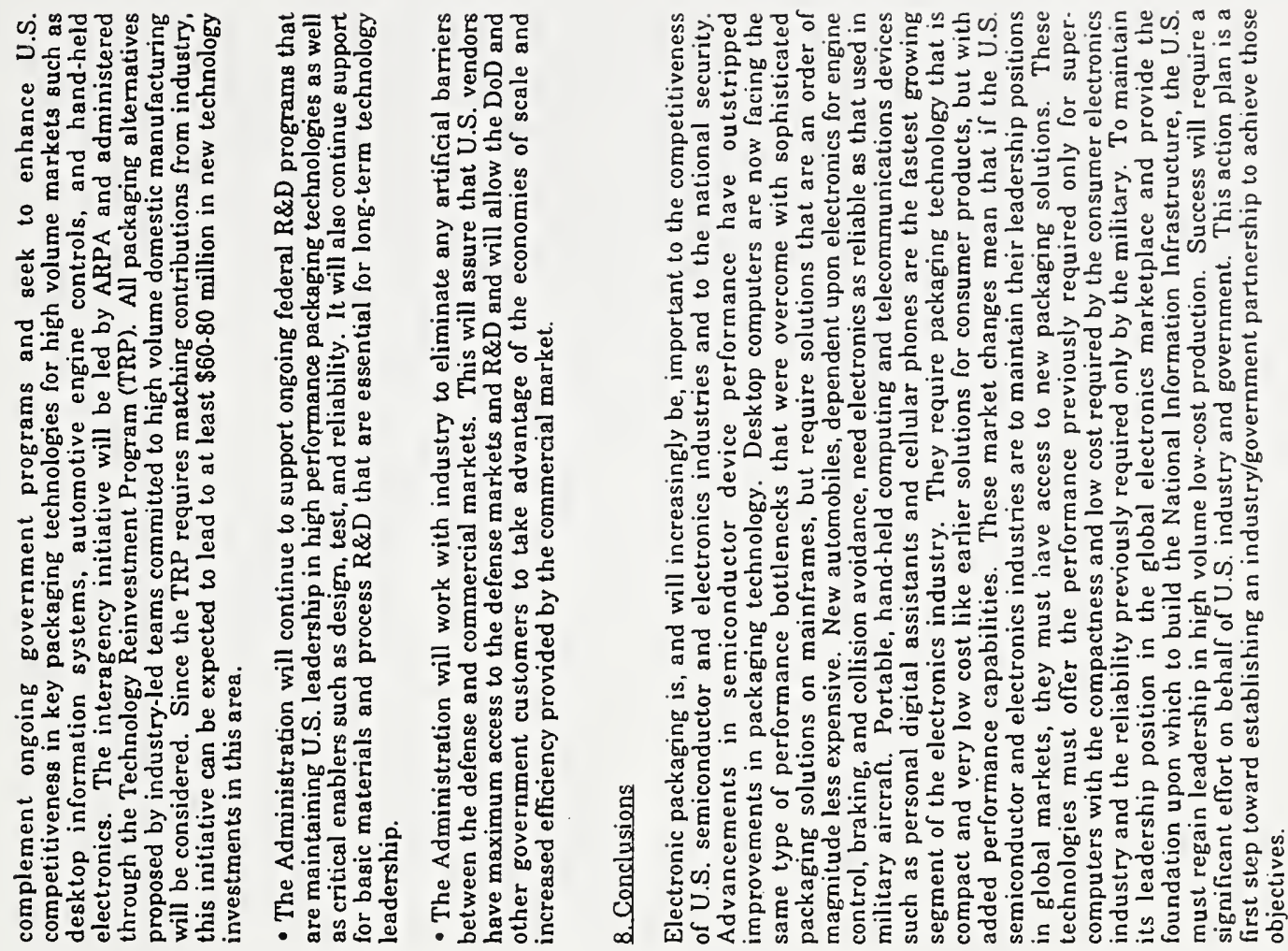

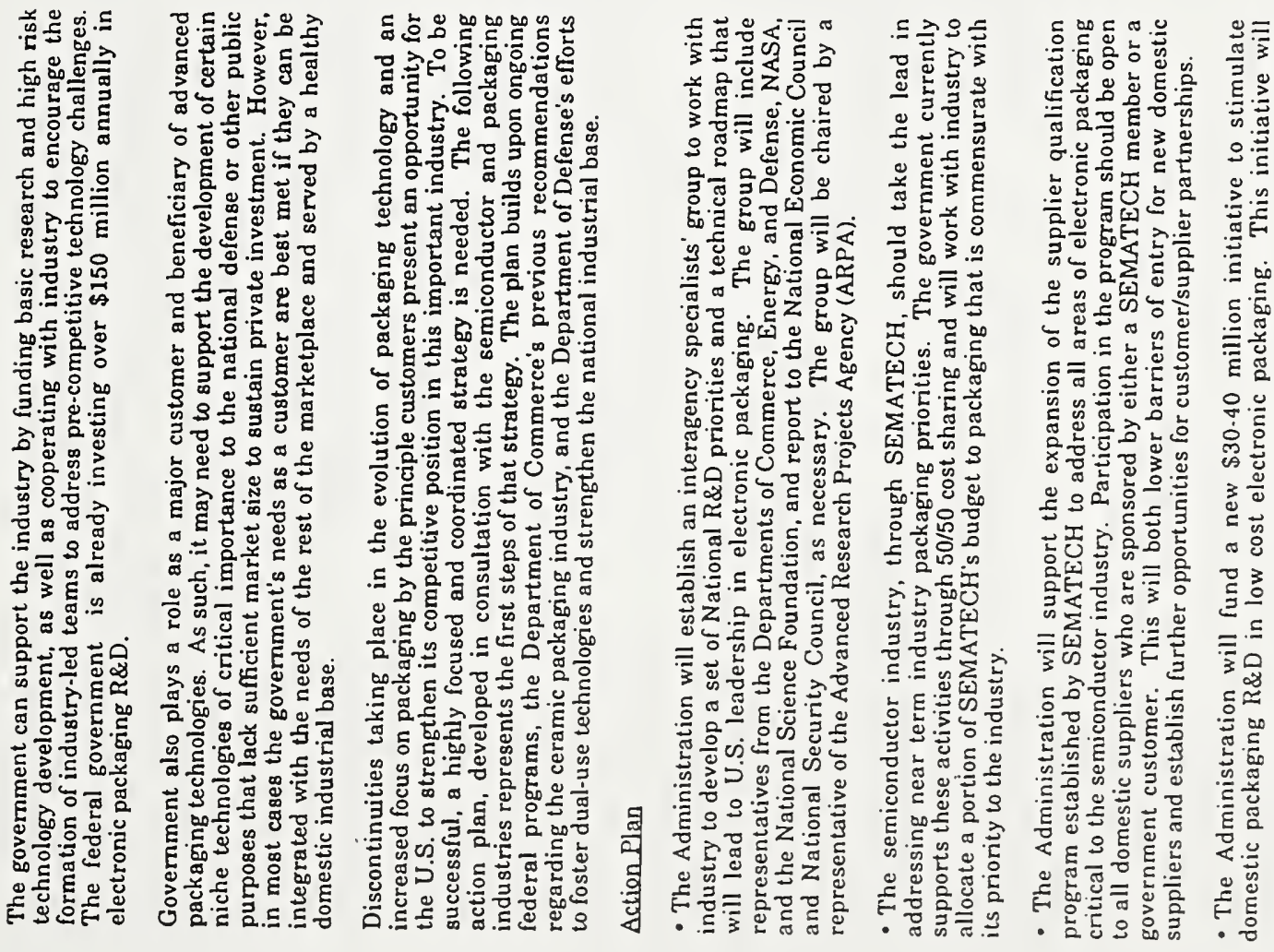



